\title{
Regularity of almost minimizing sets
}

\author{
Reinaldo Resende de Oliveira
}

DisSERTATION PRESENTED

$\mathrm{TO}$

Institute of Mathematics And Statistics

OF

University OF SÃo PAUlo

FOR

GETTING THE DEGREE

OF

MASTER IN SCIENCE

Program: Master in Mathematics

Advisor: Prof. Dr. Gláucio Terra

Coadvisor: Prof. Dr. Stefano Nardulli

During the development of this work the author received a scolarship of $\mathrm{CNPq}$

São Paulo, March of 2019 


\section{Regularity of almost minimizing sets}

This is the original version of the dissertation done by the candidate (Reinaldo Resende de Oliveira), as submitted to the Judging Committee. 


\section{Regularity of almost minimizing sets}

This version of the dissertation include the fixes and changes suggested

by the Judging Committee during the presentation of the original version of this work, performed on July 31, 2019. One copy of the original version is available

in Institute of Mathematics and Statistics of University of São Paulo.

Judging Committee:

- Prof. Dr. Gláucio Terra (advisor) - IME-USP

- Prof. Dr. Márcio Fabiano da Silva - UFABC

- Prof. Dr. Sinuê Dayan Barbero Lodovici - UFABC 


\section{Abstract}

Resende, R. O. Regularity of almost minimizing sets. 2019. 120 f. Thesis (Master) - Institute of Mathematics and Statistics, University of São Paulo, São Paulo, 2019.

This work was motivated by the famous Plateau's Problem which concerns the existence of a minimizing set of the area functional with prescribed boundary. In order to solve the Plateau's Problem, we make use of different theories: the theory of varifolds, currents and locally finite perimeter sets (Caccioppoli sets). Working on the Caccioppoli sets theory, it is straightforward to prove the existence of a minimizing set in some classical problems as the isoperimetric and Plateau's problems. If we switch the problem to find the regularity that we can extract of some minimizing set, we come across complicated ideas and tools. Although, the Plateau's Problem and other classical problems are well settled. Because of that, we have extensively studied the almost minimizing condition $((\lambda, r)$-minimizing sets) considered by Maggi ([Mag12]) which subsumes some classical problems. We focused on the regularity theory extracted from this almost minimizing condition.

Keywords: Caccioppoli, almost minimizing, minimizing, geometric measure theory, regularity theory, locally finite perimeter, finite perimeter. 


\section{Contents}

Symbols List $\quad$ v

0 Prologue 5

0.1 Functions of bounded variation . . . . . . . . 5

0.2 Caccioppoli sets . . . . . . . . . . . . 7

0.3 Hausdorff measure . . . . . . . . . . . . . . 8

0.4 The reduced boundary . . . . . . . . . . . 9

1 The almost minimizing sets $\quad 11$

1.1 Definition of the almost minimizing condition . . . . . 11

1.2 Basic properties of the almost minimizing sets . . . . . 12

1.3 Density estimates . . . . . . . . . . . . . . 14

1.4 Compactness theorems . . . . . . . . . . . . 18

2 Excess theory $\quad 27$

2.1 Excess and its basic properties . . . . . . . . . 27

2.2 Bounded excess consequences . . . . . . . . . 33

3 Approximation theorems $\quad 47$

3.1 Lipschitz boundary criterion . . . . . . . . . . 47

3.2 Lipschitzian approximation . . . . . . . . . 50

3.3 Approximations results on harmonic functions . . . . . 59

4 Regularity theory $\quad 63$

4.1 Reverse Poincaré inequality . . . . . . . . . . 63

4.2 Excess revisited . . . . . . . . . . . . 64

$4.3 C^{1, \gamma}$-regularity of the almost minimizing sets $\ldots \ldots . . .73$

$\begin{array}{ll}\text { Bibliography } & 81\end{array}$ 
iv CONTENTS 


\section{Symbols List}

$E^{c}=\mathbb{R}^{n} / E$

$\operatorname{int}(E)$ is the topological interior of $E$

$\bar{E}$ is the topological closure of $E$

$\partial E=\bar{E} \backslash \operatorname{int}(E)$

$E_{t}=\left\{z \in \mathbb{R}^{n-1}:(z, t) \in E\right\}$

$E \subset \mathbb{R}^{n}$ then $1_{E}(x)=\left\{\begin{array}{c}1, x \in E \\ 0, x \in E^{c}\end{array}\right.$

$G(u)$ denotes the graph of $u$

Lip $(u)$ is the Lipschitz constant of $u$

$\operatorname{supp} f=\overline{\{x \in \operatorname{dom}(f): f(x) \neq 0\}}$

For $U \subset \mathbb{R}^{n}$ open set $C^{k}\left(U, \mathbb{R}^{m}\right)=$

$=\left\{f: U \rightarrow \mathbb{R}^{m}\right.$ : the $i$-th derivate $f^{(i)}$ is continuous whenever $\left.0 \leq i \leq k\right\}$

For $U \subset \mathbb{R}^{n}$ open set $C_{c}^{k}\left(U, \mathbb{R}^{m}\right)=\left\{f \in C^{k}\left(U, \mathbb{R}^{m}\right): \operatorname{supp} f \Subset U\right\}$

$J f$ is the Jacobian of $f$

$f, g: X \rightarrow Y$ then $\{x \in X: f(x)=g(x)\}=\{f=g\}$

$\mathbf{B}(x, r)=\left\{y \in \mathbb{R}^{n}:|x-y|<r\right\}$

$\mathbb{D}_{r}=\left\{z \in \mathbb{R}^{n-1}:|z| \leq r\right\}$

$\mathbf{p}: \mathbb{R}^{n} \rightarrow \mathbb{R}^{n-1}$ is defined as $\mathbf{p}\left(x_{1}, \ldots, x_{n}\right)=\left(x_{1}, \ldots, x_{n-1}\right)$

$\mathbf{q}: \mathbb{R}^{n} \rightarrow \mathbb{R}$ is defined as $\mathbf{q}\left(x_{1}, \ldots, x_{n}\right)=x_{n}$

$u \cdot v=\sum_{i=1}^{n} u_{n} v_{n}$ where $u=\left(u_{1}, \ldots, u_{n}\right), v=\left(v_{1}, \ldots, v_{n}\right)$

$u, v, w \in \mathbb{R}^{n}$ then $(u \otimes v) w=(v \cdot w) u$

$\|z\|_{\nu} \doteq \max \left\{\left|\operatorname{proj}_{\nu} z\right|,\left|\operatorname{proj}_{\nu^{\perp}} z\right|\right\}$ where $\operatorname{proj}$ is the euclidean orthogonal projection

$\mathbf{C}(x, r, \nu)=\left\{y \in \mathbb{R}^{n}:\|y-x\|_{\nu}<r\right\}$

$\mu$ a measure, then $E \stackrel{\mu}{\sim} F \Leftrightarrow \mu(E \Delta F)=0$

$\mu$ a measure on $\mathbb{R}^{n}$, then $\mu\left\llcorner A_{A}\right.$ denotes the restriction to $A$

spt $\mu=\left\{x \in \mathbb{R}^{n}: \forall r>0, \mu(\mathbf{B}(x, r))>0\right\}$ is the support of the measure $\mu$

$\phi \sharp \mu$ is the push-forward of $\mu$ by $\phi$

$|\mu|$ is the total variation of the measure $\mu$

$|A|$ is the Lebesgue measure of $A$

$\omega_{n}=|\mathbf{B}(0,1)|$ is the volume of the ball $\mathbf{B}(0,1)$ 


\section{Introduction}

Geometric measure theory (GMT) has roots going back to ancient Greek mathematics. Ancient Greek mathematicians worked on the isoperimetric problem (to find the planar domain of given perimeter having greatest area) which leads naturally to questions about spatial regions and boundaries. The so-called Plateau's problem (to find the minimal surface with prescribed boundary), named in honor of the Belgian physicist Joseph Plateau, also belongs to the roots of GMT and has been studied since the 18th century. Despite its elderly roots, GMT has evolved, in the 20th century, into a modern theory which is in a confluence zone between Geometry and Mathematical Analysis. The name "geometric measure theory" owes its origins to Federer's masterpiece published in 1969 ([Fed96]).

A basic idea in GMT is to generalize the classic differential-geometric notion of surface in order to enlarge the set of possible solutions to Plateau's and similar variational problems ${ }^{1}$. According to the strategy used to generalize the notion of surface, the development of the theory has taken different paths, among which we may identify three main branches:

De Giorgi: The theory of Caccioppoli sets (or locally finite perimeter sets), named after the Italian mathematician Renato Caccioppoli, who introduced those sets in [Cac27]. In this formalism, a hypersurface is defined as the boundary of a Caccioppoli set. De Giorgi made important contributions to this theory, which can be found in [DG54], [DG55], [DG61a] and [DG61b]. We also refer the reader to H. Federer's book ([Fed96]) and more recent references, such as Lawrence C. Evans and Ronald F. Gariepy ([LCE92]), Leon Simon ([Sim83]), Luigi Ambrosio, Nicola Fusco and Diego Pallara ([LA00]), Fanghua Lin and Xiaoping Yang ([FL03]), Enrico Giusti ([Giu84]), Francesco Maggi ([Mag12]) and Frank Morgan ([Mor00]).

Federer and Fleming: The theory of normal and integral currents ([FF60], [Fed96]). Federer and Fleming defined a $k$-dimensional surface in $\mathbb{R}^{n}$ as a $k$-current, i.e. a continuous linear functional on the space of $k$-forms endowed with an inductive limit topology of Fréchet spaces. Those objects generalize the theory of Schwartz's distributions. This method is extensively studied in [Fed96] and it generalizes the theory of finite perimeter sets (which may be identified

\footnotetext{
${ }^{1}$ making it possible to apply the so-called direct method of the Calculus of Variations.
} 
with a particular type of normal currents) to codimensions bigger than 1.

Almgren and Allard: The theory of varifolds, which was introduced by Almgren ([ALM65]) and Allard ([All72], [All87]). They defined a $k$-dimensional varifold as a Radon measure on $\mathbb{R}^{n} \times G r(n, k)$ where $\operatorname{Gr}(n, k)$ denotes the Grassmann manifold of the $k$-hyperplanes in $\mathbb{R}^{n}$. They have also shown that the varifolds are a more general concept than that of currents.

Each one of the above methods is adequate to study variational problems such as the classical Plateau's problem and the isoperimetric problem. In this work, we will focus on the theory of Caccioppoli sets. In this framework, one can easily show the existence of minimal solutions to the aforementioned problems by means of the direct method of the calculus of variations. The study of the regularity of such solutions, however, is really tough.

There exist certain minimizers in geometric variational problems with volume-constraints, potential-type energies and the like, that do not satisfy the usual minimality condition, i.e. without constraints. In order to encompass such problems into the framework, it arises the interest to introduce a notion of almost minimality, i.e. a relaxed notion of minimality. There are many ways to introduce such a relaxed notion; one of them was originally presented on a highly general context by Almgren in 1974 ([Alm76]) aiming to solve elliptic variational problems with constraints. One year after Almgren's work, Massari introduced another notion of minimality with constraints in [Mas75]. Indeed, if we set $\mathcal{P}(E, \cdot)$ the perimeter measure of the Cacciopoli set $E$ and we define the functional $T_{g}$ in the class of Caccioppoli sets depending on $g \in L^{p}(\Omega), p>n, \Omega \subset \mathbb{R}^{n}$ by

$$
\mathbf{T}_{g}(E)=\mathcal{P}(E, K)+\int_{K \cap E} g(x) \mathrm{d} x
$$

where $K$ is a compact subset of $\Omega$, then $F$ will be a minimizer in Massari's concept ([Mas75]) if

$$
\mathbf{T}_{g}(F) \leq \mathbf{T}_{g}(E)
$$

for all $K$ compact subset of $\Omega$ and all Caccioppoli set $E$ such that $E \Delta F \subset$ $K$. Massari's minimizers also satisfies

$$
\mathcal{P}(F, K) \leq \mathcal{P}(E, K)+\|g\|_{p}|E \Delta F|^{\frac{p-1}{p}}
$$

Another almost minimality condition was introduced by Tamanini in 1984 ([Tam84]). Tamanini defined an almost minimizing set in $\Omega \subset \mathbb{R}^{n}$ as a Caccioppoli set $F$ such that for all $A \Subset \Omega$ exist $t \in(0, \operatorname{dist}(A, \partial \Omega)$ and $\alpha:(0, t) \rightarrow[0, \infty)$ non-decreasing function with $\lim _{r \rightarrow 0^{+}} \alpha(r)=0$ which satisfy

$$
\mathcal{P}(E, \mathbf{B}(x, r)) \leq \mathcal{P}(F, \mathbf{B}(x, r))+\alpha(r) r^{n-1}
$$

for every $x \in A, r \in(0, t)$ and $E$ Caccioppoli set with $E \Delta F \Subset \mathbf{B}(x, r)$. In this work, we will adopt the concept of almost minimality proposed in 
[Mag12]. Instead of consider either 0.1 or 0.2 , Maggi introduces a $(\Lambda, r)$ minimality condition, which we define rigorously in Definition 1.1.

The regularity problems which will be the main goal of this work are widely spread in the literature ([Giu84], [Mag12], [Alm76], [Mas75], [Sim83], [LA00], [Fed96], [Tam84], [DG61b]), with some minor variations on the definition of almost minimality adopted. In fact, regularity theorems can be proved under weaker minimality conditions than the $(\Lambda, r)$ minimality. For instance, Massari ([Mas75]) asserted that the minimizer of the functional defined in 0.1 has reduced boundary $\Omega \cap \partial^{*} F$ of class $C^{1, \frac{p-n}{4 p}}$ with the restriction on the dimension $p>n \geq 8$. On the other hand, Tamanini ([Tam84]) established that the minimizer of 0.2 has reduced boundary $\Omega \cap \partial^{*} F$ of class $C^{1}$ under some forced conditions on $\alpha$.

We aim to exploit the regularity that can be extracted from the class of minimizers defined in ([Mag12]). For this purpose, the notion of regularity will come up in many ways. The main tool that we will discuss is the excess which is used to measure the oscillation of the measuretheoretic outer unit normal of a Caccioppoli set. The precise definition of the excess can be found in Definition 2.1. The smallness of the excess allows us to collect technical results about the Caccioppoli set as the Height Bound (Theorem 2.17) and the Lipschitz Approximation (Theorem 3.3), which are the main tools to prove the regularity theorem that we will presented in this work. That is, the $C^{1, \gamma}$-regularity of the almost minimizing sets Theorem. 


\section{Prologue}

The propose of this introductory chapter is to fix some notation and terminology. Furthermore, we will also state some well known results on geometric measure theory which will be used in the deployment of the almost minimizing sets theory. For more basic notation, one can check the Symbols List (see page v).

\subsection{Functions of bounded variation}

Let $(U, \Sigma, \mu)$ be a measurable space, then we define the space of locally $p$-integrable functions $L_{l o c}^{p}(U, \mu), 1 \leq p<\infty$ as follows, if $f: U \rightarrow \mathbb{R}$ is measurable,

$$
f \in L_{l o c}^{p}(U, \mu) \Leftrightarrow\left(\int_{K}|f|^{p} \mathrm{~d} \mu\right)^{\frac{1}{p}}<\infty, \quad \forall K \Subset U
$$

If the right side on the inequality holds for $U$, i.e.

$$
\left(\int_{U}|f|^{p} \mathrm{~d} \mu\right)^{\frac{1}{p}}<\infty
$$

we write $f \in L^{p}(U, \mu)$ to denote the space of such functions. The space $L_{l o c}^{p}(U, \mu), 1 \leq p<\infty$ with the following norm, $f \in L_{l o c}^{p}(U, \mu)$,

$$
\|f\|_{p} \doteq\left(\int_{U}|f|^{p} \mathrm{~d} \mu\right)^{\frac{1}{p}}
$$

is a Banach Space. For the sake of brevity, we set $L_{l o c}^{p}(U,|\cdot|)=L_{l o c}^{p}(U)$ and $L^{p}(U,|\cdot|)=L^{p}(U)$ for any $U \subset \mathbb{R}^{n}$ where $|\cdot|$ denotes the Lebesgue measure on $\mathbb{R}^{n}$.

We call the functions in $L_{l o c}^{1}(U)$ (respectively, $L^{1}(U)$ ) by locally integrable 
functions (resp., integrable functions). We will use the notation, for all $A, B \subset U$,

$$
A \rightarrow B
$$

to describe that $1_{A} \rightarrow 1_{B}$ in $L^{1}(U)$.

If $U \subset \mathbb{R}^{n}$ denotes an open set, we say that $f \in L_{\text {loc }}^{1}(U)$ has locally bounded variation in $U$, if

$$
\sup \left\{\int_{K} f(x) \operatorname{div} \phi(x) \mathrm{d} x: \phi \in C_{c}^{1}\left(K, \mathbb{R}^{n}\right),|\phi| \leq 1\right\}<\infty
$$

for all $K \Subset U$. The space of the locally bounded variation functions is denoted by $B V_{l o c}(U)$. We also establish the notation $B V(U)$ to denote the space of bounded variation functions, i.e. $f \in L^{1}(U)$ such that

$$
\sup \left\{\int_{U} f(x) \operatorname{div} \phi(x) \mathrm{d} x: \phi \in C_{c}^{1}\left(U, \mathbb{R}^{n}\right),|\phi| \leq 1\right\}<\infty
$$

We recall the Structure Theorem for $B V_{l o c}$ (Theorem 5.1 in [LCE92]) which states, for each $f \in B V_{l o c}(U)$, the existence of a Radon measure $\mu_{f}$ on $U$ and a $\mu_{f}$-measurable function $\nu_{f}: U \rightarrow \mathbb{R}^{n}$ such that $\left|\nu_{f}(x)\right|=1$ $\mu_{f}$-almost everywhere $x \in U$ and

$$
\int_{U} f(x) \operatorname{div} \phi(x) \mathrm{d} x=-\int_{\mathbb{R}^{n}} \phi \cdot \nu_{f} \mathrm{~d} \mu_{f} \quad \forall \phi \in C_{c}^{1}\left(U, \mathbb{R}^{n}\right)
$$

For bounded variation functions, we have the Lower Semicontinuity Theorem (Theorem 5.2 in [LCE92]) which establish that, if $\left\{f_{i}\right\}_{i \in \mathbb{N}} \subset B V(U)$ and $f_{i} \rightarrow f$ in $L_{l o c}^{1}(U)$,

$$
\mu_{f}(U) \leq \liminf _{i \rightarrow \infty} \mu_{f_{i}}(U)
$$

If we consider the norm, $f \in B V(U)$,

$$
\|f\|_{B V(U)}=\|f\|_{1}+\mu_{f}(U)
$$

we find that $B V(U) \cap C^{\infty}(U)$ is a dense subspace of $B V(U)$. If we now consider $U$ open, bounded and with Lipschitz boundary, we can state a sort of compactness, i.e. if $\left\{f_{i}\right\} \subset B V(U)$ with

$$
\sup _{i}\left\|f_{i}\right\|_{B V(U)}<\infty
$$

we can take a subsequence $\left\{f_{i_{k}}\right\}$ and $f \in B V(U)$ such that $f_{i_{k}} \rightarrow f$ in $L^{1}(U)$ (Theorem 5.5 in [LCE92]). 


\subsection{Caccioppoli sets}

If $E, U \subset \mathbb{R}^{n}$ with $U$ an open set and $1_{E} \in B V_{l o c}(U)$ (resp., $B V(U)$ ), we say that $E$ is a set of locally finite perimeter (resp., finite perimeter) in $U$. Hereafter, we set

$$
\begin{gathered}
\nu_{1_{E}}=\nu_{E} \\
\mu_{1_{E}}(\cdot)=\mathcal{P}(E, \cdot) \\
\mu_{E}=\nu_{1_{E}} \mu_{1_{E}}
\end{gathered}
$$

where $\nu_{1_{E}}, \mu_{1_{E}}$ are given by the Structure Theorem for $B V_{l o c}(U)$ which we mentioned before. We notice that $\mu_{E}$ is a $\mathbb{R}^{n}$-valued Radon measure on $U$. The sets of locally finite perimeter are also called Caccioppoli sets in many books of Geometric Measure Theory. Accordingly, we will use this terminology. We call by perimeter measure the Radon measure $\mathcal{P}(E, \cdot)$ on $U$ and it holds that

$$
\mathcal{P}(E, \cdot)=\left|\mu_{E}\right|(\cdot)
$$

i.e. the perimeter measure is the total variation of the measure $\mu_{E}$. We notice that

$$
\mu_{E}=D 1_{E}
$$

where we denoted by $D 1_{E}$ the distributional derivative of $1_{E}$. Now, we shall prove a result that is simple calculation, but we have not seen its proof in the literature.

Proposition 0.1. Let $E$ be a Caccioppoli set in $\mathbb{R}^{n}$. If $F$ is equivalent to $E$, i.e. $|E \Delta F|=0$, it follows that

(i) $F$ is a Caccioppoli set;

(ii) $\mu_{F}=\mu_{E}$

Proof. (i) By the definition of Caccioppoli set, we have

$$
\sup \left\{\int_{E} \operatorname{div} T(x) \mathrm{d} x: T \in C_{c}^{1}\left(\mathbb{R}^{n}, \mathbb{R}^{n}\right), \operatorname{spt} T \subset K, \sup _{\mathbb{R}^{n}}|T| \leq 1\right\}<\infty
$$

for every $K \subset \mathbb{R}^{n}$ compact set. Since $F$ and $E$ are equivalent, we find

$$
\int_{E} \operatorname{div} T(x) \mathrm{d} x=\int_{F} \operatorname{div} T(x) \mathrm{d} x
$$

Then, for every $K \subset \mathbb{R}^{n}$ compact set, we conclude that

$$
\sup \left\{\int_{F} \operatorname{div} T(x) \mathrm{d} x: T \in C_{c}^{1}\left(\mathbb{R}^{n}, \mathbb{R}^{n}\right), \operatorname{spt} T \subset K, \sup _{\mathbb{R}^{n}}|T| \leq 1\right\}<\infty
$$

Therefore, $F$ is a Caccioppoli set.

(ii) Since $1_{F}$ and $1_{E}$ are equal as elements of $L_{l o c}^{1}$, we can ensure that the distributional derivatives $D 1_{E}$ and $D 1_{F}$ will also be equal and thus $\mu_{E}=\mu_{F}$. 
The converse of the last proposition is also true. However, we will not prove it here. The last proposition provides a type of invariance of $\mu_{E}$ and the perimeter measure under modifications on sets Lebesgue-null sets. Since for any Caccioppoli set $E$ in $\mathbb{R}^{n}$ we have

$$
\text { spt } \mu_{E}=\left\{x \in \mathbb{R}^{n}: 0<|E \cap \mathbf{B}(x, r)|<\omega_{n} r^{n}, \quad \forall r>0\right\} \subset \partial E
$$

we can show the existence of $F \stackrel{\text { Lesbegue }}{\sim} E$ such that

$$
\text { spt } \mu_{F}=\partial F
$$

and then $\mu_{F}=\mu_{E}$. If $E$ and $F$ are Caccioppoli sets in $\mathbb{R}^{n}$ (resp., finite perimeter), we have that $E \cap F$ and $E \cup F$ are also Caccioppoli sets in $\mathbb{R}^{n}$ (resp., finite perimeter) and it holds that

$$
\mathcal{P}(E \cup F, \cdot)+\mathcal{P}(E \cap F, \cdot) \leq \mathcal{P}(E, \cdot)+\mathcal{P}(F, \cdot)
$$

The isoperimetric inequality in the euclidean space $\mathbb{R}^{n \geq 2}$, i.e.

$$
n \omega^{\frac{1}{n}}|E|^{\frac{n-1}{n}} \leq \mathcal{P}\left(E, \mathbb{R}^{n}\right) \quad \forall E \subset \mathbb{R}^{n} \text { with }|E|<\infty
$$

is an indispensable tool in geometric measure theory. It can be also stated in balls (Proposition 12.37 in [Mag12]) for any Caccioppoli set $E$ in $\mathbb{R}^{n}$ such that

$$
|E \cap \mathbf{B}(x, r)| \leq t|\mathbf{B}(x, r)|
$$

whenever $n \geq 2, x \in \mathbb{R}^{n}, r>0, t \in(0,1)$. Indeed, under these assumptions on $E$, exists $c(n, t)$ depending only on $n$ and $t$ such that

$$
c(n, t)|E \cap \mathbf{B}(x, r)|^{\frac{n-1}{n}} \leq \mathcal{P}(E, \mathbf{B}(x, r))
$$

\subsection{Hausdorff measure}

We now set diam to be the diameter function on $\mathbb{R}^{n}$. For $k \in[0, \infty)$, the $k$-dimensional Hausdorff measure of $\Omega \subset \mathbb{R}^{n}$ is defined as follows

$$
\mathcal{H}_{k}(\Omega)=\lim _{\delta \rightarrow 0} \mathcal{H}_{k}^{\delta}(\Omega)
$$

where, for $\delta \in(0, \infty]$,

$$
\mathcal{H}_{k}^{\delta}(\Omega)=\frac{\omega_{k}}{2^{k}} \inf \left\{\sum_{i \in \mathbb{N}}\left(\operatorname{diam}\left(\Omega_{i}\right)\right)^{k}: \operatorname{diam}\left(\Omega_{i}\right)<\delta, \Omega \subset \bigcup_{i \in \mathbb{N}} \Omega_{i}\right\}
$$


The $k$-dimensional Hausdorff measure has some good properties, for instance, the behavior under translation and homotheties. Indeed, $\forall x \in$ $\mathbb{R}^{n}, \lambda>0$, we have

$$
\mathcal{H}_{k}(\lambda \Omega+x)=\lambda^{k} \mathcal{H}_{k}(\Omega) \quad \text { for any } \Omega \subset \mathbb{R}^{n}
$$

Since the $k$-dimensional Hausdorff measure is defined in function of the diameter, we can also ensure that

$$
\mathcal{H}_{k}(f(\Omega)) \leq(\operatorname{Lip}(f))^{k} \mathcal{H}_{k}(\Omega)
$$

for all Lipschitz function $f$ and $\Omega \subset \mathbb{R}^{n}$. One of the main results regarding the Hausdorff measure is that

$$
\mathcal{H}_{n}(\Omega)=|\Omega| \quad \forall \Omega \subset \mathbb{R}^{n} \text { Borel set }
$$

To finish this section, let us state the Coarea and Area formulas. If $f$ : $\mathbb{R}^{n} \rightarrow \mathbb{R}^{m}$ and $g: \mathbb{R}^{m} \rightarrow \mathbb{R}^{n}$ are Lipschitz functions, $n \geq m$, for each $A \subset \mathbb{R}^{n}, B \subset \mathbb{R}^{m}$ Lebesgue measurable sets, we have

(Coarea Formula) $\quad \int_{A} J f(x) \mathrm{d} x=\int_{\mathbb{R}^{m}} \mathcal{H}_{n-m}\left(A \cap f^{-1}(y)\right) \mathrm{d} y$

(Area Formula) $\quad \int_{B} J g(y) \mathrm{d} y=\int_{\mathbb{R}^{n}} \mathcal{H}_{0}\left(B \cap g^{-1}(x)\right) \mathrm{d} x$

\subsection{The reduced boundary}

The reduced boundary $\partial^{*} E$ of a Caccioppoli set $E$ is the set of points $x \in \mathbb{R}^{n}$ such that $x \in \operatorname{spt} \mu_{E}$ and

$$
\nu_{E}(x)=\lim _{r \rightarrow 0^{+}} \frac{\mu_{E}(\mathbf{B}(x, r))}{\mathcal{P}(E, \mathbf{B}(x, r))} \in \mathbb{S}^{n-1}
$$

The vector $\nu_{E}$ is called the measure-theoretic outer unit normal to E. We use 0.1 and the definition of reduced boundary to be able to consider sets with

$$
\overline{\partial^{*} E}=\operatorname{spt} \mu_{E}=\partial E
$$

One of the main theorems in geometric measure theory is the De Giorgi's Structure Theorem (Theorem 4.4 in [Giu84]) which asserts, for a Caccioppoli set $E$, that

$$
\mu_{E}=\nu_{E} \mathcal{H}_{n-1}\left\llcorner\partial^{*} E\right.
$$


and

$$
\mathcal{P}(E, \cdot)=\left|\mu_{E}\right|(\cdot)=\mathcal{H}_{n-1}\left(\partial^{*} E \cap \cdot\right)
$$

We define the points of density $t \in[0,1]$ of a set $E \subset \mathbb{R}^{n}$ as follows

$$
E^{(t)}=\left\{x \in \mathbb{R}^{n}: \lim _{t \rightarrow 0^{+}} \frac{|E \cap \mathbf{B}(x, r)|}{\omega_{n} r^{n}}=t\right\}
$$

Now, we recall the definition of the measure theoretic boundary of a set $E \subset \mathbb{R}^{n}$, i.e.

$$
\partial^{e} E=\mathbb{R}^{n} \backslash\left(E^{(1)} \cup E^{(0)}\right)
$$

For any Caccioppoli set $E \subset \mathbb{R}^{n}$, the well known Federer's Theorem states that

$$
\partial^{*} E \subset E^{(1 / 2)} \subset \partial^{e} E
$$

Moreover

$$
\mathcal{H}_{n-1}\left(\partial^{e} E \backslash \partial^{*} E\right)=0
$$

which can be found on Section 5.8 in [LCE92]. 


\section{The almost minimizing sets}

\subsection{Definition of the almost minimizing con- dition}

Let us precisely state the almost minimizing condition that we will consider in this work.

Definition 1.1. Let $A \subset \mathbb{R}^{n}$ be open and $E$ is a Caccioppoli set (or locally finite perimeter set) in $\mathbb{R}^{n}$. Then $E$ is called a $(\Lambda, r)$-minimizing in $A$ if spt $\mu_{E}=\partial E, \Lambda \geq 0, r>0$ and for all Caccioppoli set $F$ such that $E \Delta F \Subset A \cap \boldsymbol{B}(x, s), s<r, x \in A$ it holds

$$
\mathcal{P}(E, \boldsymbol{B}(x, s)) \leq \mathcal{P}(F, \boldsymbol{B}(x, s))+\Lambda|E \Delta F|
$$

$r$ is also called the scale and $F$ is called competitor.

Note that the condition spt $\mu_{E}=\partial E$ is not restrictive, since we can choose an $\mathcal{H}_{n}$-equivalent set with this property (by Proposition 12.19 in [Mag12]).

In the definition it holds $|E \Delta F| \leq \omega_{n} s^{n}$, i.e. the "error" factor is always bounded above by the volume of the balls where the competitors are different from $E$ and it decreases faster than the scale. Recalling the De Giorgi's structure theorem (Theorem 4.4 in [Giu84]) which states $\mathcal{P}(E, \cdot)=\mathcal{H}_{n-1}\left(\partial^{*} E \cap \cdot\right)$, it makes sense to keep in mind that the "error" factor behaves like a higher order perturbation.

Before the examples, let us explain why it will henceforth be considered the hypothesis $\Lambda r \leq 1$ in this work. For this purpose, suppose that $E$ is a $(\Lambda, r)$ - minimizing set in $A$ and $F$ is a competitor, it follows from the euclidean isoperimetric inequality that

$$
\begin{gathered}
|E \Delta F|=|E \Delta F|^{\frac{1}{n}}|E \Delta F|^{\frac{n-1}{n}} \leq\left(\omega_{n} s^{n}\right)^{\frac{1}{n}} \frac{\mathcal{P}\left(E \Delta F, \mathbb{R}^{n}\right)}{n \omega_{n}^{\frac{1}{n}}}={ }^{*} \\
={ }^{*} \frac{s}{n} \mathcal{P}(E \Delta F, \mathbf{B}(x, s)) \leq^{* *} \frac{s}{n}(\mathcal{P}(F, \mathbf{B}(x, s))+\mathcal{P}(E, \mathbf{B}(x, s)))
\end{gathered}
$$

where $(*)$ follows from $E \Delta F \Subset \mathbf{B}(x, s)$ and $(* *)$ is consequence of the perimeter property $\left(E_{1}, E_{2}\right.$ being Caccioppoli sets, then $\mathcal{P}\left(E_{1} \cup E_{2}, \cdot\right) \leq$ 
$\left.\mathcal{P}\left(E_{1}, \cdot\right)+\mathcal{P}\left(E_{2}, \cdot\right)\right), \mathcal{P}(E, \cdot)=\mathcal{H}_{n-1}\left(\partial^{*} E \cap \cdot\right)=\mathcal{H}_{n-1}\left(\partial^{e} E \cap \cdot\right)$ and $\partial^{e}(E \Delta F) \subset \partial^{e} E \cup \partial^{e} F$. Therefore

$$
\begin{gathered}
\mathcal{P}(E, \mathbf{B}(x, s)) \leq \mathcal{P}(F, \mathbf{B}(x, s))+\Lambda|E \Delta F| \leq \\
\leq \mathcal{P}(F, \mathbf{B}(x, s))+\Lambda \frac{s}{n}(\mathcal{P}(E, \mathbf{B}(x, s))+\mathcal{P}(F, \mathbf{B}(x, s)))
\end{gathered}
$$

It follows

$$
\left(1-\frac{\Lambda s}{n}\right) \mathcal{P}(E, \mathbf{B}(x, s)) \leq\left(1+\frac{\Lambda s}{n}\right) \mathcal{P}(F, \mathbf{B}(x, s))
$$

which is clearly trivial if $n \leq \Lambda s$, i.e. when the scale " $s$ " é too large. In the non-trivial case, $\Lambda s<n$, the scale $s$ is bounded, in general, we will fix "1" as the upper bound for $\Lambda s$ intending to help with some proofs ahead.

\subsection{Basic properties of the almost minimiz- ing sets}

Proposition 1.2. Let $E$ be a $(\Lambda, r)$ - minimizing set in $A$, then $E^{c}$ is $a(\Lambda, r)-$ minimizing set in $A$.

Proof. Since $1_{E}+1_{E^{c}} \equiv 1, E^{c}$ is also a Caccioppoli set and the distributional derivatives satisfies

$$
D 1_{E}=-D 1_{E^{c}} \Rightarrow \mu_{E}=-\mu_{E^{c}}
$$

Thus

$$
\operatorname{spt} \mu_{E^{c}}=\operatorname{spt} \mu_{E}=\partial E=\partial\left(E^{c}\right)
$$

Now, we take $F$ a competitor to $E^{c}$, i.e. $E^{c} \Delta F \Subset A \cap \mathbf{B}(x, s), s<r, x \in$ $A$, by the definition of the perimeter measure of $E$, i.e. $\mathcal{P}(E, \cdot)=\left|D 1_{E}\right|(\cdot)$, we find that

$$
\mathcal{P}\left(E^{c}, \cdot\right)=\mathcal{P}(E, \cdot)
$$

It is straightforward to verify that

$$
E^{c} \Delta F=E \Delta F^{c}
$$

what ensures that $F^{c}$ is a competitor to the almost minimality of $E$. 
Therefore, we have that

$$
\mathcal{P}\left(E^{c}, \mathbf{B}(x, s)\right)=\mathcal{P}(E, \mathbf{B}(x, s)) \leq \mathcal{P}\left(F^{c}, \mathbf{B}(x, s)\right)+\Lambda\left|E \Delta F^{c}\right|
$$

Since 1.3 is valid for $F$ in place of $E$, the last inequality becomes

$$
\mathcal{P}\left(E^{c}, \mathbf{B}(x, s)\right) \leq \mathcal{P}(F, \mathbf{B}(x, s))+\Lambda\left|E^{c} \Delta F\right|
$$

Let us define the blow-up of a arbitrary set $X \subset \mathbb{R}^{n}$ at $x$ at scale $r>0$ as follows

$$
E_{x, r}=\frac{E-x}{r}
$$

Now, we aim to prove the compatibility of the blow-up with the almost minimality condition.

Proposition 1.3. Given $y \in \mathbb{R}^{n}, r_{0}>0$. If $E$ is a $(\Lambda, r)$ - minimizing set in $A$, we have that $E_{y, r_{0}}$ is a $\left(\Lambda r, \frac{r}{r_{0}}\right)-$ minimizing set in $A_{y, r_{0}}$.

Remark 1.4. If we consider $T: \mathbb{R}^{n} \rightarrow \mathbb{R}^{n}$ a linear isometry, we have that (Proposition 2.51 in [VOL])

$$
\mu_{T(E)}=T \sharp \mu_{E} \quad\left|\mu_{T(E)}\right|=T \sharp\left|\mu_{E}\right|
$$

We call attention to the argument of this proof which can be easily adjusted to ensures that, if $E$ is a $(\Lambda, r)$-minimizing set in $A$, then $T(E)$ is a $(\Lambda, r)-$ minimizing set in $T(A)$.

Proof. Take $F$ a Caccioppoli set with $E_{y, r_{0}} \Delta F \subset A_{y, r_{0}} \cap \mathbf{B}(x, s), x \in$ $A_{y, r_{0}}, s<\frac{r}{r_{0}}$. We set

$$
\phi(z)=r_{0} z+y \quad \forall z \in \mathbb{R}^{n}
$$

Clearly, $\phi$ is a diffeomorphism,

$$
\phi(\mathbf{B}(x, s))=r_{0} \mathbf{B}(x, s)+y
$$

and

$$
\phi\left(E_{y, r_{0}}\right)=r_{0} E_{y, r_{0}}+y=E
$$

Analogously, we prove

$$
\phi\left(A_{y, r_{0}}\right)=A
$$

Taking into account $E_{y, r_{0}} \Delta F \subset A_{y, r_{0}} \cap \mathbf{B}(x, s)$, we find that

$$
\phi\left(E_{y, r_{0}}\right) \Delta \phi(F) \Subset \phi\left(A_{y, r_{0}}\right) \cap \phi(\mathbf{B}(x, s))
$$

Then, by 1.5, 1.6 and 1.7,

$$
E \Delta \phi(F) \Subset A \cap\left(r_{0} \mathbf{B}(x, s)+y\right)=A \cap \mathbf{B}\left(\phi(x), r_{0} s\right)
$$

Since $x \in A_{y, r_{0}}$ and $s<\frac{r}{r_{0}}$, we have $\phi(x) \in A$ and $r_{0} s<r$. Therefore, 
$\phi(F)$ is a competitor to $E$, then

$$
\mathcal{P}(E, \phi(\mathbf{B}(x, s))) \leq \mathcal{P}(\phi(F), \phi(\mathbf{B}(x, s)))+\Lambda|E \Delta \phi(F)|
$$

We intend to turn 1.8 into 1.1 for $E_{y, r_{0}}$. To this end, since $\phi$ is a diffeomorphism and $|J \phi| \equiv r_{0}^{n}$, we ensure that

$$
\begin{array}{r}
|E \Delta \phi(F)|={ }^{1.6}\left|\phi\left(E_{y, r_{0}} \Delta F\right)\right|= \\
=r_{0}^{n}\left|E_{y, r_{0}} \Delta F\right|
\end{array}
$$

Now, we focus on the term $\mathcal{P}\left(E, r_{0} \mathbf{B}(x, s)+y\right)$. Let us recall that

$$
\frac{\partial^{*} E-y}{r_{0}}=\partial^{*}\left(\frac{E-y}{r_{0}}\right)=\partial^{*}\left(E_{y, r_{0}}\right)
$$

By the nice properties of the Hausdorff measure under translations and hometheties (Proposition 2.49 in [LA00]), the De Giorgi's Structure Theorem and the last inequality, we obtain that

$$
\begin{array}{r}
\mathcal{P}(E, \phi(\mathbf{B}(x, s)))={ }^{1.5}=\mathcal{P}\left(E, r_{0} \mathbf{B}(x, s)+y\right)= \\
\mathcal{H}_{n-1}\left(\partial^{*} E \cap\left(r_{0} \mathbf{B}(x, s)+y\right)\right)=r_{0}^{n-1} \mathcal{H}_{n-1}\left(\left(\frac{\partial^{*} E-y}{r_{0}}\right) \cap \mathbf{B}(x, s)\right)= \\
r_{0}^{n-1} \mathcal{H}_{n-1}\left(\partial^{*}\left(E_{y, r_{0}}\right) \cap \mathbf{B}(x, s)\right)=r_{0}^{n-1} \mathcal{P}\left(E_{y, r_{0}}, \mathbf{B}(x, s)\right)
\end{array}
$$

We can apply the same argument to find that

$$
\mathcal{P}(\phi(F), \phi(\mathbf{B}(x, s)))=r_{0}^{n-1} \mathcal{P}(F, \mathbf{B}(x, s))
$$

By the last equality, 1.10 and 1.9, we can turn 1.8 into

$$
r_{0}^{n-1} \mathcal{P}\left(E_{y, r_{0}}, \mathbf{B}(x, s)\right) \leq r_{0}^{n-1} \mathcal{P}(F, \mathbf{B}(x, s))+r_{0}^{n}\left|E_{y, r_{0}} \Delta F\right|
$$

that is

$$
\mathcal{P}\left(E_{y, r_{0}}, \mathbf{B}(x, s)\right) \leq \mathcal{P}(F, \mathbf{B}(x, s))+\Lambda r_{0}\left|E_{y, r_{0}} \Delta F\right|
$$

what conclude the proof.

\subsection{Density estimates}

Now, we will start to progress with the theory and some meaningful theorems on the theory of the almost minimizing sets. One of the main 
theorems is the Density Estimates which makes it possible to prove both the compactness and closure theorem and other further results as well. The density estimates, by their own, put us in position to extract some geometric information about the almost minimizing sets. Indeed, the estimates show that the almost minimizing sets, and its boundary, have a quite good behavior whereas it is not obvious.

Theorem 1.5. (Density estimates) Let $n \geq 2$, exists $c(n)$ positive constant such that for all set $E$ which is a $\left(\Lambda, r_{0}\right)$ - minimizing in $A$ with $\Lambda r_{0} \leq 1$, it follows

$$
\begin{aligned}
& \frac{1}{4^{n}} \leq \frac{|E \cap \boldsymbol{B}(x, r)|}{\omega_{n} r^{n}} \leq 1-\frac{1}{4^{n}} \\
& c(n) \leq \frac{\mathcal{P}(E, \boldsymbol{B}(x, r))}{r^{n-1}} \leq 3 n \omega_{n}
\end{aligned}
$$

whenever $x \in A \cap \partial E, \boldsymbol{B}(x, r) \subset A$ and $r<r_{0}$.

Remark 1.6. The upper inequality in 1.12 is true for all $x \in A$, i.e. the restriction $x \in \partial E$ is not necessary. we intend to make this clear within the proof.

Proof. The upper estimate in 1.11 is a consequence of the lower estimate in 1.11 , because $E^{c}$ also is $\left(\Lambda, r_{0}\right)-$ minimizing in $A$ (by Proposition $1.2)$, thus

$$
\frac{1}{4^{n}} \leq \frac{\left|E^{c} \cap \mathbf{B}(x, r)\right|}{\omega_{n} r^{n}}=1-\frac{|E \cap \mathbf{B}(x, r)|}{\omega_{n} r^{n}}
$$

From the estimates in 1.11 and the relative isoperimetric inequality (Proposition 12.37 in [Mag12] putting $t=1-\frac{1}{4^{n}}$ ) that is

$$
\mathcal{P}(E, \mathbf{B}(x, r)) \geq c_{1}(n)|E \cap \mathbf{B}(x, r)|^{\frac{n-1}{n}}
$$

we can prove the lower estimate in 1.12 as follow

$$
\begin{gathered}
\frac{\mathcal{P}(E, \mathbf{B}(x, r))}{r^{n-1}} \geq \frac{c_{1}(n)|E \cap \mathbf{B}(x, r)|^{\frac{n-1}{n}}}{r^{n-1}} \geq \frac{c_{1}(n)}{r^{n-1}}\left(\frac{\omega_{n} r^{n}}{4^{n}}\right)^{\frac{n-1}{n}}= \\
=\frac{c_{1}(n) \omega_{n}^{\frac{n-1}{n}}}{4^{n-1}} \doteq c(n)
\end{gathered}
$$

Therefore, it is sufficient to proof the other two inequalities. For this purpose, fix $x \in A$, define $d=\min \left\{r_{0}\right.$, dist $\left.(x, \partial A)\right\}$ and $m(r)=\mid E \cap$ $\mathbf{B}(x, r) \mid, \forall r \in(0, d)$, then

$$
\begin{aligned}
m(r)=|E \cap \mathbf{B}(x, r)| & ={ }^{*} \int_{0}^{r} \mathcal{H}_{n-1}(E \cap \partial \mathbf{B}(x, t)) \mathrm{d} t \Rightarrow \\
& \Rightarrow m^{\prime}(r)=\mathcal{H}_{n-1}(E \cap \partial \mathbf{B}(x, r))
\end{aligned}
$$

for almost all $r \in(0, d)$, where the equality $(*)$ is a consequence of the Coarea formula (Theorem 3.12 in [LCE92]). Since $\mu_{E}=\mathcal{H}_{n-1{ }^{\circ} \partial^{*} E}$ is a 
Radon measure, it follows that, for almost all $r \in(0, d)$,

$$
\mathcal{H}_{n-1}\left(\partial^{*} E \cap \partial \mathbf{B}(x, r)\right)=0
$$

Being $r$ taken satisfying this equality, we aim to create a competitor of $E$ to use the $\left(\Lambda, r_{0}\right)$-minimality of $E$. Let $F=E \backslash \mathbf{B}(x, r)$. Clearly $E \Delta F \Subset \mathbf{B}(x, s) \Subset A, \forall s \in(r, d)$, thus, by 1.2 ,

$$
\left(1-\frac{\Lambda r}{n}\right) \mathcal{P}(E, \mathbf{B}(x, s)) \leq\left(1+\frac{\Lambda r}{n}\right) \mathcal{P}(F, \mathbf{B}(x, s))
$$

Moreover, from the operations with Caccioppoli sets (Theorem 16.3 in [Mag12]) and from the choice of $r$, it follows

$$
\begin{aligned}
\mathcal{P}(F & \mathbf{B}(x, s))=\mathcal{P}\left(E \cap(\mathbf{B}(x, r))^{c}, \mathbf{B}(x, s)\right)=\overbrace{\mathcal{P}\left(E,\left(\mathbf{B}(x, r)^{c}\right)^{(1)} \cap \mathbf{B}(x, s)\right)}^{*}+ \\
& +\overbrace{\mathcal{P}\left(\mathbf{B}(x, r), E^{(1)} \cap \mathbf{B}(x, s)\right)}^{* *}+\overbrace{\mathcal{H}_{n-1}\left(\left\{\mu_{E}=\mu_{\mathbf{B}(x, r)}\right\} \cap \mathbf{B}(x, s)\right)}^{=0}
\end{aligned}
$$

Note that in $(*)$, we have $\mathbf{B}(x, s) \cap\left(\mathbf{B}(x, r)^{c}\right)^{(1)} \subset \mathbf{B}(x, s) \backslash \overline{\mathbf{B}(x, r)}$, and in $(* *)$ apply the De Giorgi's structure theorem to come up with

$$
\mathcal{P}(F, \mathbf{B}(x, s)) \leq \mathcal{H}_{n-1}\left(E^{(1)} \cap \partial \mathbf{B}(x, r)\right)+\mathcal{P}(E, \mathbf{B}(x, s) \backslash \overline{\mathbf{B}(x, r)})
$$

Taking $s \rightarrow r^{+}$, it follows from 1.14 and 1.15 that

$$
\left(1-\frac{\Lambda r}{n}\right) \mathcal{P}(E, \mathbf{B}(x, r)) \leq\left(1+\frac{\Lambda r}{n}\right) \mathcal{H}_{n-1}\left(E^{(1)} \cap \partial \mathbf{B}(x, r)\right)
$$

Using that $\mathcal{H}_{n-1}\left(E^{(1)} \cap \partial \mathbf{B}(x, r)\right) \leq \mathcal{H}_{n-1}(\partial \mathbf{B}(x, r))=n \omega_{n} r^{n-1}$, and since $\frac{1+\frac{\Lambda r}{n}}{1-\frac{\Lambda r}{n}} \leq 3$, by $n \geq 2$ and $\Lambda r_{0} \leq 1$, the upper inequality in 1.12 is done for almost all $r \in(0, d)$. So, given $r \in(0, d)$, we choose a increasing sequence $\left\{r_{i}\right\}_{i \in \mathbb{N}}$ such that $r_{i} \rightarrow r$ and the upper inequality in 1.12 holds true for $r_{i}, \forall i \in \mathbb{N}$. By the continuity from below of the measure $\mathcal{P}(E, \cdot)$ (Theorem 1.8 in [Fol99]) and the continuity of $r^{n-1}$, we obtain that

$$
\begin{aligned}
\frac{\mathcal{P}(E, \mathbf{B}(x, r))}{r^{n-1}}= & \frac{\mathcal{P}\left(E, \cup_{i \in \mathbb{N}} \mathbf{B}\left(x, r_{i}\right)\right)}{r^{n-1}}=\frac{\lim _{i \rightarrow \infty} \mathcal{P}\left(E, \mathbf{B}\left(x, r_{i}\right)\right)}{\lim _{i \rightarrow \infty} r_{i}^{n-1}}= \\
& =\lim _{i \rightarrow \infty} \frac{\mathcal{P}\left(E, \mathbf{B}\left(x, r_{i}\right)\right)}{r_{i}^{n-1}} \leq 3 n \omega_{n}
\end{aligned}
$$

Then, the upper inequality in 1.12 is validated for all $r \in(0, d)$ and, along with this proof, we have only used that $x \in A$. Thus, the Remark 1.6 is also verified. Finally, we will prove the lower inequality in 1.11. To this end, suppose that $x \in A \cap \partial E$, then the function $m$ also satisfies

$$
0<m(r)<\omega_{n} r^{n}, \forall r \in(0, d)
$$

because $x \in \partial E=\operatorname{spt} \mu_{E}$. Adding $\left(1-\frac{\Lambda r}{n}\right) \mathcal{H}_{n-1}\left(E^{(1)} \cap \partial \mathbf{B}(x, r)\right)$ to both sides of 1.16 and using the operations with Caccioppoli sets (The- 
orem 16.3 in [Mag12], we obtain

$$
\left(1-\frac{\Lambda r}{n}\right) \mathcal{P}\left(E \cap \mathbf{B}(x, r), \mathbb{R}^{n}\right) \leq 2 \mathcal{H}_{n-1}\left(E^{(1)} \cap \partial \mathbf{B}(x, r)\right)
$$

By the isoperimetric inequality $\mathcal{P}\left(E \cap \mathbf{B}(x, r), \mathbb{R}^{n}\right) \geq n \omega_{n}^{\frac{1}{n}}|E \cap \mathbf{B}(x, r)|^{\frac{n-1}{n}}$, since $1-\frac{\Lambda r}{n} \leq 2$, from 1.13 and 1.17 , it follows that

$$
\frac{n \omega_{n}^{\frac{1}{n}}}{4} \leq \frac{m^{\prime}(r)}{(m(r))^{1-\frac{1}{n}}}
$$

Integrating 1.19 in the interval $(0, r)$, we get that (by Theorem 4.14 in [Gor94]) $\int_{0}^{r} \frac{m^{\prime}(t)}{(m(t))^{1-\frac{1}{n}}} \mathrm{~d} t=\int_{0}^{r} \frac{\mathrm{d}}{\mathrm{d} t}(n m(t))^{\frac{1}{n}} \mathrm{~d} t=n m(r)^{\frac{1}{n}}$, it follows

$$
\frac{r n \omega_{n}^{\frac{1}{n}}}{4} \leq n m(r)^{\frac{1}{n}}=n|E \cap \mathbf{B}(x, r)|^{\frac{1}{n}}
$$

Putting up the power of $n$ the proof is done.

We recall the definition of the essential boundary of a Caccioppoli set $E$, also called measure theoretic boundary, as follows

$$
x \in \partial^{e} E \Leftrightarrow x \in \mathbb{R}^{n} \backslash\left(E^{(1)} \cup E^{(0)}\right)
$$

The following corollary is a stronger version of the well known Federer's Theorem (Lemma 5.5 in [LCE92]) in the context of almost minimizing sets. The Federer's Theorem guarantees that $\partial^{e} E$ and $\partial^{*} E$ are $\mathcal{H}_{n-1^{-}}$ equivalent for any Caccioppoli set $E$. For the almost minimizing sets this result can be refined, ensuring the equivalence between the reduced boundary and the topological boundary. Here it is possible to conclude that the almost minimizing sets possess a kind of extra regularity, i.e. $\mathcal{H}_{n-1}$-almost everywhere it is possible to define a normal vector to $\partial E$.

Corollary 1.7. Let $n \geq 2$ and $E$ be $a\left(\Lambda, r_{0}\right)-$ minimizing set in $A$ with $\Lambda r_{0} \leq 1$. Then

$$
\mathcal{H}_{n-1}\left(A \cap\left(\partial E \backslash \partial^{*} E\right)\right)=0
$$

Proof. It is sufficient to note that 1.11 ensures that $\forall x \in A \cap \partial E, \forall r<r_{0}$

$$
\frac{1}{4^{n}} \leq \frac{|E \cap \mathbf{B}(x, r)|}{\omega_{n} r^{n}} \leq 1-\frac{1}{4^{n}}
$$

Thus, $0<\limsup _{r \rightarrow 0^{+}} \frac{|E \cap \mathbf{B}(x, r)|}{\omega_{n} r^{n}}<1$. By definition of essential boundary, we get that $x \in A \cap \partial^{e} E$. Therefore, $A \cap \partial E \subset A \cap \partial^{e} E$. Then, since $\partial^{*} E \subset \partial E$, the Federer's Theorem concludes the proof. 
Corollary 1.8. Let $n \geq 2$ and $E$ be $a\left(\Lambda, r_{0}\right)-$ minimizing set in $A$, with $\Lambda r_{0} \leq 1$, then

$$
c(n) \leq \frac{\mathcal{P}(E, C(x, r, \nu))}{r^{n-1}} \leq \sqrt{2}^{n-1} 3 n \omega_{n}
$$

whenever $x \in A \cap \partial E, \sqrt{2} r<r_{0}, \boldsymbol{B}(x, \sqrt{2} r) \subset A$.

Proof. It follows directly from 1.12, using that $\mathbf{B}(x, r) \subset \mathbf{C}(x, r, \nu) \subset$ B $(x, \sqrt{2} r)$.

\subsection{Compactness theorems}

In the excess theory and the approximation theorems, some constructions with sequences of almost minimizing sets will naturally appear for us. Hence, we shall work on it. For this purpose, we will extract a kind of compactness for the space of almost minimizing sets. As a consequence of the compactness of the sets of finite perimeter and the density estimates, the first result will ensure that for a sequence of almost minimizing sets, under some assumptions, we can find a set of finite perimeter which will be the limit set of the sequence.

Theorem 1.9. (Pre-compactness of the space of the almost minimizing sets) Let $n \geq 2$ and $\left(E_{h}\right)_{h \in \mathbb{N}}$ be a sequence such that each $E_{h}$ is $\left(\Lambda_{h}, r_{h}\right)$ - minimizing in $A$ with $\Lambda_{h} r_{h} \leq 1$ and $\liminf _{h \rightarrow \infty} r_{h}>0$. Then $\forall A_{0} \Subset A$ open set with finite perimeter, exists a set with finite perimeter $E \subset A_{0}$ and a subsequence $\left(E_{h^{\prime}}\right)_{h^{\prime} \in \mathbb{N}}$ such that

$$
A_{0} \cap E_{h^{\prime}} \rightarrow E \quad \text { and } \quad \mu_{A_{0} \cap E_{h^{\prime}}} \stackrel{*}{\rightarrow} \mu_{E}
$$

Proof. Given $A_{0} \Subset A$ open with finite perimeter, fix $x \in A_{0}$ and consider $\mathbf{B}(x, r) \Subset A$ and $0<r<\liminf _{h \in \mathbb{N}} r_{h}$ such that $\mathcal{H}_{n-1}\left(\partial^{*} E_{h} \cap \partial \mathbf{B}(x, r)\right)=$ 0 , that is possible, since $\mathcal{H}_{n-1}\left\llcorner\partial^{*} E_{h}\right.$ is Radon. The operations with Caccioppoli sets (Theorem 16.3 in [Mag12]) ensures the following equality

$$
\begin{gathered}
\mathcal{P}\left(E_{h} \cap \mathbf{B}(x, r), \mathbb{R}^{n}\right)= \\
=\mathcal{P}\left(E_{h}, \mathbf{B}(x, r)^{(1)}\right)+\mathcal{P}\left(\mathbf{B}(x, r), E_{h}^{(1)}\right)+\mathcal{H}_{n-1}\left(\left\{\nu_{E_{h}}=\nu_{\mathbf{B}(x, r)}\right\}\right) \leq \\
\leq^{*} \mathcal{P}\left(E_{h}, \mathbf{B}(x, r)\right)+\mathcal{P}\left(\mathbf{B}(x, r), \mathbb{R}^{n}\right) \leq^{* *} 3 n \omega_{n} r^{n-1}+n \omega_{n} r^{n-1}
\end{gathered}
$$

where $(*)$ follows from $\mathbf{B}(x, r)^{(1)}=\mathbf{B}(x, r), E_{h}^{(1)} \subset \mathbb{R}^{n}$ and

$$
\mathcal{H}_{n-1}\left(\left\{\nu_{E_{h}}=\nu_{\mathbf{B}(x, r)}\right\}\right) \leq \mathcal{H}_{n-1}\left(\partial^{*} E_{h} \cap \partial^{*} \mathbf{B}(x, r)\right)=0
$$


while, in order to apply the density estimates (1.12) in (**) and Remark 1.6, we assume that $h$ is sufficiently large, i.e. we fixed a $M \in \mathbb{N}$ such that $r<\inf _{h \geq M} r_{h}$. In short, if $x \in \partial E \cap A_{0}, r<\liminf _{h \rightarrow \infty} r_{h}$, it follows

$$
\sup _{h \geq M} \mathcal{P}\left(E_{h} \cap \mathbf{B}(x, r), \mathbb{R}^{n}\right)<4 n \omega_{n} r^{n-1}<\infty
$$

Since $A_{0}$ has compact closure in $A$, we can choose a finite family

$$
\left\{B_{j}=\mathbf{B}\left(x_{j}, r_{j}\right)\right\}_{j=1}^{N}
$$

which covers $A_{0}$ with $B_{j} \Subset A, x_{j} \in A_{0}$ and $r_{j}<\liminf _{h \rightarrow \infty} r_{h}$. From 1.21 and $E_{h} \cap B_{j} \subset B_{j}$, we are able to use the theorem of compactness for finite perimeter sets (Theorem 12.26 in [Mag12]), for each $j$, then, applying for $j=1$, exists $F_{1} \subset B_{1}$ finite perimeter set and $E_{h_{1}}$ subsequence such that

$$
B_{1} \cap E_{h_{1}} \rightarrow F_{1}
$$

Now, applying for the new subsequence $E_{h_{1}}$, with $j=2$, we got another subsequence. Using this idea successively until $j=N$, we have obtained the last subsequence $E_{h^{\prime}}=E_{j_{N}}$ of the initial sequence and finite perimeter sets $\left\{F_{j} \subset B_{j}\right\}_{j=1}^{N}$ with

$$
B_{j} \cap E_{h^{\prime}} \rightarrow F_{j}
$$

when $h^{\prime} \rightarrow \infty$ for each $1 \leq j \leq N$. Finally, define $E=A_{0} \cap\left(\bigcup_{j=1}^{N} F_{j}\right)$. Then, by construction

$$
F_{i} \cap B_{i} \cap B_{j} \stackrel{\text { Lebesgue }}{\sim} F_{j} \cap B_{i} \cap B_{j}
$$

and the family $\left\{B_{j}\right\}_{j=1}^{N}$ covers $A_{0}$. Since $E$ is defined by unions and intersection of sets of finite perimeter, it has finite perimeter. It is straightforward to verify the formula for the characteristic function of a finite union. So, in order to prove that $A_{0} \cap E_{h^{\prime}} \rightarrow E$, we note that

$$
\begin{gathered}
1_{A_{0} \cap E_{h^{\prime}}}=1_{A_{0} \cap\left(\cup_{j=1}^{N} B_{j} \cap E_{h^{\prime}}\right)}=1_{A_{0}} 1_{\cup_{j=1}^{N} B_{j} \cap E_{h^{\prime}}}= \\
=1_{A_{0}}\left(1-\sum_{K \subset\{1, \ldots, N\}}(-1)^{|K|} 1_{\cap_{j \in K} B_{j} \cap E_{h^{\prime}}}\right)= \\
\rightarrow^{1.22} 1_{A_{0}}\left(1-\sum_{K \subset\{1, \ldots, N\}}(-1)^{|K|} 1_{\cap_{j \in K} F_{j}}\right)= \\
=1_{A_{0}} 1_{\cup_{j=1}^{N} F_{j}}=1_{E}
\end{gathered}
$$

then $A_{0} \cap E_{h^{\prime}} \rightarrow E$ what implies that $\mu_{A_{0} \cap E_{h^{\prime}}} \stackrel{*}{\rightarrow} \mu_{E}$.

The next theorem gives information about the limit set of a sequence of almost minimizing sets. Indeed, if the sequence locally converges in $L^{1}$ sense to a finite perimeter set, it is possible to state that the limit set 
will be an almost minimizing set as well.

Theorem 1.10. (Closure theorem for sequence of almost minimizing sets) Let $n \geq 2$ and $\left\{E_{h}\right\}_{h \in \mathbb{N}}$ be a sequence with each $E_{h}$ being $\left(\Lambda_{h}, r_{h}\right)-$ minimizing in $A, \Lambda_{h} r_{h} \leq 1, \liminf _{h \rightarrow \infty} r_{h}>0$. If $A_{0} \Subset A$ is an open set with finite perimeter and $A_{0} \cap E_{h} \rightarrow E$, $E$ with finite perimeter. Then $E$ is a $\left(\Lambda, r_{0}\right)-$ minimizing set in $A_{0}$, where $\Lambda=\limsup _{h \rightarrow \infty} \Lambda_{h}, r_{0}=\liminf _{h \rightarrow \infty} r_{h}$.

Proof. Let $F$ be a competitor of $E$, i.e. $F$ is a Caccioppoli with $E \Delta F \Subset$ $\mathrm{B}(x, r) \cap A_{0}, 0<r<\liminf _{h \rightarrow \infty} r_{h} \doteq r_{0}$, we aim to construct one competitor for each $E_{h}$ from $F$. For this purpose, let us state a claim which will be proved later

Claim 1: If $y \in A_{0}, d_{y}=\min \left\{r_{0}, \operatorname{dist}\left(y, \partial A_{0}\right)\right\}$, for almost all $r \in$ $\left(0, d_{y}\right)$, it holds

$$
\begin{gathered}
\mathcal{H}_{n-1}\left(\partial \mathbf{B}(y, r) \cap \partial^{*} F\right)=\mathcal{H}_{n-1}\left(\partial \mathbf{B}(y, r) \cap \partial^{*} E_{h}\right)=0, \forall h \in \mathbb{N} \\
\liminf _{h \rightarrow \infty} \mathcal{H}_{n-1}\left(\partial \mathbf{B}(y, r) \cap\left(E^{(1)} \Delta E_{h}^{(1)}\right)\right)=0
\end{gathered}
$$

Since $E \Delta F$ is compactly contained in $\mathbf{B}(x, r) \cap A_{0}$, it is possible to choose a finite family $\left\{B_{j}=\mathbf{B}\left(y_{j}, r_{j}\right)\right\}_{j=1}^{N}$ such that $y_{j} \in A_{0}$ and $r_{j} \in\left(0, d_{y_{j}}\right)$ satisfying 1.23 and 1.24 and also satisfying

$$
E \Delta F \Subset G \doteq \bigcup_{j=1}^{N} B_{j} \Subset \mathrm{B}(x, r) \cap A_{0}
$$

Now, we are able to construct the competitor of $E_{h}$ that we have looked for. Define

$$
F_{h}=\left(E_{h} \backslash G\right) \bigcup(G \cap F) \quad \text { for each } h \in \mathbb{N}
$$

that is, $F_{h}$ inside $G$ is equal to $F$ and outside $G$ it is equal to $E_{h}$. Thus, it follows

$$
E_{h} \Delta F_{h} \Subset \mathrm{B}(x, r) \cap A_{0} \Subset \mathrm{B}\left(x, r_{h}\right) \cap A_{0}
$$

where $h$ is being taken sufficiently large to guarantee $r<\inf _{h \geq M} r_{h}$ and ensure the second inclusion. Since the space of finite perimeter sets is closed under intersection, union and complement, we have that $F_{h}$ is a set of finite perimeter and, since $\partial G \subset \bigcup_{j=1}^{N} \partial B_{j}$, from 1.23

$$
\mathcal{H}_{n-1}\left(\partial G \cap \partial^{*} F\right)=\mathcal{H}_{n-1}\left(\partial G \cap \partial^{*} E_{h}\right)=0 \quad \forall h \in \mathbb{N}
$$

Claim 2: $E^{(1)} \cap \partial G=F^{(1)} \cap \partial G$

The claim and 1.24 ensure that

$$
\liminf _{h \rightarrow \infty} \mathcal{H}_{n-1}\left(\partial G \cap\left(F^{(1)} \Delta E_{h}^{(1)}\right)\right)=0
$$


Since $A_{0} \Subset A$, from 1.27 , for each $h$ sufficiently large, $F_{h}$ is a competitor for the $\left(\Lambda_{h}, r_{h}\right)$-minimality of $E_{h}$ in $A$, thus

$$
\mathcal{P}\left(E_{h}, \mathbf{B}(x, r)\right) \leq \mathcal{P}\left(F_{h}, \mathbf{B}(x, r)\right)+\Lambda_{h}\left|E_{h} \Delta F_{h}\right|
$$

The equalities in 1.28 allows to apply the comparison theorems for Caccioppoli sets (Theorem 16.16 in [Mag12]). Hence, it follows the equality

$$
\mathcal{P}\left(F_{h}, \mathbf{B}(x, r)\right)=\mathcal{P}(F, G)+\mathcal{P}\left(E_{h}, \mathbf{B}(x, r) \backslash \bar{G}\right)+\mathcal{H}_{n-1}\left(\partial^{*} G \cap\left(E_{h}^{(1)} \Delta F^{(1)}\right)\right)
$$

Adding this equation to the minimality condition of $E_{h}$ and, noting that, since $F_{h} \cap(\mathbf{B}(x, r) \backslash \bar{G})=E_{h} \cap(\mathbf{B}(x, r) \backslash \bar{G})$, we have $\mathcal{P}\left(E_{h}, \mathbf{B}(x, r) \backslash\right.$ $\bar{G})=\mathcal{P}\left(F_{h}, \mathbf{B}(x, r) \backslash \bar{G}\right)$, then it follows

$$
\begin{gathered}
\mathcal{P}\left(E_{h}, \mathbf{B}(x, r)\right) \leq \\
\leq \mathcal{P}(F, G)+\mathcal{P}\left(F_{h}, \mathbf{B}(x, r) \backslash \bar{G}\right)+\mathcal{H}_{n-1}\left(\partial^{*} G \cap\left(E_{h}^{(1)} \Delta F^{(1)}\right)\right)+\Lambda_{h}\left|E_{h} \Delta F_{h}\right|
\end{gathered}
$$

thus, we find that

$$
\begin{gathered}
\liminf _{h \rightarrow \infty} \mathcal{P}\left(E_{h}, \mathbf{B}(x, r)\right)+\liminf _{h \rightarrow \infty}(-\mathcal{P}(F, G))+\liminf _{h \rightarrow \infty}\left(-\mathcal{P}\left(F_{h}, \mathbf{B}(x, r) \backslash \bar{G}\right)\right) \\
+\liminf _{h \rightarrow \infty}\left(-\Lambda_{h}\left|E_{h} \Delta F_{h}\right|\right) \leq \\
\liminf _{h \rightarrow \infty}\left(\mathcal{P}\left(E_{h}, \mathbf{B}(x, r)\right)-\mathcal{P}(F, G)-\mathcal{P}\left(F_{h}, \mathbf{B}(x, r) \backslash \bar{G}\right)-\Lambda_{h}\left|E_{h} \Delta F_{h}\right|\right) \\
\leq \liminf _{h \rightarrow \infty} \mathcal{H}_{n-1}\left(\partial^{*} G \cap\left(E_{h}^{(1)} \Delta F^{(1)}\right)\right)={ }^{*} 0
\end{gathered}
$$

where in $\left(^{*}\right)$ we have used 1.29 and $\partial^{*} G \subset \partial G$. Since $-\liminf _{h \rightarrow \infty}\left(-a_{h}\right)=$ $\limsup a_{h}$ for any $\left(a_{h}\right)$, we obtain

$$
\begin{aligned}
\liminf _{h \rightarrow \infty} \mathcal{P}\left(E_{h}, \mathbf{B}(x, r)\right) & \leq \mathcal{P}(F, G)+\limsup _{h \rightarrow \infty} \mathcal{P}\left(F_{h}, \mathbf{B}(x, r) \backslash \bar{G}\right) \\
+ & \limsup _{h \rightarrow \infty} \Lambda_{h}\left|E_{h} \Delta F_{h}\right|
\end{aligned}
$$

By the definition of $F_{h}$ (1.26), we have $E_{h} \Delta F_{h}=G \cap E_{h} \Delta F$. Hence, since $E_{h} \cap A_{0} \rightarrow E$ and $G \Subset A_{0}(1.27)$, we conclude that $E_{h} \Delta F_{h} \rightarrow$ $G \cap E \Delta F=E \Delta F$. Then, it follows that

$$
\begin{array}{r}
\liminf _{h \rightarrow \infty} \mathcal{P}\left(E_{h}, \mathbf{B}(x, r)\right) \leq \\
\leq \mathcal{P}(F, G)+\limsup _{h \rightarrow \infty} \mathcal{P}\left(F_{h}, \mathbf{B}(x, r) \backslash \bar{G}\right)+\left(\limsup _{h \rightarrow \infty} \Lambda_{h}\right)|E \Delta F|
\end{array}
$$

We note that $F_{h} \rightarrow F$ follows directly by the definition of $F_{h}$ and 1.25 (i.e. outside $G$ the set $F$ is equal to $E$ ), then $\mu_{F_{h}} \stackrel{*}{\rightarrow} \mu_{F}$. As a consequence of this weak-star convergence (Theorem 1.40 in [LCE92]) and the 
compactness of $\overline{\mathbf{B}(x, r)} \backslash G$, it holds that

$$
\begin{gathered}
\limsup _{h \rightarrow \infty} \mathcal{P}\left(F_{h}, \mathbf{B}(x, r) \backslash \bar{G}\right) \leq \limsup _{h \rightarrow \infty} \mathcal{P}\left(F_{h}, \overline{\mathbf{B}(x, r)} \backslash G\right) \leq \\
\leq \mathcal{P}(F, \overline{\mathbf{B}(x, r)} \backslash G)={ }^{*} \mathcal{P}(F, \mathbf{B}(x, r) \backslash G)
\end{gathered}
$$

where $(*)$ follows from 1.23 and $\mathcal{H}_{n-1}\left(\llcorner)_{\partial^{*} F}(\cdot)=\mathcal{P}(F, \cdot)\right.$. The last inequality together with $\mathcal{P}(E, \mathbf{B}(x, r)) \leq \liminf _{h \rightarrow \infty} \mathcal{P}\left(E_{h}, \mathbf{B}(x, r)\right)$ (lowersemicontinuity of the perimeter, Theorem 5.2.2 in [FL03]) and $1.30 \mathrm{im}$ plies

$$
\mathcal{P}(E, \mathbf{B}(x, r)) \leq \mathcal{P}(F, \mathbf{B}(x, r))+\left(\limsup _{h \rightarrow \infty} \Lambda_{h}\right)|E \Delta F|
$$

and the proof is done. Let me prove the claims.

Proof of Claim 1: Lets start with the proof of 1.24, to state that 1.24 is true almost everywhere in $\left(0, d_{y}\right)$, note that

$$
\begin{gathered}
0=^{*} \lim _{h \rightarrow \infty}\left|\mathbf{B}\left(y, d_{y}\right) \cap\left(E \Delta E_{h}\right)\right|=^{* *} \lim _{h \rightarrow \infty}\left|\mathbf{B}\left(y, d_{y}\right) \cap\left(E^{(1)} \Delta E_{h}^{(1)}\right)\right|= \\
={ }^{* *} \lim _{h \rightarrow \infty} \int_{0}^{d_{y}} \mathcal{H}_{n-1}\left(\partial \mathbf{B}(y, r) \cap\left(E^{(1)} \Delta E_{h}^{(1)}\right)\right) \mathrm{d} r
\end{gathered}
$$

where (*) follows from the convergence of $A_{0} \cap E_{h} \rightarrow E$ and $\mathrm{B}\left(y, d_{y}\right) \subset$ $A_{0},(* *)$ is directly checkable from $\left|E \Delta E_{h}\right|=\left|E \Delta E_{h}^{(1)}\right|=\left|E^{(1)} \Delta E_{h}^{(1)}\right|$ and $(* * *)$ is a consequence of the coarea formula, then, by the Fatou's lemma

$$
\begin{aligned}
& \int_{0}^{d_{y}} \liminf _{h \rightarrow \infty} \mathcal{H}_{n-1}\left(\partial \mathbf{B}(y, r) \cap\left(E^{(1)} \Delta E_{h}^{(1)}\right)\right) \leq \\
\leq & \lim _{h \rightarrow \infty} \int_{0}^{d_{y}} \mathcal{H}_{n-1}\left(\partial \mathbf{B}(y, r) \cap\left(E^{(1)} \Delta E_{h}^{(1)}\right)\right) \mathrm{d} r=0
\end{aligned}
$$

thus, 1.24 is valid almost everywhere in the interval. Let $I$ be the subset of $\left(0, d_{y}\right)$ such that $(2.13)$ is valid and denote by $m$ be the Lebesgue measure on the real line. Since $\mathcal{H}_{n-1}\left\llcorner\partial^{*}(\cdot)\right.$ is a Radon measure, we have $m\left(\left\{r \in\left(0, d_{y}\right): \mathcal{H}_{n-1}\left(\partial \mathbf{B}(y, r) \cap \partial^{*} E_{h}\right)=0\right\}\right)=0$ for each $h \geq-1$, where $E_{-1}=F$. But,

$$
\begin{gathered}
J=\left(I \cap\left(\bigcap_{h \geq-1}\left\{r \in\left(0, d_{y}\right): \mathcal{H}_{n-1}\left(\partial \mathbf{B}(y, r) \cap \partial^{*} E_{h}\right)=0\right\}\right)\right)^{c}= \\
=I^{c} \cup\left(\bigcup_{h \geq-1}\left\{r \in\left(0, d_{y}\right): \mathcal{H}_{n-1}\left(\partial \mathbf{B}(y, r) \cap \partial^{*} E_{h}\right)=0\right\}^{c}\right) \Rightarrow \\
\Rightarrow m(J)=0
\end{gathered}
$$

thus, the intersection has total measure in the interval $\left(0, d_{y}\right)$, therefore 1.23 and 1.24 are true almost everywhere in the interval.

Proof of Claim 2: Take $x \in \partial G \cap E^{(1)}$, then $\exists U \subset \overline{(E \Delta F)}^{c}$ open 
set with $x \in U$, thus $1_{E}\left\lfloor_{U} \equiv 1_{F} \iota_{U} \Rightarrow x \in F^{(1)}\right.$, therefore $\partial G \cap E^{(1)} \subset$ $\partial G \cap F^{(1)}$, analogous argument for the reverse inclusion.

Proposition 1.11. Let $\left\{E_{h}\right\}_{h \in \mathbb{N}}, A_{0}$ and $E$ as chosen in the previous theorem, then

$$
\begin{gathered}
\mu_{A_{0} \cap E_{h}} \stackrel{*}{\rightarrow} \mu_{E} \\
\left|\mu_{E_{h}}\right| \stackrel{*}{\rightarrow}\left|\mu_{E}\right| \quad \text { in } \quad A_{0}
\end{gathered}
$$

Moreover, it is true that

(1) If $x_{h} \in A_{0} \in \partial E_{h}, x_{h} \rightarrow x$ and $x \in A_{0}$, then $x \in A_{0} \cap \partial E$;

(2) If $x \in A_{0} \cap \partial E$, then exists $\left\{x_{h}\right\}_{h \in \mathbb{N}}$ such that $x_{h} \in A_{0} \cap \partial E_{h}$ and $x_{h} \rightarrow x$.

Proof. Since $E_{h} \cap A_{0} \rightarrow E$, the convergence in 1.31 follows from the Representation Theorem (Theorem 5.2.1 in [FL03]) which states that $\int 1_{A^{\prime}} \operatorname{div} \phi \mathrm{d} \mathcal{H}_{n}=-\int \phi \mathrm{d}\left(\nu_{A^{\prime}} \mu_{A^{\prime}}\right)$ for all $\phi \in C_{c}^{0}\left(\mathbb{R}^{n}\right)$ and all $A^{\prime}$ finite perimeter set.

Proof of 1.32: First of all, let us suppose that $\left|\mu_{A_{0} \cap E_{h}}\right|^{*} \rightarrow$ in $A_{0}$, we contend that $\mu=\left|\mu_{E}\right|$ in $A_{0}$. Indeed, it holds $\mu(U) \geq\left|\mu_{E}\right|(U), \forall U$ Borel set of $\mathbb{R}^{n}$ (from Proposition 4.30 in [Mag12]). To show the reverse inequality, we will use the construction made in Theorem 1.10. Take $s_{0}<$ $r_{0}$ such that $x \in A_{0}, \mathbf{B}\left(x, s_{0}\right) \Subset A_{0}$ and fix $M$ such that $s_{0}<\inf _{h \geq M} r_{h}$. Define for each $h \geq M$

$$
F_{h}=(E \cap \mathbf{B}(x, s)) \bigcup\left(E_{h} \backslash \mathbf{B}(x, s)\right)
$$

where $s \in\left(0, s_{0}\right)$ is such that

$$
\begin{gathered}
\mathcal{H}_{n-1}\left(\partial \mathbf{B}(x, s) \cap \partial^{*} E\right)=\mathcal{H}_{n-1}\left(\partial \mathbf{B}(x, s) \cap \partial^{*} E_{h}\right)=0 \quad h \geq M \\
\liminf _{h \rightarrow \infty} \mathcal{H}_{n-1}\left(\partial \mathbf{B}(x, s) \cap\left(E^{(1)} \Delta E_{h}^{(1)}\right)\right)=0
\end{gathered}
$$

The fact that the conditions above hold for a.e. $s \in\left(0, s_{0}\right)$ follows from the same argument in the proof of claim 1 in Theorem 1.10. On the other hand, since $E_{h} \Delta F_{h} \Subset \mathrm{B}\left(x, s_{0}\right) \Subset A_{0} \Subset A$, we have

$$
\mathcal{P}\left(E_{h}, \mathbf{B}(x, s)\right) \leq \mathcal{P}\left(F_{h}, \mathbf{B}(x, s)\right)+\Lambda_{h}\left|E_{h} \Delta F_{h}\right|
$$

Therefore, as in the proof of Theorem 1.10, by 1.34, we can use the operations of Caccioppoli sets (Theorem 16.16 in [Mag12]) for $\mathbf{B}\left(x, s_{1}\right) \Subset$ $\mathbf{B}(x, s)$ where we fixed $s_{1} \in\left(0, s_{0}\right)$ such that 1.35 holds true. Then

$$
\begin{gathered}
\mathcal{P}\left(F_{h}, \mathbf{B}(x, s)\right)=\mathcal{P}\left(E, \mathbf{B}\left(x, s_{1}\right)\right)+\mathcal{P}\left(E_{h}, \mathbf{B}(x, s) \backslash \overline{\mathbf{B}\left(x, s_{1}\right)}\right) \\
+\mathcal{H}_{n-1}\left(\partial \mathbf{B}\left(x, s_{1}\right) \cap\left(E^{(1)} \Delta E_{h}^{(1)}\right)\right)
\end{gathered}
$$

Arguing as in the proof of Theorem 1.10, by 1.35, we obtain that

$$
\liminf _{h \rightarrow \infty} \mathcal{P}\left(F_{h}, \mathbf{B}(x, s)\right)+\liminf _{h \rightarrow \infty}-\mathcal{P}\left(E, \mathbf{B}\left(x, s_{1}\right)\right)
$$




$$
\begin{gathered}
+\liminf _{h \rightarrow \infty}-\mathcal{P}\left(E_{h}, \mathbf{B}(x, s) \backslash \overline{\mathbf{B}\left(x, s_{1}\right)}\right) \leq \\
\leq \liminf _{h \rightarrow \infty} \mathcal{H}_{n-1}\left(\partial \mathbf{B}\left(x, s_{1}\right) \cap\left(E^{(1)} \Delta E_{h}^{(1)}\right)\right) \\
\Rightarrow
\end{gathered}
$$

$\liminf _{h \rightarrow \infty} \mathcal{P}\left(F_{h}, \mathbf{B}(x, s)\right) \leq \mathcal{P}\left(E, \mathbf{B}\left(x, s_{1}\right)\right)+\limsup _{h \rightarrow \infty} \mathcal{P}\left(E_{h}, \mathbf{B}(x, s) \backslash \overline{\mathbf{B}\left(x, s_{1}\right)}\right)$

We continue to follow through the steps made in Theorem 1.10. By $\mathbf{B}(x, s) \backslash \overline{\mathbf{B}\left(x, s_{1}\right)} \subset \overline{\mathbf{B}(x, s)} \backslash \mathbf{B}\left(x, s_{1}\right)$, the compactness of $\overline{\mathbf{B}(x, s)} \backslash$ $\mathbf{B}\left(x, s_{1}\right)$, the characterization of weak-convergence of Radon measures (Theorem 1.40 in [LCE92]) and $\mathcal{P}(E, \partial \mathbf{B}(x, s))=0$ (that is 1.34), we find that

$$
\liminf _{h \rightarrow \infty} \mathcal{P}\left(F_{h}, \mathbf{B}(x, s)\right) \leq \mathcal{P}(E, \mathbf{B}(x, s))
$$

By the minimality condition (1.36) and the last inequality, we have that

$$
\begin{gathered}
\liminf _{h \rightarrow \infty} \mathcal{P}\left(E_{h}, \mathbf{B}(x, s)\right)+\liminf _{h \rightarrow \infty}-\Lambda_{h}\left|E_{h} \Delta F_{h}\right| \leq \\
\leq \liminf _{h \rightarrow \infty} \mathcal{P}\left(F_{h}, \mathbf{B}(x, s)\right) \leq \mathcal{P}(E, \mathbf{B}(x, s)) \\
\Rightarrow \\
\liminf _{h \rightarrow \infty} \mathcal{P}\left(E_{h}, \mathbf{B}(x, s)\right) \leq \mathcal{P}(E, \mathbf{B}(x, s))+\limsup _{h \rightarrow \infty} \Lambda_{h}\left|E_{h} \Delta F_{h}\right|
\end{gathered}
$$

By the definition of $F_{h}$ (1.33), we get $E_{h} \Delta F_{h}=E_{h} \Delta E \cap \mathbf{B}(x, s)$. Then, we conclude that

$$
\limsup _{h \rightarrow \infty} \Lambda_{h}\left|E_{h} \Delta F_{h}\right|=\lim _{h \rightarrow \infty} \Lambda_{h}\left|E_{h} \Delta F_{h}\right|=0
$$

because $E_{h} \cap A_{0} \rightarrow E$ and $\mathbf{B}(x, s) \subset A_{0}$. Thus,

$$
\liminf _{h \rightarrow \infty} \mathcal{P}\left(E_{h}, \mathbf{B}(x, s)\right) \leq \mathcal{P}(E, \mathbf{B}(x, s))
$$

Since $\mathbf{B}(x, s) \Subset A_{0}$, we have that

$$
\mathcal{P}\left(E_{h}, \mathbf{B}(x, s)\right)=\left|\mu_{E_{h} \cap A_{0}}\right|(\mathbf{B}(x, s))
$$

Taking into account $\left|\mu_{A_{0} \cap E_{h}}\right| \stackrel{*}{\rightarrow} \mu$ (again with Theorem 1.40 in [LCE92]), the last equality and 1.37 , we establish that

$$
\mu(\mathbf{B}(x, s)) \leq \mathcal{P}(E, \mathbf{B}(x, s))=\left|\mu_{E}\right|(\mathbf{B}(x, s))
$$

Since we have already proved the reverse inequality, given $s_{0}<r_{0}$ such that $\mathbf{B}\left(x, s_{0}\right) \Subset A_{0}$, for almost all $s \in\left(0, s_{0}\right)$, it follows

$$
\left|\mu_{E}\right|(\mathbf{B}(x, s))=\mu(\mathbf{B}(x, s)) .
$$

Then, for almost all $x \in \operatorname{spt} \mu \cap A_{0}$, the Radon-Nikodým derivative exists and, from the last equality, it is equal to 1 , i.e.

$$
\left(D_{\mu}\left|\mu_{E}\right|\right) \equiv 1
$$


Since $\mu \geq\left|\mu_{E}\right|$ in all Borel sets of $\mathbb{R}^{n}$, it is clear that $\left|\mu_{E}\right| \ll \mu$, thus $\left|\mu_{E}\right|$ has no singular part with respect to $\mu$. Therefore, by the LebesgueBesicovitch Differentiation Theorem

$$
\left|\mu_{E}\right|=\left(D_{\mu}\left|\mu_{E}\right|\right) \mu=\mu \quad \text { in } \quad A_{0}
$$

Since $\mu_{E_{h}} L_{A_{0}}=\mu_{E_{h} \cap A_{0}} L_{A_{0}}$ directly implies $\left|\mu_{E_{h}}\right|\left\lfloor_{A_{0}}=\left|\mu_{E_{h} \cap A_{0}}\right| L_{A_{0}}\right.$, we find that $\left|\mu_{A_{0} \cap E_{h}}\right| \stackrel{*}{\rightarrow} \mu=\left|\mu_{E}\right|$ in $A_{0}$. It remains to prove the existence of a Radon measure $\mu$ in $A_{0}$ such that $\left|\mu_{A_{0} \cap E_{h}}\right|^{*} \rightarrow \mu$ in $A_{0}$. That follows directly from the compactness criterion for Radon measures (de la Vallée Poussin's theorem - Theorem 1.41 in [LCE92]). In order to apply the aforementioned theorem, we must verify that $\sup _{h \geq M}\left|\mu_{E_{h} \cap A_{0}}\right|(K)<$ $\infty, \forall K$ compact subset of $A_{0}$. Indeed, given $K \subset A_{0}$ compact, we obtain that

$$
\begin{gathered}
\mathcal{P}\left(E_{h} \cap A_{0}, K\right) \leq \mathcal{P}\left(E_{h} \cap A_{0}, \mathbb{R}^{n}\right) \leq^{*} \mathcal{P}\left(E_{h}, A_{0}\right)+\mathcal{P}\left(A_{0}, \mathbb{R}^{n}\right) \leq \\
\leq^{* *} \sum_{j=1}^{N} \mathcal{P}\left(E_{h}, B_{j}\right)+\mathcal{P}\left(A_{0}, \mathbb{R}^{n}\right) \leq \\
\leq^{* * *} 3 n \omega_{n-1} \sum_{j=1}^{N} r_{j}^{n-1}+\mathcal{P}\left(A_{0}, \mathbb{R}^{n}\right) \leq 3 N n \omega_{n-1} r_{0}^{n-1}+\mathcal{P}\left(A_{0}, \mathbb{R}^{n}\right) \Rightarrow \\
\Rightarrow \sup _{h \geq M} \mathcal{P}\left(E_{h} \cap A_{0}, K\right)<\infty
\end{gathered}
$$

where in $(*)$ it was used the operations with Caccioppoli sets, in $(* *)$ it was taken an open cover of $A_{0}$ by balls $B_{j}=\mathbf{B}\left(x_{j}, r_{j}\right)$, all of them compactly contained in $A$ with $x_{j} \in A_{0} \subset A, r_{j}<s_{0}<r_{0}$ and $s_{0}$ as previously chosen. And in $(* * *)$ we have used the upper inequality in 1.12 and the Remark 1.6.

Proof of (1): For each $h \in \mathbb{N}$, we take $x_{h} \in \partial E_{h} \cap A_{0}$ with $x_{h} \rightarrow x \in$ $A_{0}$. Let $s \in\left(0, r_{0}\right), \mathbf{B}(x, s) \Subset A_{0}$. We set $M \in \mathbb{N}$ in view of $s<\inf _{h \geq M} r_{h}$ and $\mathbf{B}\left(x_{h}, \frac{s}{2}\right) \subset \mathbf{B}(x, s)$. Therefore,

$$
\begin{gathered}
\mathcal{P}(E, \overline{\mathbf{B}(x, s)}) \geq{ }^{*} \limsup _{h \rightarrow \infty} \mathcal{P}\left(E_{h}, \overline{\mathbf{B}(x, s)}\right) \geq \limsup _{h \rightarrow \infty} \mathcal{P}\left(E_{h}, \mathbf{B}(x, s)\right) \geq \\
\geq \limsup _{h \rightarrow \infty} \mathcal{P}\left(E_{h}, \mathbf{B}\left(x_{h}, \frac{s}{2}\right)\right) \geq^{* *} c(n)\left(\frac{s}{2}\right)^{n-1}>0
\end{gathered}
$$

where, since $\overline{\mathbf{B}(x, s)}$ is compact, $(*)$ follows from 1.32 and the characterization of weak-convergence of Radon measures (Theorem 1.40 in [LCE92]). Since $x_{h} \in \partial E_{h}$ and each $E_{h}$ is a $\left(\Lambda, r_{0}\right)-$ minimizing, (**) follows from the lower density estimate in 1.12. So, by the last inequality and the arbitrariness of $s$, we have that $\mathcal{P}(E, \overline{\mathbf{B}(x, s)})>0, \forall s \in\left(0, r_{0}\right)$, thus, $x \in \operatorname{spt} \mu_{E}=\partial E$.

Proof of (2): We would like to highlight this argument because it is independent of the minimality condition of the sets $E_{h}$, i.e. we will only use the weak convergence of the Radon measures. We fix $x \in \partial E \cap A_{0}$ 
and suppose that exists $\epsilon>0, h \geq 0$ such that $\left(\operatorname{spt} \mu_{E_{h^{\prime}}} \cap A_{0}\right) \cap \mathbf{B}(x, \epsilon)=$ $\emptyset, \forall h^{\prime} \geq h$, i.e. $x$ is not in the support of the measure $\mu_{E_{h^{\prime}}}, \forall h^{\prime} \geq$ $h$. From 1.31 and the characterization of weak-convergence of Radon measures (Theorem 1.40 in [LCE92]), it follows that $\mu_{E}^{j}(\mathbf{B}(x, \epsilon)) \leq$ $\liminf _{h^{\prime} \rightarrow \infty} \mu_{E_{h^{\prime}} \cap A_{0}}^{j}(\mathbf{B}(x, \epsilon))=0, \forall j \in\{1, \ldots, n\}$ where we have used the following notation $\mu=\left(\mu^{1}, \ldots, \mu^{n}\right)$. Thus, $x \notin \operatorname{spt} \mu_{E}=\partial E$ what is a contradiction with our choice of $x$. Then, exists a subsequence with the aimed properties. 


\section{Excess theory}

\subsection{Excess and its basic properties}

In this section, we will define and prove results on the Excess theory. This theory is a fundamental point and one of the most significant tools in the regularity theory. The Geometric Measure Theory has evolved along three main branches, they are Currents, Varifolds and Caccioppoli sets. In all of them, the excess turns out to be the main tool used in the regularity theory of minimal or almost minimal sets.

Definition 2.1. Let $E \subset \mathbb{R}^{n}$ be a Caccioppoli, $x \in \mathbb{R}^{n}, \nu \in \mathbb{S}^{n-1}$ and $r>0$. It is called cylindrical excess of $E$ in $x$ with direction $\nu$ in scale $r$ the following number

$$
\boldsymbol{e}(E, x, r, \nu)=\frac{1}{r^{n-1}} \int_{\boldsymbol{C}(x, r, \nu) \cap \partial^{*} E} \frac{\left|\nu-\nu_{E}(y)\right|^{2}}{2} \mathrm{~d} \mathcal{H}_{n-1}(y)
$$

Remark 2.2. The cylindrical excess of $E$ in $x$ with direction $\nu$ in scale $r>0$ can be seen, intuitively, as a measure of how much the reduced boundary of $E$ is far from being a hyperplane passing through $x$ with normal vector $\nu$ inside a cylinder of radius $r$. If we consider the problem of finding the direction in which $E$ is nearest to being a hyperplane, the spherical excess shows the answer

Definition 2.3. Let $E \subset \mathbb{R}^{n}$ be a Caccioppoli, $x \in \mathbb{R}^{n}, \nu \in \mathbb{S}^{n-1}$ and $r>0$. It is called spherical excess of $E$ in $x$ of scale $r$ the following number

$$
\boldsymbol{e}(E, x, r)=\min _{\nu \in \mathbb{S}^{n-1}} \frac{1}{r^{n-1}} \int_{\boldsymbol{B}(x, r) \cap \partial^{*} E} \frac{\left|\nu-\nu_{E}(y)\right|^{2}}{2} \mathrm{~d} \mathcal{H}_{n-1}(y)
$$

Henceforth, we will prove and comment on some basic properties of the excess which will be used recurrently in this work. Unless otherwise explicitly stated, we will refer by excess the cylindrical excess and will make it evident and clear when talking about the spherical excess. The next result is the precise statement of the idea in Remark 2.2. 
Proposition 2.4. Let $E \subset \mathbb{R}^{n}$ be a Caccioppoli with spt $\mu_{E}=\partial E$, $x \in \partial E, r>0$ and $\nu \in \mathbb{S}^{n-1}$, then

$\boldsymbol{e}(E, x, r, \nu)=0 \Leftrightarrow E \cap \boldsymbol{C}(x, r, \nu) \stackrel{\text { Lebesgue }}{\sim}\{y \in \boldsymbol{C}(x, r, \nu):(y-x) \cdot \nu \leq 0\}$

Proof. $(\Leftarrow)$ Since both sets are equivalent, we have $\mu_{E}=\mu_{\{y \in \mathbf{C}(x, r, \nu):(y-x) \cdot \nu \leq 0\}}$ (by Proposition 0.1) what implies

$$
\partial^{*} E \cap \mathbf{C}(x, r, \nu) \stackrel{\mathcal{H}_{n-1}}{\sim}\{y \in \mathbf{C}(x, r, \nu):(y-x) \cdot \nu=0\}
$$

what ensures that $\nu_{E}=\nu_{\{y \in \mathbf{C}(x, r, \nu):(y-x) \cdot \nu=0\}}=\nu$ holds $\mathcal{H}_{n-1}$-almost everywhere in $\partial^{*} E \cap\{y \in \mathbf{C}(x, r, \nu):(y-x) \cdot \nu=0\} \cap \mathbf{C}(x, r, \nu)$, then it follows from the definition of excess that

$$
\begin{gathered}
\mathbf{e}(E, x, r, \nu)=\frac{1}{r^{n-1}} \int_{\mathbf{C}(x, r, \nu) \cap \partial^{*} E} \frac{\left|\nu-\nu_{E}(y)\right|^{2}}{2} \mathrm{~d} \mathcal{H}_{n-1}(y)= \\
={ }^{2.1} \frac{1}{r^{n-1}} \int_{\{y \in \mathbf{C}(x, r, \nu):(y-x) \cdot \nu=0\}} \frac{|\nu-\nu|^{2}}{2} \mathrm{~d} \mathcal{H}_{n-1}(y)=0
\end{gathered}
$$

$(\Rightarrow)$ Since $\mathbf{e}(E, x, r, \nu)=0$ it follows that $\nu_{E}=\nu \mu_{E}$-almost everywhere in $\mathbf{C}(x, r, \nu)$. So, by the same argument used in the proof of Proposition 15.15 in [Mag12], there is $\alpha \in \mathbb{R}$ such that

$$
E \cap \mathbf{C}(x, r, \nu) \stackrel{\text { Lebesgue }}{\sim}\{y \in \mathbf{C}(x, r, \nu):(y-x) \cdot \nu<\alpha\} .
$$

If $\alpha<0$ (respectively, $\alpha>0$ ), $\exists r_{0}>0$ such that $\left|E \cap \mathbf{B}\left(x, r_{0}\right)\right|=0$ (respectively, $\left.\left|E \cap \mathbf{B}\left(x, r_{0}\right)\right|=\omega_{n} r_{0}^{n}\right)$ what is a contradiction with the fact that

$$
x \in \operatorname{spt} \mu_{E}=\partial E=\left\{y \in \mathbb{R}^{n}: 0<|E \cap \mathbf{B}(x, r)|<\omega_{n} r^{n} \text { for all } r>0\right\}
$$

(Proposition 3.1 in [Giu84]). Then $\alpha=0$.

Proposition 2.5. (Excess and Changes of Scale) Let $E \subset \mathbb{R}^{n} a$ Caccioppoli, $x \in \mathbb{R}^{n}, r>s>0$ and $\nu \in \mathbb{S}^{n-1}$, then

$$
e(E, x, s, \nu) \leq\left(\frac{r}{s}\right)^{n-1} e(E, x, r, \nu)
$$

Proof. Since $\mathbf{C}(x, s, \nu) \subset \mathbf{C}(x, r, \nu)$, we have

$$
\begin{aligned}
& \frac{1}{s^{n-1}} \int_{\mathbf{C}(x, s, \nu) \cap \partial^{*} E} \frac{\left|\nu-\nu_{E}(y)\right|^{2}}{2} \mathrm{~d} \mathcal{H}_{n-1}(y) \leq \\
& \leq \frac{1}{s^{n-1}} \int_{\mathbf{C}(x, r, \nu) \cap \partial^{*} E} \frac{\left|\nu-\nu_{E}(y)\right|^{2}}{2} \mathrm{~d} \mathcal{H}_{n-1}(y)
\end{aligned}
$$

It suffices to multiply and divide by $r^{n-1}$ on the right side of the inequality.

Proposition 2.6. (Excess and Blow-up) Let $E \subset \mathbb{R}^{n}$ be a Cacciop- 
poli, $x \in \partial E, r>0$ and $\nu \in \mathbb{S}^{n-1}$, then, for all $s>0$

$\boldsymbol{e}(E, x, r, \nu)=e\left(E_{x_{0}, s}, \frac{x-x_{0}}{s}, \frac{r}{s}, \nu\right) \quad e(E, x, r)=e\left(E_{x_{0}, s}, \frac{x-x_{0}}{s}, \frac{r}{s}\right)$

Proof. Note that $\left|\nu-\nu_{E}\right|^{2}=\left(\nu-\nu_{E}\right) \cdot\left(\nu-\nu_{E}\right)=2-2 \nu \cdot \nu_{E}$. Let $\phi(z)=s z+x_{0}, \forall z \in \mathbb{R}^{n}$, one consequence of the definition of pushforward is

$$
\mu_{E}=s^{n-1} \phi \sharp \mu_{E_{x_{0}, s}} \quad\left|\mu_{E}\right|=s^{n-1} \phi \sharp\left|\mu_{E_{x_{0}, s}}\right|
$$

Putting it all together

$$
\begin{gathered}
\mathbf{e}(E, x, r, \nu)=\frac{1}{r^{n-1}} \int_{\mathbf{C}(x, r, \nu) \cap \partial^{*} E}\left(1-\nu \cdot \nu_{E}\right) \mathrm{d} \mathcal{H}_{n-1}= \\
={ }^{*} \frac{1}{r^{n-1}}\left(\left|\mu_{E}\right|(\mathbf{C}(x, r, \nu))-\nu \cdot \mu_{E}(\mathbf{C}(x, r, \nu))\right)= \\
={ }^{* *} \frac{1}{r^{n-1}}\left(s^{n-1} \phi \sharp\left|\mu_{E_{x_{0}, s}}\right|(\mathbf{C}(x, r, \nu))-s^{n-1} \nu \cdot \phi \sharp \mu_{E_{x_{0}, s}}(\mathbf{C}(x, r, \nu))\right)= \\
=\left(\frac{s}{r}\right)^{n-1}\left(\left|\mu_{E_{x_{0}, s}}\right|\left(\phi^{-1}(\mathbf{C}(x, r, \nu))\right)-\nu \cdot \mu_{E_{x_{0}, s}}\left(\phi^{-1}(\mathbf{C}(x, r, \nu))\right)\right)= \\
={ }^{* * *}\left(\frac{1}{\frac{r}{s}}\right)^{n-1}\left(\left|\mu_{E_{x_{0}, s}}\right|\left(\mathbf{C}\left(\frac{x-x_{0}}{s}, \frac{r}{s}, \nu\right)\right)-\nu \cdot \mu_{E_{x_{0}, s}}\left(\mathbf{C}\left(\frac{x-x_{0}}{s}, \frac{r}{s}, \nu\right)\right)\right)= \\
={ }^{* * * *} \mathbf{e}\left(E_{x_{0}, s}, \frac{x-x_{0}}{s}, \frac{r}{s}, \nu\right)
\end{gathered}
$$

where $(*)$ is a consequence of the De Giorgi's Structure Theorem, $(* *)$ follows from 2.2, (***) it is easy to see that $\mathbf{C}\left(x-x_{0}, \frac{r}{s}, \nu\right)=\phi^{-1}(\mathbf{C}(x, r, \nu))$ and $(* * * *)$ it suffices to reproduce the same argument used in the firsts two equalities. To prove the second equality, note that $\left|\nu-\nu_{E}\right|^{2}$ reaches the minimum in $\nu=\nu_{E}$, thus

$$
\mathbf{e}(E, x, r)=\min _{\nu \in \mathbb{S}^{n-1}} \frac{1}{r^{n-1}}\left(\left|\mu_{E}\right|(\mathbf{C}(x, r, \nu))-\nu \cdot \mu_{E}(\mathbf{C}(x, r, \nu))\right)
$$

It holds

$$
\mathbf{e}(E, x, r)=\frac{\left|\mu_{E}\right|(\mathbf{B}(x, r))}{r^{n-1}}\left(1-\frac{\left|\mu_{E}(\mathbf{B}(x, r))\right|}{\left|\mu_{E}\right|(\mathbf{B}(x, r))}\right)
$$

Note that

$$
\phi^{-1}(\mathbf{B}(x, r))=\mathbf{B}\left(\frac{x-x_{0}}{s}, \frac{r}{s}\right)
$$

With the same argument, we can conclude the proof of the second equality on the Proposition.

Proposition 2.7. Let $E \subset \mathbb{R}^{n}$ be a Caccioppoli, $x \in \partial E, r>0, \nu \in \mathbb{S}^{n-1}$ 
and $T: \mathbb{R}^{n} \rightarrow \mathbb{R}^{n}$ an isometry. Then

$$
\boldsymbol{e}(T(E), x, r, \nu)=\boldsymbol{e}\left(E, T^{-1}(x), r, T^{-1}(\nu)\right)
$$

Proof. Since

$\mathbf{e}(E, x, r, \nu)=\frac{1}{r^{n-1}}\left(\left|\mu_{E}\right|(\mathbf{C}(x, r, \nu))-\nu \cdot \nu_{E} \mathcal{H}_{n-1}\left(\partial^{*} E \cap \mathbf{C}(x, r, \nu)\right)\right)$

$\mu_{T(E)}=T \sharp \mu_{E}$ and $\left|\mu_{T(E)}\right|=T \sharp\left|\mu_{E}\right|$ (Proposition 2.51 in [VOL]), we can verify that

$$
\begin{gathered}
\mathbf{e}(T(E), x, r, \nu)= \\
\frac{1}{r^{n-1}}\left(\left|\mu_{T(E)}\right|(\mathbf{C}(x, r, \nu))-\nu \cdot \nu_{T(E)} \mathcal{H}_{n-1}\left(\partial^{*} T(E) \cap \mathbf{C}(x, r, \nu)\right)\right)= \\
=^{*} \frac{1}{r^{n-1}}\left(T \sharp\left|\mu_{E}\right|(\mathbf{C}(x, r, \nu))-\nu \cdot T\left(\nu_{E} \circ T^{-1}\right) \mathcal{H}_{n-1}\left(\partial^{*} E \cap T^{-1}(\mathbf{C}(x, r, \nu))\right)\right)= \\
\frac{1}{r^{n-1}}\left(\left|\mu_{E}\right|\left(T^{-1}(\mathbf{C}(x, r, \nu))\right)-T^{-1}(\nu) \cdot \nu_{E} \circ T^{-1} \mathcal{H}_{n-1}\left(\partial^{*} E \cap T^{-1}(\mathbf{C}(x, r, \nu))\right)\right)= \\
=\mathbf{e}\left(E, T^{-1}(x), r, T^{-1}(\nu)\right)
\end{gathered}
$$

where in $\left(^{*}\right)$ we have used that $\mathcal{H}_{n-1}(T(A))=\mathcal{H}_{n-1}(A)$ for any $A \subset \mathbb{R}^{n}$ which follows from the properties of the Hausdorff measure (Theorem 2.2 in [LCE92]) and that $T$ is an isometry.

We pointed out that the reduced boundary can be seen as the regular part of the boundary. The next result provides a new interpretation of this fact. Indeed, if we choose a point $x$ of the reduced boundary, the spherical excess at $x$ tends to zero as the scale goes to zero. Then, we can find both a direction and a scale provided the excess at $x$ is as small as we want.

Proposition 2.8. Let $E \subset \mathbb{R}^{n}$ be a Caccioppoli and $x \in \partial^{*} E$. Then $\lim _{r \rightarrow 0^{+}} e(E, x, r)=0$. Moreover, $\forall \epsilon>0, \exists \nu \in \mathbb{S}^{n-1}, \exists r>0$ such that $e(E, x, r, \nu) \leq \epsilon$.

Proof. By the Remark of Definition 5.4 and (iii) of Theorem 5.14 both in [LCE92], we have that

$$
\begin{gathered}
\lim _{r \rightarrow 0^{+}}\left|\frac{\mu_{E}(\mathbf{B}(x, r))}{\left|\mu_{E}\right|(\mathbf{B}(x, r))}\right|=\left|\nu_{E}(x)\right|=1 \\
\lim _{r \rightarrow 0^{+}} \frac{\left|\mu_{E}\right|(\mathbf{B}(x, r))}{\omega_{n-1} r^{n-1}}=\lim _{r \rightarrow 0^{+}} \frac{\mathcal{P}(E, \mathbf{B}(x, r))}{\omega_{n-1} r^{n-1}}=1
\end{gathered}
$$

Joining these two limits in 2.3, it follows that $\lim _{r \rightarrow 0^{+}} \mathbf{e}(E, x, r)=0$. Moreover, given $\epsilon>0$, take $r$ such that $\mathbf{e}(E, x, r) \leq \epsilon$. Since

$$
\mathbf{C}\left(x, \frac{s}{\sqrt{2}}, \nu\right) \subset \mathbf{B}(x, s)
$$


is valid for all $s>0$, in particular, for $r$, we obtain that

$$
\mathbf{e}\left(E, x, \frac{r}{\sqrt{2}}, \nu\right) \leq\left(\frac{1}{\frac{r}{\sqrt{2}}}\right)^{n-1} \int_{\mathbf{B}(x, r) \cap \partial^{*} E} \frac{\left|\nu-\nu_{E}\right|^{2}}{2} \mathrm{~d} \mathcal{H}_{n-1} \leq \sqrt{2}^{n-1} \epsilon
$$

Up to now, we did not state anything about how the excess behaves with changes of the direction. For this purpose, we will be able to state some result only in the case of the almost minimizing sets as follows.

Proposition 2.9. (Change on the direction) If $n \geq 2$, exists a constant $C(n)$ such that for all $E\left(\Lambda, r_{0}\right)$-minimizing set in $A$ with $\Lambda r_{0} \leq 1$ and for all $x \in \partial E \cap A, \sqrt{2} r<r_{0}, \boldsymbol{B}(x, 2 r) \Subset A, \nu, \nu_{0} \in \mathbb{S}^{n-1}$, it holds

$$
e(E, x, r, \nu) \leq C_{d}(n)\left(e\left(E, x, \sqrt{2} r, \nu_{0}\right)+\left|\nu-\nu_{0}\right|^{2}\right)
$$

Proof. Since $f(x)=|x|^{2}$ is a convex function, taking $x=\nu_{0}-\nu_{E}, y=$ $\nu-\nu_{0}, t=\frac{1}{2}$ in the inequality $f(t x+(1-t) y) \leq t f(x)+(1-t) f(y)$ and $\mathbf{C}(x, r, \nu) \subset \mathbf{C}\left(x, \sqrt{2} r, \nu_{0}\right)$, then

$$
\begin{gathered}
\mathbf{e}(E, x, r, \nu) \leq \\
\frac{2}{r^{n-1}} \int_{\mathbf{C}\left(x, \sqrt{2} r, \nu_{0}\right) \cap \partial^{*} E}\left(\frac{\left|\nu_{0}-\nu_{E}\right|^{2}}{2}+\frac{\left|\nu-\nu_{0}\right|^{2}}{2}\right) \mathrm{d} \mathcal{H}_{n-1}= \\
={ }^{*} 2 \sqrt{2}^{n-1}\left(\mathbf{e}\left(E, x, \sqrt{2} r, \nu_{0}\right)+\frac{\mathcal{P}\left(E, \mathbf{C}\left(x, \sqrt{2} r, \nu_{0}\right)\right)}{2(\sqrt{2} r)^{n-1}}\left|\nu-\nu_{0}\right|^{2}\right) \leq \\
\leq^{* *} 2 \sqrt{2}^{n-1}\left(\mathbf{e}\left(E, x, \sqrt{2} r, \nu_{0}\right)+\frac{3 n \omega_{n-1}}{2}\left|\nu-\nu_{0}\right|^{2}\right)
\end{gathered}
$$

where in $(*)$ it was used that the function in the integral is constant together with the De Giorgi's Structure Theorem and $(* *)$ follows from Corollary 1.8. It suffices to take $C_{d}(n)=2 \sqrt{2}^{n-1} 3 n \omega_{n-1}$.

The next result, shortly, state that when restricted to the almost minimizing sets, it is possible to affirm that the excess can not be small in two opposite directions.

Proposition 2.10. Let $E$ be a $\left(\Lambda, r_{0}\right)$ - minimizing in $A, x \in \partial E, \nu \in$ $\mathbb{S}^{n-1}, r<r_{0}, C(x, r, \nu) \subset A$, then

$$
\boldsymbol{e}(E, x, r, \nu)+\boldsymbol{e}(E, x, r,-\nu) \geq 2 c(n)
$$

where $c(n)$ is the constant from the density estimates.

Proof. Note that $\left(\nu_{E}-\nu\right) \cdot\left(\nu_{E}+\nu\right)=0$, by Pitagoras' Theorem $\mid \nu_{E}-$ $\left.\nu\right|^{2}+\left|\nu_{E}+\nu\right|^{2}=4|\nu|^{2}=4$, then

$$
\mathbf{e}(E, x, r, \nu)+\mathbf{e}(E, x, r,-\nu)=\frac{1}{r^{n-1}} \int_{\partial^{*} E \cap \mathbf{C}(x, r, \nu)} \frac{4}{2} \mathrm{~d} \mathcal{H}_{n-1}=
$$




$$
\begin{gathered}
=\frac{2 \mathcal{P}(E, \mathbf{C}(x, r, \nu))}{r^{n-1}} \geq \\
\geq \frac{2 \mathcal{P}(E, \mathbf{B}(x, r))}{r^{n-1}} \geq^{*} 2 c(n)
\end{gathered}
$$

where $(*)$ it was used the lower inequality in $(2.2)$.

One result that will be used frequently and, usually, together with the compactness and closure theorems, is the lower semi-continuity of the excess. That is, what we can extract of the excess of a sequence of almost minimizing sets under some assumptions. For this purpose, let me state a preliminary result which deal with the continuity of the excess under some restrictive conditions.

Proposition 2.11. Let $A, A_{0}$ open sets of $\mathbb{R}^{n}$ with $A_{0} \Subset A, \mathcal{P}\left(A_{0}, \mathbb{R}^{n}\right)<$ $\infty$. Suppose that $\left\{E_{h}\right\}_{h \in \mathbb{N}}$ is a sequence of $\left(\Lambda, r_{0}\right)$-minimizing sets in $A$ with $\Lambda r_{0} \leq 1$ and such that $A_{0} \cap E_{h} \rightarrow E$. Then, for all $C(x, r, \nu) \Subset A_{0}$ satisfying

$$
\mathcal{H}_{n-1}\left(\partial^{*} E \cap \partial \boldsymbol{C}(x, r, \nu)\right)=0
$$

it holds

$$
\boldsymbol{e}(E, x, r, \nu)=\lim _{h \rightarrow \infty} \boldsymbol{e}\left(E_{h}, x, r, \nu\right)
$$

Proof. We have stated in Proposition 1.11 that

$$
\begin{gathered}
\mu_{A_{0} \cap E_{h}} \stackrel{*}{\rightarrow} \mu_{E} \\
\left|\mu_{A_{0} \cap E_{h}}\right| \stackrel{*}{\rightarrow}\left|\mu_{E}\right| \quad \text { in } \quad A_{0}
\end{gathered}
$$

By Theorem 1.40 in [LCE92], $\mathcal{H}_{n-1}\left(\partial^{*} E \cap \partial \mathbf{C}(x, r, \nu)\right)=0$ implies

$$
\left|\mu_{E}\right|(\mathbf{C}(x, r, \nu))=\lim _{h \rightarrow \infty}\left|\mu_{E_{h}}\right|(\mathbf{C}(x, r, \nu))
$$

and

$$
\mu_{E}(\mathbf{C}(x, r, \nu))=\lim _{h \rightarrow \infty} \mu_{E_{h}}(\mathbf{C}(x, r, \nu))
$$

Since $\mathbf{C}(x, r, \nu) \Subset A_{0}$ it holds

$$
\mu_{A_{0} \cap E_{h}}(\mathbf{C}(x, r, \nu))=\mu_{E_{h}}(\mathbf{C}(x, r, \nu))
$$

Putting it all together and combining with the fact that, $\forall F$ Caccioppoli

$$
\mathbf{e}(F, x, r, \nu)=\frac{1}{r^{n-1}}\left(\left|\mu_{F}\right|(\mathbf{C}(x, r, \nu))-\nu \cdot \mu_{F}(\mathbf{C}(x, r, \nu))\right)
$$

the result follows.

Theorem 2.12. (Lower semi-continuity of the excess) Let $A, A_{0}$ opens of $\mathbb{R}^{n}$ with $A_{0} \Subset A, \mathcal{P}\left(A_{0}, \mathbb{R}^{n}\right)<\infty$. Suppose that $\left\{E_{h}\right\}_{h \in \mathbb{N}}$ is a sequence of $\left(\Lambda, r_{0}\right)$ - minimizing sets in $A$ with $\Lambda r_{0} \leq 1$ and such that $A_{0} \cap E_{h} \rightarrow E$. Then, for all $\boldsymbol{C}(x, r, \nu) \Subset A_{0}$ it holds

$$
\boldsymbol{e}(E, x, r, \nu) \leq \liminf _{h \rightarrow \infty} \boldsymbol{e}\left(E_{h}, x, r, \nu\right)
$$


Proof. Recalling the following equalities

$$
\begin{gathered}
\mathbf{e}(E, x, r, \nu)=\frac{1}{r^{n-1}} \int_{\mathbf{C}(x, r, \nu) \cap \partial^{*} E}\left(1-\nu \cdot \nu_{E}\right) \mathrm{d} \mathcal{H}_{n-1}= \\
=\frac{1}{r^{n-1}}\left(\left|\mu_{E}\right|(\mathbf{C}(x, r, \nu))-\nu \cdot \mu_{E}(\mathbf{C}(x, r, \nu))\right)
\end{gathered}
$$

By the continuity from below of the measure $\mathcal{H}_{n-1\left\llcorner\partial^{*} E\right.}$ (Theorem 1.8 in [Fol99]) and the continuity of $r \mapsto r^{n-1}$, it is easy to see that $r \mapsto$ $\mathbf{e}(E, x, r, \nu)$ is left-continuous, that is

$$
\mathbf{e}(E, x, r, \nu)=\lim _{s \rightarrow r^{-}} \mathbf{e}(E, x, s, r)
$$

Since $\mathcal{H}_{n-1\left\llcorner\partial^{*} E\right.}(\mathbf{C}(x, r, \nu))<\infty$, exists a sequence $\left\{r_{k}\right\}_{k \in \mathbb{N}}$ such that $r_{k} \rightarrow r^{-}$and $\mathcal{H}_{n-1}\left(\partial^{*} E \cap \partial \mathbf{C}\left(x, r_{k}, \nu\right)\right)=0, \forall k \in \mathbb{N}$. The changing of scale of the excess (Proposition 2.5) yields

$$
\mathbf{e}\left(E_{h}, x, r_{k}, \nu\right) \leq\left(\frac{r}{r_{k}}\right)^{n-1} \mathbf{e}\left(E_{h}, x, r, \nu\right)
$$

From the previous proposition and the choice of the sequence $\left\{r_{k}\right\}_{k \in \mathbb{N}}$, it holds for each $k$

$$
\mathbf{e}\left(E, x, r_{k}, \nu\right)=\mathbf{e}\left(E_{h}, x, r_{k}, \nu\right)
$$

The thesis is achieved by taking the $\liminf _{k \rightarrow \infty}$ in the inequality (3.4), the left-continuity of $r \mapsto \mathbf{e}(E, x, r, \nu)$ and applying the last equality.

\subsection{Bounded excess consequences}

In this section, our goal is to describe locally (in general, up to $H^{n}$ equivalence) the almost minimizing sets under the assumption, which is crucial for the development of the regularity theory, of the boundedness of the excess. Under this assumption, how much information is it possible to infer concerning the local regularity of both topological and reduced boundaries? The first result of this section describes the almost minimizing sets with bounded excess inside a cylinder. From now on, we will always suppose that $n \geq 2$ and $e_{n}=(0,0, \ldots, 0,1) \in \mathbb{R}^{n}$.

Theorem 2.13. (Small-excess position) Given $t_{0} \in(0,1)$, exists a positive constant $\omega\left(n, t_{0}\right)$ such that, for all $E$ being a $\left(\Lambda, r_{0}\right)$-minimizing 
in $C\left(0,2, e_{n}\right)$ with $\Lambda r_{0} \leq 1,0 \in \partial E$ and

$$
e\left(E, 0,2, e_{n}\right) \leq \omega\left(n, t_{0}\right)
$$

it holds:

$$
\begin{gathered}
|\boldsymbol{q} x|<t_{0} \quad \forall x \in \boldsymbol{C}\left(0,1, e_{n}\right) \cap \partial E \\
\left\{x \in \boldsymbol{C}\left(0,1, e_{n}\right) \cap E: \boldsymbol{q} x>t_{0}\right\}=\emptyset \\
\left\{x \in \boldsymbol{C}\left(0,1, e_{n}\right) \backslash E: \boldsymbol{q} x<-t_{0}\right\}=\emptyset
\end{gathered}
$$

Proof. This proof will be done by contradiction. That is, given $t_{0} \in$ $(0,1)$, for each constant $\omega\left(n, t_{0}\right)>0$, exists $E$ in the conditions above (in the hypothesis of the theorem) such that $E$ does not satisfy one of the equations 2.6, 2.7 or 2.8. Therefore, we will take a sequence of positive constants tending to zero, $\omega_{h}\left(n, t_{0}\right)$, and $E_{h}$ the set in the conditions above referent to this constant. It holds:

$$
\Lambda r_{0} \leq 1 \quad \lim _{h \rightarrow \infty} \mathbf{e}\left(E_{h}, 0,2, e_{n}\right)=0 \quad 0 \in \partial E_{h}, \forall h \in \mathbb{N}
$$

at least one of the equations $2.6,2.7$ or 2.8 , does not hold to an infinite number of $E_{h}$, otherwise the sequence will not be infinite. Since $\mathbf{C}\left(0, \frac{5}{3}, e_{n}\right) \Subset \mathbf{C}\left(0,2, e_{n}\right)$ and $\mathcal{P}\left(\mathbf{C}\left(0, \frac{5}{3}, e_{n}\right), \mathbb{R}^{n}\right)<\infty$, the pre-compactness theorem (Theorem 1.9) can be applied and ensures the existence of a set $F$ of finite perimeter such that, passing to a subsequence if necessary:

$$
E_{h} \cap \mathbf{C}\left(0, \frac{5}{3}, e_{n}\right) \rightarrow F
$$

note that, since $0 \in \partial E_{h} \cap \mathbf{C}\left(0, \frac{5}{3}, e_{n}\right)$, it follows (from Proposition 1.11) that $0 \in \partial F$. Besides, since $\mathbf{C}\left(0, \frac{4}{3}, e_{n}\right) \Subset \mathbf{C}\left(0, \frac{5}{3}, e_{n}\right)$, follows from the lower semi-continuity of the excess:

$$
\mathbf{e}\left(F, 0, \frac{4}{3}, e_{n}\right) \leq \liminf _{h \rightarrow \infty} \mathbf{e}\left(E_{h}, 0, \frac{4}{3}, e_{n}\right) \leq^{*}\left(\frac{3}{2}\right)^{n-1} \lim _{h \rightarrow \infty} \mathbf{e}\left(E_{h}, 0,2, e_{n}\right)=0
$$

where, in $\left(^{*}\right)$, it was used the change of scale of the excess. It then follows from Proposition 2.4 that:

$$
F \cap \mathbf{C}\left(0, \frac{4}{3}, e_{n}\right) \stackrel{\text { Lebesgue }}{\sim}\left\{y \in \mathbf{C}\left(0, \frac{4}{3}, e_{n}\right):(y-0) \cdot e_{n}=\mathbf{q} y \leq 0\right\}
$$

An infinite quantity of $E_{h}$ does not satisfy 2.6: Take a sequence $x_{h} \in \mathbf{C}\left(0,1, e_{n}\right) \cap \partial E_{h}$ such that $t_{0} \leq\left|\mathbf{q} x_{h}\right| \leq 1$. Let $x_{0} \in \overline{\mathbf{C}\left(0,1, e_{n}\right)} \cap \partial F$ the limit point of this sequence, it holds $x_{0} \in \mathbf{C}\left(0, \frac{4}{3}, e_{n}\right) \cap \partial F$ with $\left|\mathbf{q} x_{0}\right| \geq t_{0}$. On other hand, by the equivalence in 2.9 follows:

$$
\mathbf{C}\left(0, \frac{4}{3}, e_{n}\right) \cap\{x: \mathbf{q} x=0\}=\mathbf{C}\left(0, \frac{4}{3}, e_{n}\right) \cap \operatorname{spt} \mu_{\{x: \mathbf{q} x \leq 0\}}=
$$




$$
=\mathbf{C}\left(0, \frac{4}{3}, e_{n}\right) \cap \operatorname{spt} \mu_{F}=\mathbf{C}\left(0, \frac{4}{3}, e_{n}\right) \cap \partial F
$$

what contradicts the existence of $x_{0}$. So, there exists $\exists h_{0}$ such that $\forall h \geq$ $h_{0}$ the set $E_{h}$ satisfies 2.6. It follows that, for all $A^{\prime}$ Borel set of $\mathbb{R}^{n}$ (by Theorem 16.3 in [Mag12]):

$$
\begin{gathered}
\mathcal{P}\left(\mathbf{C}\left(0,1, e_{n}\right) \cap E_{h}, A^{\prime}\right)=\mathcal{P}\left(\mathbf{C}\left(0,1, e_{n}\right), E_{h}^{(1)} \cap A^{\prime}\right)+ \\
=0, \text { when } A^{\prime}=\left\{x \in \mathbf{C}\left(0,1, e_{n}\right): t_{0}<|\mathbf{q} x|<1\right\} \text { by } 2.9 \\
+\overbrace{\mathcal{P}\left(E_{h}, \mathbf{C}\left(0,1, e_{n}\right)^{(1)} \cap A^{\prime}\right)}+\mathcal{H}_{n-1}\left(\left\{\nu_{\mathbf{C}\left(0,1, e_{n}\right)}=\nu_{E_{h}}\right\} \cap A^{\prime}\right)
\end{gathered}
$$

Since

$$
\begin{gathered}
\mathcal{P}\left(\mathbf{C}\left(0,1, e_{n}\right), E_{h}^{(1)} \cap\left\{x \in \mathbf{C}\left(0,1, e_{n}\right): t_{0}<|\mathbf{q} x|<1\right\}\right)= \\
=\mathcal{H}_{n-1}\left(\partial^{*} \mathbf{C}\left(0,1, e_{n}\right) \cap E_{h}^{(1)} \cap\left\{x \in \mathbf{C}\left(0,1, e_{n}\right): t_{0}<|\mathbf{q} x|<1\right\}\right)
\end{gathered}
$$

and $\partial^{*} \mathbf{C}\left(0,1, e_{n}\right) \cap \mathbf{C}\left(0,1, e_{n}\right)=\emptyset$, we have that:

$$
\begin{gathered}
\mathcal{P}\left(\mathbf{C}\left(0,1, e_{n}\right), E_{h}^{(1)} \cap\left\{x \in \mathbf{C}\left(0,1, e_{n}\right): t_{0}<|\mathbf{q} x|<1\right\}\right)= \\
=\mathcal{H}_{n-1}\left(\left\{\nu_{\mathbf{C}\left(0,1, e_{n}\right)}=\nu_{E_{h}}\right\} \cap\left\{x \in \mathbf{C}\left(0,1, e_{n}\right): t_{0}<|\mathbf{q} x|<1\right\}\right)=0
\end{gathered}
$$

. Thus, it holds $\mathcal{P}\left(\mathbf{C}\left(0,1, e_{n}\right) \cap E_{h},\left\{x \in \mathbf{C}\left(0,1, e_{n}\right): t_{0}<|\mathbf{q} x|<1\right\}\right)=0$, what ensures $\left(^{*}\right)$ that comes in the next equation, where we set $\phi \in$ $C_{c}^{\infty}\left(\left\{x \in \mathbf{C}\left(0,1, e_{n}\right): t_{0}<|\mathbf{q} x|<1\right\}\right):$

$$
\begin{gathered}
\int 1_{\mathbf{C}\left(0,1, e_{n}\right) \cap E_{h}} \nabla \phi \mathrm{d} x=\int_{\partial^{*}\left(\mathbf{C}\left(0,1, e_{n}\right) \cap E_{h}\right)} \phi \nu_{\mathbf{C}\left(0,1, e_{n}\right) \cap E_{h}} \mathrm{~d} \mathcal{H}_{n-1}= \\
=\int \phi \mathrm{d} \mu_{\mathbf{C}\left(0,1, e_{n}\right) \cap E_{h}}={ }^{*} 0
\end{gathered}
$$

Since $\left\{x \in \mathbf{C}\left(0,1, e_{n}\right): t_{0}<|\mathbf{q} x|<1\right\}$ is a connected open set, $1_{\mathbf{C}\left(0,1, e_{n}\right) \cap E_{h}}$ is constant almost everywhere in $\left\{x \in \mathbf{C}\left(0,1, e_{n}\right): t_{0}<|\mathbf{q} x|<1\right\}$ (by Lemma 3.2 in [(au09]).

Analogously, it holds $1_{\mathbf{C}\left(0,1, e_{n}\right) \cap E_{h}}$ is equivalent to some constant in $\left\{x \in \mathbf{C}\left(0,1, e_{n}\right): t_{0}<|\mathbf{q} x|<1\right\}$. By 2.9 follows $1_{\mathbf{C}\left(0,1, e_{n}\right) \cap E_{h}}$ is equivalent to 0 , in the first case, and equivalent to 1 , in the second case. Putting all together:

$$
\begin{gathered}
|\mathbf{q} x| \leq t_{0} \quad \forall x \in \mathbf{C}\left(0,1, e_{n}\right) \cap \partial E \\
\left|\left\{x \in \mathbf{C}\left(0,1, e_{n}\right) \cap E: \mathbf{q} x>t_{0}\right\}\right|=0 \\
\left|\left\{x \in \mathbf{C}\left(0,1, e_{n}\right) \backslash E: \mathbf{q} x<-t_{0}\right\}\right|=0
\end{gathered}
$$

If exists $y \in\left\{x \in \mathbf{C}\left(0,1, e_{n}\right) \cap E: \mathbf{q} x>t_{0}\right\}$, by 2.10 , it follows that $y \in$ $\operatorname{int}(E)$, what is a contradiction with 2.11 , ensuring 2.7 . The proof of 2.8 is analogous.

From the theorem above, it was possible to show where the almost minimizing sets with bounded excess is situated or not inside a cylinder. 
As a consequence of the last result, it will be possible to prove some formulas and then define the excess measure. For this purpose, we will prove how the perimeter measure of the cylinder can be written.

Lemma 2.14. Let $r>0$ and $a<b$. We define

$$
C=\left\{\left(x^{\prime}, x_{n}\right) \in \mathbb{R}^{n}:\left|x^{\prime}\right|<r, a<x_{n}<b\right\}
$$

then:

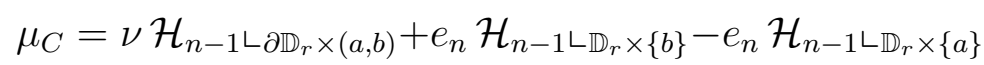

where $\nu(x)=\left(x^{\prime} / r, 0\right)$.

Proof. Firstly, we note that

$$
\begin{array}{r}
\partial C=\overbrace{\left(\partial \mathbb{D}_{r} \times\{a\}\right) \dot{U}\left(\partial \mathbb{D}_{r} \times\{b\}\right)}^{\doteq N_{0}} \dot{\cup} \\
\dot{U} \overbrace{\left(\partial \mathbb{D}_{r} \times(a, b)\right) \dot{U}\left(\mathbb{D}_{r} \times\{a\}\right) \dot{U}\left(\mathbb{D}_{r} \times\{b\}\right)}^{N^{N}}
\end{array}
$$

and

$$
\mathcal{H}_{n-1}\left(N_{0}\right)=0
$$

Since $N_{0}$ is a closed set and 2.14 holds true, we can say that $C$ has almost $C^{1}$-boundary and $N$ is the regular part of $\partial E$. From Theorem 9.6 in $[\operatorname{Mag} 12]$, for every $\phi \in C_{c}^{1}\left(\mathbb{R}^{n}\right)$,

$$
\int_{C} \nabla \phi=\int_{N} \phi \nu_{C} \mathrm{~d} \mathcal{H}_{n-1}
$$

Thus, we conclude that

$$
\mu_{C}=\nu_{C} \mathcal{H}_{n-1}\llcorner N
$$

Accordingly with the definition of $N$, we obtain that

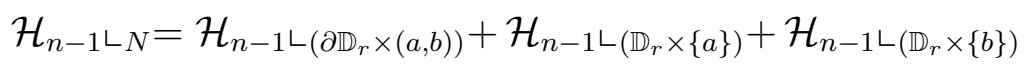

It is straightforward calculation to show that

$$
\nu_{C}\left(x^{\prime}, x_{n}\right)=\left\{\begin{array}{c}
\left(x^{\prime} / r, 0\right), \text { if } x \in \partial \mathbb{D}_{r} \times(a, b) \\
e_{n}, \text { if } x \in \mathbb{D}_{r} \times\{b\} \\
-e_{n}, \text { if } x \in \mathbb{D}_{r} \times\{a\}
\end{array}\right.
$$

Finally, by 2.15, 2.16 and the last equality, we conclude the proof.

Theorem 2.15. If $E$ is a Caccioppoli with $0 \in \partial E$ and $M \doteq C\left(0,1, e_{n}\right) \cap$ $\partial^{*} E$ such that $\exists t_{0} \in(0,1)$ satisfying:

$$
\begin{gathered}
|\boldsymbol{q} x|<t_{0} \quad \forall x \in \boldsymbol{C}\left(0,1, e_{n}\right) \cap \partial E \\
\left|\left\{x \in \boldsymbol{C}\left(0,1, e_{n}\right) \cap E: \boldsymbol{q} x>t_{0}\right\}\right|=0
\end{gathered}
$$




$$
\left|\left\{x \in \boldsymbol{C}\left(0,1, e_{n}\right) \backslash E: \boldsymbol{q} x<-t_{0}\right\}\right|=0
$$

Then, for all $G \subset \mathbb{D}_{1}$ Borel set, $\phi \in C_{c}^{0}\left(\mathbb{D}_{1}\right)$ and almost all $t \in$ $(-1,1)$, it holds:

$$
\begin{gathered}
\mathcal{H}_{n-1}(G) \leq \mathcal{H}_{n-1}\left(M \cap \boldsymbol{p}^{-1}(G)\right) \\
\mathcal{H}_{n-1}(G)=\int_{M \cap \boldsymbol{p}^{-1}(G)} \nu_{E} \cdot e_{n} \mathrm{~d} \mathcal{H}_{n-1} \\
\int_{\mathbb{D}_{1}} \phi=\int_{M} \phi(\boldsymbol{p} x) \nu_{E}(x) \cdot e_{n} \mathrm{~d} \mathcal{H}_{n-1}(x) \\
\int_{\mathbb{D}_{1} \cap E_{t}} \phi=\int_{M \cap\{\boldsymbol{q} x>t\}} \phi(\boldsymbol{p} x) \nu_{E}(x) \cdot e_{n} \mathrm{~d} \mathcal{H}_{n-1}(x)
\end{gathered}
$$

Proof. To prove 2.22 and 2.23 , it suffices to prove for all $\phi \in C_{c}^{1}\left(\mathbb{D}_{1}\right)$, since it is possible to use the Dominated Converge Theorem together with the density of $C_{c}^{1}\left(\mathbb{D}_{1}\right)$ in $C_{c}^{0}\left(\mathbb{D}_{1}\right)$. Since $\mathcal{H}_{n-1}\left\llcorner\partial^{*} E\right.$ is a Radon measure and, in particular, $\mathcal{H}_{n-1}\left(\partial^{*} E \cap \mathbf{C}\left(0,1, e_{n}\right)\right)<\infty$, it follows that, for almost all $r \in(0,1)$ :

$$
\mathcal{H}_{n-1}\left(\partial^{*} E \cap\left(\partial \mathbb{D}_{r} \times[0,1]\right)\right)=0
$$

It also holds that:

$$
\int_{\left[t_{0}, 1\right]} \int_{\mathbb{R}^{n-1}} 1_{E \cap\left(\mathbb{D}_{1} \times\{s\}\right)}(y, s) \mathrm{d} \mathcal{H}_{n-1}(y) \mathrm{d} s={ }^{*} \int_{\mathbb{R}^{n}} 1_{E \cap\left(\mathbb{D}_{1} \times\left[t_{0}, 1\right]\right)}(y) \mathrm{d} \mathcal{H}_{n} y={ }^{* *} 0
$$

where $(*)$ follows from the Fubini's Theorem and $\left({ }^{* *}\right)$ follows from 2.18. Therefore:

$$
\mathcal{H}_{n-1}\left(E \cap\left(\mathbb{D}_{1} \times\{s\}\right)\right)=0 \quad \text { for almost all } s \in\left(t_{0}, 1\right)
$$

Analogously:

$$
\mathcal{H}_{n-1}\left(E \cap\left(\mathbb{D}_{1} \times\{t\}\right)\right)=\mathcal{H}_{n-1}\left(\mathbb{D}_{1}\right) \quad \text { for almost all } \quad t \in\left(-1,-t_{0}\right)
$$

On the other hand, since $\mathcal{H}_{n-1}\left\llcorner\partial^{*} E\right.$ is a Radon measure

$$
\mathcal{H}_{n-1}\left(\partial^{*} E \cap\left(\mathbb{D}_{r} \times\{s\}\right)\right)=0
$$

holds for almost all $s \in \mathbb{R}$. Thus, fix $r \in(0,1)$ satisfying 2.24 and $s \in\left(t_{0}, 1\right)$ such that 2.25 and $\mathcal{H}_{n-1}\left(\partial^{*} E \cap\left(\mathbb{D}_{r} \times\{s\}\right)\right)=0$ are satisfied. Given $t \in(-1, s)$ such that $\mathcal{H}_{n-1}\left(\partial^{*} E \cap\left(\mathbb{D}_{r} \times\{t\}\right)\right)=0$, define $F=E \cap\left(\mathbb{D}_{r} \times(t, s)\right)$. Since the intersection of sets with finite perimeter provides a set with finite perimeter, we have that $F$ has finite perimeter. By Theorem 16.16 in [Mag12]:

$$
\mu_{F}(\cdot)=\mu_{E}\left(\left(\mathbb{D}_{r} \times(t, s)\right) \cap \cdot\right)+\mu_{\left(\mathbb{D}_{r} \times(t, s)\right)}\left(E^{(1)} \cap \cdot\right)-\overbrace{\mathcal{H}_{n-1}\left(\left\{\nu_{E}=\nu_{\mathbb{D}_{r} \times(t, s)}\right\} \cap \cdot\right)}^{=* 0}
$$


where $(*)$ follows from:

$$
\begin{gathered}
\left\{\nu_{E}=\nu_{\mathbb{D}_{r} \times(t, s)}\right\} \subset \partial^{*} E \cap \partial^{*}\left(\mathbb{D}_{r} \times(t, s)\right) \subset \\
\subset \partial^{*} E \cap\left(\partial \mathbb{D}_{r} \times[0,1] \cup \mathbb{D}_{r} \times\{t\} \cup \mathbb{D}_{r} \times\{s\}\right)
\end{gathered}
$$

together with 2.24 , the choice of $s$ and $t$. Note that $\nu(x)=\frac{\mathbf{p} x}{|\mathbf{p} x|}$ is the normal vector to the cylinder $\mathbb{D}_{1} \times \mathbb{R}$ for all $x \in \partial \mathbb{D}_{1} \times \mathbb{R}$. So, by the previous lemma, it follows:

$$
\begin{gathered}
\mu_{\left(\mathbb{D}_{r} \times(t, s)\right)}(\cdot)=e_{n} \mathcal{H}_{n-1}\left(\left(\mathbb{D}_{r} \times\{s\}\right) \cap \cdot\right)+ \\
+\nu \mathcal{H}_{n-1}\left(\left(\partial \mathbb{D}_{r} \times(t, s)\right) \cap \cdot\right)-e_{n} \mathcal{H}_{n-1}\left(\left(\mathbb{D}_{r} \times\{t\}\right) \cap \cdot\right)
\end{gathered}
$$

Keeping in mind that $\nu(x) \cdot e_{n}=0$, then, for almost all $t \in(-1, s)$ :

$$
\begin{array}{r}
e_{n} \mu_{F}(\cdot)=e_{n} \mu_{E}\left(\left(\mathbb{D}_{r} \times(t, s)\right) \cap \cdot\right)+e_{n} \mu_{\left(\mathbb{D}_{r} \times(t, s)\right)}\left(E^{(1)} \cap \cdot\right)= \\
=e_{n} \mu_{E}\left(\left(\mathbb{D}_{r} \times(t, s)\right) \cap \cdot\right)+\overbrace{\mathcal{H}_{n-1}\left(E^{(1)} \cap\left(\mathbb{D}_{r} \times\{s\}\right) \cap \cdot\right)}^{=*_{0}} \\
-\mathcal{H}_{n-1}\left(E^{(1)} \cap\left(\mathbb{D}_{r} \times\{t\}\right) \cap \cdot\right)= \\
=\left(e_{n} \cdot \nu_{E}\right) \mathcal{H}_{n-1}\left(\partial^{*} E \cap\left(\mathbb{D}_{r} \times(t, s)\right) \cap \cdot\right)-\mathcal{H}_{n-1}\left(E^{(1)} \cap\left(\mathbb{D}_{r} \times\{t\}\right) \cap \cdot\right)
\end{array}
$$

where $(*)$ follows from the choice of $s$ (satisfying 2.25) together with

$$
\left\{x \in \mathbf{C}\left(0,1, e_{n}\right) \cap E: \mathbf{q} x>t_{0}\right\}=\emptyset
$$

what is directly obtained from 2.17 and 2.18 , indeed, if exists $y$ such that

$$
y \in\left\{x \in \mathbf{C}\left(0,1, e_{n}\right) \cap E: \mathbf{q} x>t_{0}\right\}
$$

by 2.17 , it follows that $y \in \operatorname{int}(E)$, what is a contradiction with 2.18. Given $\phi \in C_{c}^{1}\left(\mathbb{D}_{1}\right)$, define the following vector field $T(x)=\phi(\mathbf{p} x) e_{n}$, $x \in \mathbb{R}^{n}$. Clearly div $T \equiv 0$, if we choose $\varphi \in C_{c}^{1}\left(\mathbb{D}_{1}\right)$ such that $\varphi(x)=$ $1, \forall x \in U$ where $U$ is an open neighborhood of $F$, we obtain that

$$
\begin{gathered}
\int e_{n} \phi \circ \mathbf{p} \mathrm{d} \mu_{F}={ }^{*} \int \varphi e_{n} \phi \circ \mathbf{p} \mathrm{d} \mu_{F}=\int \varphi T \cdot \mathrm{d} \mu_{F}= \\
=\int_{F} \operatorname{div}(\varphi T) \mathrm{d} \mathcal{H}_{n}={ }^{* *} \int_{F} \operatorname{div} T \mathrm{~d} \mathcal{H}_{n}=0
\end{gathered}
$$

where $\left(^{* *}\right)$ follows from the choice of $\varphi$, i.e. $\varphi \equiv 1$ in $\bar{F}$, which, since spt $\mu_{F} \subset \partial F$, also ensures $(*)$.

Joining this equality to 2.27 ensures that, for almost all $t \in(-1, s)$ :

$$
0=\int_{\partial^{*} E \cap\left(\mathbb{D}_{r} \times(t, s)\right)}\left(e_{n} \cdot \nu_{E}\right) \phi \circ \mathbf{p} \mathrm{d} \mathcal{H}_{n-1}-\int_{E^{(1)} \cap\left(\mathbb{D}_{r} \times\{t\}\right)} \phi \circ \mathbf{p} \mathrm{d} \mathcal{H}_{n-1}
$$

By the Dominated Convergence Theorem and taking $r, s \rightarrow 1^{-}$along 
suitable sequences follows, for almost all $t \in(-1,1)$, that:

$$
\begin{gathered}
\int_{\left(E^{(1)}\right)_{t} \cap \mathbb{D}_{1}} \phi=\int_{E^{(1)} \cap\left(\mathbb{D}_{1} \times\{t\}\right)} \phi \circ \mathbf{p} \mathrm{d} \mathcal{H}_{n-1}= \\
=\int_{\partial^{*} E \cap\left(\mathbb{D}_{r} \times(t, 1)\right)}\left(e_{n} \cdot \nu_{E}\right) \phi \circ \mathbf{p} \mathrm{d} \mathcal{H}_{n-1}= \\
=\int_{M \cap\{\mathbf{q} x>t\}}\left(e_{n} \cdot \nu_{E}\right) \phi \circ \mathbf{p} \mathrm{d} \mathcal{H}_{n-1}
\end{gathered}
$$

Since, by Fubini's Theorem, $E \stackrel{\text { Lesbegue }}{\sim} E^{(1)} \Rightarrow E_{t} \stackrel{\text { Lesbegue }}{\sim} E_{t}^{(1)}$ for almost all $t$, the proof of 2.23 is concluded. Taking $t \in\left(-1,-t_{0}\right)$ follows: $M \cap$ $\{\mathbf{q} x>t\}=M$ and, by 2.19, $\left(E^{(1)}\right)_{t} \stackrel{\text { Lesbegue }}{\sim} E_{t} \stackrel{\text { Lesbegue }}{\sim} \mathbb{D}_{1}$ and the proof of 2.22 is done. By the Cauchy-Schwarz Inequality, 2.21 implies 2.20. Lastly, we focus on the proof of 2.21. Firstly, we prove for $G^{\prime} \Subset \mathbb{D}_{1}$ Borel set. For this purpose, we denote $\varphi_{\epsilon}=\eta_{\epsilon} * 1_{G^{\prime}}$ where $\epsilon>0$ and $\eta_{\epsilon}$ is the standard mollifiers. Since $1_{G^{\prime}} \in L^{1}\left(\mathbb{D}_{1}\right)$, by Theorem 4.1 in [LCE92], we have that $\varphi \in C_{c}^{\infty}\left(\mathbb{D}_{1}\right), \varphi_{\epsilon} \rightarrow 1_{G^{\prime}} \mathcal{H}_{n-1^{-}}$almost everywhere in $\mathbb{D}_{1}$ as $\epsilon \rightarrow 0$ and

$$
\varphi_{\epsilon} \rightarrow 1_{G^{\prime}} \quad \text { in } L^{1}\left(\mathbb{D}_{1}\right)
$$

Therefore, we can apply 2.22 for $\varphi_{\epsilon}$ and ensure that

$$
\begin{array}{r}
\mathcal{H}_{n-1}\left(G^{\prime}\right)=\int_{\mathbb{D}_{1}} 1_{G^{\prime}} \mathrm{d} \mathcal{H}_{n-1}=\int_{\mathbb{D}_{1}} \lim _{n \rightarrow \infty} \varphi_{1 / n} \mathrm{~d} \mathcal{H}_{n-1}= \\
=^{*} \lim _{n \rightarrow \infty} \int_{\mathbb{D}_{1}} \varphi_{1 / n} \mathrm{~d} \mathcal{H}_{n-1}=\lim _{n \rightarrow \infty} \int_{M}\left(\nu_{E} \cdot e_{n}\right) \varphi_{1 / n} \circ \mathbf{p} \mathrm{d} \mathcal{H}_{n-1}
\end{array}
$$

where in $\left(^{*}\right)$ we used the dominated convergence theorem. Since $\varphi_{1 / n} \rightarrow$ $1_{G^{\prime}} \mathcal{H}_{n-1^{-}}$almost everywhere in $\mathbb{D}_{1}$, by the dominated convergence theorem and the convergence $\varphi_{1 / n} \circ \mathbf{p} \rightarrow 1_{G^{\prime}} \circ \mathbf{p}=1_{\mathbf{p}^{-1}\left(G^{\prime}\right)} \mathcal{H}_{n-1^{-}}$-almost everywhere in $\mathbb{D}_{1}$, we turn 2.28 into

$$
\begin{gathered}
\mathcal{H}_{n-1}\left(G^{\prime}\right)=\lim _{n \rightarrow \infty} \int_{M}\left(\nu_{E} \cdot e_{n}\right) \phi \circ \mathbf{p} \mathrm{d} \mathcal{H}_{n-1}= \\
=\int_{M}\left(\nu_{E} \cdot e_{n}\right) \lim _{n \rightarrow \infty} \varphi_{1 / n} \circ \mathbf{p} \mathrm{d} \mathcal{H}_{n-1}=\int_{M \cap \mathbf{p}^{-1}\left(G^{\prime}\right)}\left(\nu_{E} \cdot e_{n}\right) \mathrm{d} \mathcal{H}_{n-1}
\end{gathered}
$$

that is 2.21 for any Borel set compactly contained in $\mathbb{D}_{1}$. In order to conclude the proof for any Borel set $G \subset \mathbb{D}_{1}$, we take $\left\{G_{i}\right\}_{i \in \mathbb{N}}$ a sequence of Borel sets with $G_{i} \Subset \mathbb{D}_{1}, G_{i} \subset G_{i+1}$ and $G_{i} \rightarrow G$, thus

$$
\begin{gathered}
\mathcal{H}_{n-1}(G)={ }^{*} \lim _{i \rightarrow \infty} \mathcal{H}_{n-1}\left(G_{i}\right)=\lim _{i \rightarrow \infty} \int_{M \cap \mathbf{p}^{-1}\left(G_{i}\right)}\left(\nu_{E} \cdot e_{n}\right) \mathrm{d} \mathcal{H}_{n-1}= \\
=\int_{M} \lim _{i \rightarrow \infty} 1_{\mathbf{p}^{-1}\left(G_{i}\right)}\left(\nu_{E} \cdot e_{n}\right) \mathrm{d} \mathcal{H}_{n-1}=\int_{M \cap \mathbf{p}^{-1}(G)}\left(\nu_{E} \cdot e_{n}\right) \mathrm{d} \mathcal{H}_{n-1}
\end{gathered}
$$

in $\left(^{*}\right)$ we have used the continuity from below of the measure $\mathcal{H}_{n-1}$ (Theorem 1.8 in [Fol99]). 
Corollary 2.16. (Excess measure) In the conditions of the above theorem, the function

$$
\zeta: 2^{\mathbb{R}^{n-1}} \rightarrow \mathbb{R}_{+}
$$

defined by:

$$
\begin{gathered}
\zeta(G)=\mathcal{P}\left(E, C\left(0,1, e_{n}\right) \cap \boldsymbol{p}^{-1}(G)\right)-\mathcal{H}_{n-1}(G)= \\
=\mathcal{H}_{n-1}\left(M \cap \boldsymbol{p}^{-1}(G)\right)-\mathcal{H}_{n-1}(G)
\end{gathered}
$$

is a Radon measure in $\mathbb{R}^{n-1}$ concentrated in $\mathbb{D}_{1}$ and such that $\zeta\left(\mathbb{D}_{1}\right)=$ $\boldsymbol{e}\left(E, 0,1, e_{n}\right)$

Proof. The equality $\zeta\left(\mathbb{D}_{1}\right)=\mathbf{e}\left(E, 0,1, e_{n}\right)$ follows from the definition of $\zeta$, De Giorgi's Structure Theorem, i.e. $\mathcal{P}(E, \cdot)=\mathcal{H}_{n-1}\left\llcorner\partial^{*} E(\cdot)\right.$, and

$$
\mathbf{e}(E, x, r, \nu)=\frac{1}{r^{n-1}}\left(\left|\mu_{E}\right|(\mathbf{C}(x, r, \nu))-\nu \cdot \mu_{E}(\mathbf{C}(x, r, \nu))\right)
$$

Since $\mathbb{R}^{n-1}$ is locally compact Hausdorff space (LCH) and $\zeta$ is clearly a Borel measure on $\mathbb{R}^{n-1}$ finite on compact sets, by Theorem 7.8 in [Fol99], we conclude that $\zeta$ is a finite Radon measure on the Borel sets of $\mathbb{R}^{n-1}$. Then, by the Carathéodory construction, $\zeta$ induces a exterior Radon measure on $\mathbb{R}^{n-1}$.

Starting from an almost minimizing set with bounded excess at the origin, we have been building up some control of the distance, within a cylinder centered in 0 , between the topological boundary and the hyperplane defined by $e_{n}$. The control, which we have developed, assists to prove the Height Bound, which, starting from a bounded excess in a arbitrary point $x_{0}$ of the boundary, allows us to bound the distance between all points of the boundary, inside a cylinder, and $x_{0}$ by the size of the excess. In the literature, we can find the Height Bound stated in euclidean spaces of dimension bigger than 2, i.e. $n \geq 2$, in fact, the proofs, which we have checked, are not completely correct. It is due to the fact that the "Isoperimetric Inequality on Balls" is requested to be applied in dimension $n-1$, thus, it demands $n-1 \geq 2$. We are working to exhibit a proof which includes the case $n=2$. However, we have not achieved this goal at the time of writing.

Theorem 2.17. (Height Bound) Let $n \geq 3$. There exist constants $\epsilon_{0}(n), C_{0}(n)$ such that if $E$ is $\left(\Lambda, r_{0}\right)-$ minimizing set in $C\left(x_{0}, 4 r_{0}, e_{n}\right)$ with $\Lambda r_{0} \leq 1, x_{0} \in \partial E$ and

$$
\boldsymbol{e}\left(E, x_{0}, 4 r_{0}, e_{n}\right) \leq \epsilon_{0}(n)
$$

then

$\sup \left\{\frac{\left|\boldsymbol{q} y-\boldsymbol{q} x_{0}\right|}{r_{0}}: y \in \partial E \cap \boldsymbol{C}\left(x_{0}, r_{0}, e_{n}\right)\right\} \leq C_{0}(n) \boldsymbol{e}\left(E, x_{0}, 4 r_{0}, e_{n}\right)^{\frac{1}{2(n-1)}}$

Proof. Let me start with a reduction in the argument. Suppose that we have proved the theorem for $r_{0}=\frac{1}{2}$ and $x_{0}=0$. Given a set $E$ which is 
$\left(\Lambda^{\prime}, r\right)-$ minimizing set in $\mathbf{C}\left(x_{0}, 4 r, e_{n}\right)$ with $x_{0} \in \partial E, \Lambda^{\prime} r \leq 1$ and

$$
\mathbf{e}\left(E, x_{0}, 4 r, e_{n}\right) \leq \epsilon_{0}(n)
$$

By Proposition 1.3, the blow-up $E_{x_{0}, 2 r}$ of $E$ is a $\left(\Lambda, \frac{1}{2}\right)$ - minimizing set in $\mathbf{C}\left(x_{0}, 4 r, e_{n}\right)_{x_{0}, 2 r}=\mathbf{C}\left(0,2, e_{n}\right)$ with $\Lambda=\Lambda^{\prime} r, 0 \in \partial E_{x_{0}, 2 r}$. Moreover, by Proposition 2.6

$$
\mathbf{e}\left(E_{x_{0}, 2 r}, 0,2, e_{n}\right)=\mathbf{e}\left(E, x_{0}, 4 r, e_{n}\right) \leq \epsilon_{0}(n)
$$

By our reduction, since $y \in \partial E \cap \mathbf{C}\left(x_{0}, r, e_{n}\right)$ implies

$$
\frac{y-x_{0}}{2 r} \in \partial E_{x_{0}, 2 r} \cap \mathbf{C}\left(0, \frac{1}{2}, e_{n}\right)
$$

we can affirm that

$$
\begin{gathered}
\sup \left\{\frac{\left|\mathbf{q} y-\mathbf{p} x_{0}\right|}{2 r}: y \in \partial E \cap \mathbf{C}\left(x_{0}, r, e_{n}\right)\right\} \leq \\
\leq C_{0}(n) \mathbf{e}\left(E_{x_{0}, 2 r}, 0,2, e_{n}\right)^{\frac{1}{2(n-1)}}
\end{gathered}
$$

Therefore, it was possible to conclude the thesis of the Height Bound for the set $E$. So, we reduced the proof to the case with $r_{0}=\frac{1}{2}$ and $x_{0}=0$. Briefly, we want to prove the existence of $C_{0}(n)$ and $\epsilon_{0}(n)$ such that

$$
|\mathbf{q} x| \leq C_{0}(n) \mathbf{e}\left(E, 0,2, e_{n}\right)^{\frac{1}{2(n-1)}} \quad \forall x \in \partial E \cap \mathbf{C}\left(0, \frac{1}{2}, e_{n}\right)
$$

whenever $\mathbf{e}\left(E, 0,2, e_{n}\right) \leq \epsilon_{0}(n)$.

Claim 1: Exists $t_{0} \in\left(-\frac{1}{4}, \frac{1}{4}\right)$ such that, for all $x \in \mathbf{C}\left(0, \frac{1}{2}, e_{n}\right) \cap \partial E$,

$$
\left|\mathbf{q} x-t_{0}\right| \leq C(n) \mathbf{e}\left(E, 0,2, e_{n}\right)^{\frac{1}{2(n-1)}}
$$

Since $0 \in \partial E$ implies $\left|t_{0}\right| \leq C(n) \mathbf{e}\left(E, 0,2, e_{n}\right)^{\frac{1}{2(n-1)}}$, from 2.31, we obtain that

$$
|\mathbf{q} x| \leq\left|\mathbf{q} x-t_{0}\right|+\left|t_{0}\right| \leq 2 C(n) \mathbf{e}\left(E, 0,2, e_{n}\right)^{\frac{1}{2(n-1)}}
$$

To conclude, we define $C_{0}(n)=2 C(n)$.

Proof of Claim 1: First of all, assume that

$$
\epsilon_{0}(n) \leq \omega\left(n, \frac{1}{4}\right)
$$

where the constant $\omega\left(n, \frac{1}{4}\right)$ is from Theorem 2.13. If necessary, we will appropriately reduce $\epsilon_{0}(n)$. So, set $M=\partial E \cap \mathbf{C}\left(0,1, e_{n}\right)$, from 2.6

$$
|\mathbf{q} x| \leq \frac{1}{4} \quad \forall x \in M
$$

By the change of scale in the excess (Proposition 2.5) holds $\mathbf{e}\left(E, 0,1, e_{n}\right) \leq$ 
$2^{n-1} \mathbf{e}\left(E, 0,2, e_{n}\right)$, then, by the properties of the excess measure (Corollary 2.16) and the $\mathcal{H}_{n-1}$-equivalence between $\partial E \cap \mathbf{C}\left(0,2, e_{n}\right)$ and $\partial^{*} E \cap$ $\mathbf{C}\left(0,2, e_{n}\right)$ given by Corollary 1.7 , it follows that

$$
\begin{array}{r}
\mathcal{H}_{n-1}\left(\mathbf{C}\left(0,1, e_{n}\right) \cap \partial E \cap \mathbf{p}^{-1}\left(\mathbb{D}_{1}\right)\right)-\mathcal{H}_{n-1}\left(\mathbb{D}_{1}\right)= \\
=\mathcal{H}_{n-1}(M)-\mathcal{H}_{n-1}\left(\mathbb{D}_{1}\right)= \\
=\zeta\left(\mathbb{D}_{1}\right)=\mathbf{e}\left(E, 0,1, e_{n}\right) \leq 2^{n-1} \mathbf{e}\left(E, 0,2, e_{n}\right)
\end{array}
$$

As a consequence of $\zeta(G) \leq \mathbf{e}\left(E, 0,1, e_{n}\right)$, we have that

$$
\mathcal{H}_{n-1}\left(M \cap \mathbf{p}^{-1}(G)\right) \leq \mathcal{H}_{n-1}(G)+\mathbf{e}\left(E, 0,1, e_{n}\right)
$$

By a standard approximation argument and the triangle inequality, 2.23 ensures the first inequality that follows

$$
\begin{aligned}
& 0 \leq \mathcal{H}_{n-1}(M \cap\{\mathbf{q} x>t\})-\mathcal{H}_{n-1}\left(E_{t} \cap \mathbb{D}_{1}\right) \leq \\
& \leq^{*} \mathbf{e}\left(E, 0,1, e_{n}\right) \leq 2^{n-1} \mathbf{e}\left(E, 0,2, e_{n}\right)
\end{aligned}
$$

where $(*)$ follows from 2.35 taking $G=\mathbb{D}_{1}$. Note that 2.36 holds for almost all $t \in(-1,1)$. Now, we will define a function which will ensure the existence of $t_{0}$ as it is wished. So, define $f:(-1,1) \rightarrow\left[0, \mathcal{H}_{n-1}(M)\right]$ as

$$
f(t)=\mathcal{H}_{n-1}(M \cap\{\mathbf{q} x>t\})
$$

The function $f$ is right-continuous as a consequence of the continuity from below of the measure $\mathcal{H}_{n-1}\left\llcorner\partial^{*} E\right.$ (Theorem 1.8 in [Fol99]) and, evidently, $f$ is decreasing. Moreover, $f$ also satisfies

$$
\begin{array}{r}
f(t)=\mathcal{H}_{n-1}(M) \quad \forall t \in\left(-1,-\frac{1}{4}\right] \\
f(t)=0 \quad \forall t \in\left[\frac{1}{4}, 1\right)
\end{array}
$$

what follows directly from 2.33 . We now set

$$
t_{0} \doteq \inf \left\{t \in(-1,1): f(t) \leq \frac{\mathcal{H}_{n-1}(M)}{2}\right\}
$$

Note that $t_{0} \in(-1 / 4,1 / 4)$ and $t_{0}$ satisfies

$$
\begin{gathered}
f(t) \leq \frac{\mathcal{H}_{n-1}(M)}{2} \quad \forall t \in\left[t_{0}, 1\right) \\
f(t) \geq \frac{\mathcal{H}_{n-1}(M)}{2} \quad \forall t \in\left(-1, t_{0}\right)
\end{gathered}
$$

Now, we contend to prove that $t_{0}$ is the desired value by the Claim. To this end, we will divide the proof into two steps.

Step one: Suppose that

$$
f\left(t_{0}\right) \leq \sqrt{\mathbf{e}\left(E, 0,2, e_{n}\right)}
$$


Thus, if $x \in \mathbf{C}\left(0, \frac{1}{2}, e_{n}\right) \cap \partial E$ with $\mathbf{q} x>t_{0}$, by 2.33 and $\left|t_{0}\right| \leq 1 / 4$, it holds that $\mathbf{q} x-t_{0}<\frac{1}{2}$, then $\mathbf{B}\left(x, \mathbf{q} x-t_{0}\right) \Subset \mathbf{C}\left(0,1, e_{n}\right) \Subset \mathbf{C}\left(0,2, e_{n}\right)$ what allows us to use the lower density estimate in 1.12 as follows

$$
c(n) \leq \frac{\mathcal{P}\left(E, \mathbf{B}\left(x, \mathbf{q} x-t_{0}\right)\right)}{\left(\mathbf{q} x-t_{0}\right)^{n-1}} \leq \leq^{*} \frac{f\left(t_{0}\right)}{\left(\mathbf{q} x-t_{0}\right)^{n-1}}
$$

where $\left(^{*}\right)$ follows from the inclusion $\mathbf{B}\left(x, \mathbf{q} x-t_{0}\right) \subset \mathbf{C}\left(0,1, e_{n}\right) \cap\left\{\mathbf{q} x>t_{0}\right\}$ and

$$
\mathcal{P}\left(E, \mathbf{C}\left(0,1, e_{n}\right) \cap\left\{\mathbf{q} x>t_{0}\right\}\right)=\mathcal{H}_{n-1}\left(M \cap\left\{\mathbf{q} x>t_{0}\right\}\right)
$$

Therefore, by our assumption (2.39), we get that

$$
c(n)\left(\mathbf{q} x-t_{0}\right)^{n-1} \leq f\left(t_{0}\right) \leq \sqrt{\mathbf{e}\left(E, 0,2, e_{n}\right)} \quad \forall x \in \mathbf{C}\left(0, \frac{1}{2}, e_{n}\right) \cap \partial E
$$

what implies

$$
\mathbf{q} x-t_{0} \leq \frac{1}{c(n)^{\frac{1}{n-1}}} \mathbf{e}\left(E, 0,2, e_{n}\right)^{\frac{1}{2(n-1)}} \quad \forall x \in \mathbf{C}\left(0, \frac{1}{2}, e_{n}\right) \cap \partial E
$$

note that, if $\mathbf{q} x \leq t_{0}$, the inequality above (2.43) is trivial. Thus, if $f$ satisfies 2.39, the proof of the Claim 1 is done.

Step two: Suppose that

$$
f\left(t_{0}\right)>\sqrt{\mathbf{e}\left(E, 0,2, e_{n}\right)}
$$

Now, we are not able to establish the estimates in 2.40 for the constant $t_{0}$. In order to obtain an similar inequality, we shall define an auxiliary constant $t_{1}$ which will satisfy 2.40 in place of $t_{0}$. Furthermore, we will prove that $t_{1}$ and $t_{0}$ are related as follows

$$
t_{1}-t_{0} \leq C^{0}(n) \mathbf{e}\left(E, 0,2, e_{n}\right)^{\frac{1}{2(n-1)}}
$$

Therefore, we set

$$
t_{1} \doteq \inf \left\{t \in(-1,1): f(t) \leq \sqrt{\mathbf{e}\left(E, 0,2, e_{n}\right)}\right\}
$$

Thus,

$$
\begin{array}{cc}
f(t) \leq \sqrt{\mathbf{e}\left(E, 0,2, e_{n}\right)} & \forall t \in\left[t_{1}, 1\right) \\
f(t)>\sqrt{\mathbf{e}\left(E, 0,2, e_{n}\right)} & \forall t \in\left(-1, t_{1}\right)
\end{array}
$$

By our assumption (2.42) and recalling that $f$ is decreasing, we have that $t_{1}>t_{0}$. Since $f(1 / 4)=0(2.37)$, we also have $t_{0}<1 / 4$. Then, $t_{1} \in\left(t_{0}, 1 / 4\right)$. If $x \in \mathbf{C}\left(0, \frac{1}{2}, e_{n}\right) \cap \partial E$ with $\mathbf{q} x>t_{1}$, by 2.33 and the choice of $t_{1}$, it holds $\mathbf{q} x-t_{1}<\frac{1}{2}$, then

$$
\mathbf{B}\left(x, \mathbf{q} x-t_{1}\right) \Subset \mathbf{C}\left(0,1, e_{n}\right) \Subset \mathbf{C}\left(0,2, e_{n}\right)
$$


what allows to use the lower density estimate in 1.12 as follows

$$
c(n) \leq \frac{\mathcal{P}\left(E, \mathbf{B}\left(x, \mathbf{q} x-t_{1}\right)\right)}{\left(\mathbf{q} x-t_{1}\right)^{n-1}} \leq * \frac{f\left(t_{1}\right)}{\left(\mathbf{q} x-t_{1}\right)^{n-1}}
$$

where $\left(^{*}\right)$ follows from the inclusion $\mathbf{B}\left(x, \mathbf{q} x-t_{1}\right) \subset \mathbf{C}\left(0,1, e_{n}\right) \cap\left\{\mathbf{q} x>t_{1}\right\}$ and

$$
\mathcal{P}\left(E, \mathbf{C}\left(0,1, e_{n}\right) \cap\left\{\mathbf{q} x>t_{1}\right\}\right)=\mathcal{H}_{n-1}\left(M \cap\left\{\mathbf{q} x>t_{1}\right\}\right)
$$

Therefore, by the choice of $t_{1}$

$$
c(n)\left(\mathbf{q} x-t_{1}\right)^{n-1} \leq f\left(t_{1}\right) \leq \sqrt{\mathbf{e}\left(E, 0,2, e_{n}\right)} \quad \forall x \in \mathbf{C}\left(0, \frac{1}{2}, e_{n}\right) \cap \partial E
$$

what implies

$$
\mathbf{q} x-t_{1} \leq \frac{1}{c(n)^{\frac{1}{n-1}}} \mathbf{e}\left(E, 0,2, e_{n}\right)^{\frac{1}{2(n-1)}} \quad \forall x \in \mathbf{C}\left(0, \frac{1}{2}, e_{n}\right) \cap \partial E
$$

note that, when $\mathbf{q} x \leq t_{1}$ the inequality above is trivial. Now, we will prove that

$$
t_{1}-t_{0} \leq C^{0}(n) \mathbf{e}\left(E, 0,2, e_{n}\right)^{\frac{1}{2(n-1)}}
$$

To this end, by Theorem 18.11 in [Mag12], for almost all $t \in \mathbb{R}, E_{t}$ has finite perimeter and we have

$$
\mathcal{H}_{n-2}\left(\partial^{*} E_{t} \Delta\left(\partial^{*} E\right)_{t}\right)=0
$$

from this equality we find that

$$
\begin{array}{r}
\mathcal{H}_{n-2}\left(\mathbb{D}_{1} \cap \partial^{*} E_{t}\right)=\mathcal{H}_{n-2}\left(\mathbb{D}_{1} \cap\left(\partial^{*} E\right)_{t}\right)= \\
={ }^{*} \mathcal{H}_{n-2}\left(\left(\mathbf{C}\left(0,1, e_{n}\right) \cap \partial^{*} E\right)_{t}\right)
\end{array}
$$

the equality $\left(^{*}\right)$ is deduced from $\mathbb{D}_{1} \cap\left(\partial^{*} E\right)_{t}=\left(\mathbf{C}\left(0,1, e_{n}\right) \cap \partial^{*} E\right)_{t}$. From the Coarea formula for locally $(n-1)$-rectifiable (Theorem 2.93 in [LA00] taking $f=\mathbf{q}$ ), we deduce that

$$
\int_{\partial^{*} E \cap \mathbf{C}\left(0,1, e_{n}\right)} \sqrt{1-\left(\nu_{E} \cdot e_{n}\right)^{2}} \mathrm{~d} \mathcal{H}_{n-1}=\int_{\mathbb{R}} \mathcal{H}_{n-2}\left(\left(\mathbf{C}\left(0,1, e_{n}\right) \cap \partial^{*} E\right)_{t}\right) \mathrm{d} t
$$

Thus

$$
\begin{array}{r}
\int_{[-1,1]} \mathcal{H}_{n-2}\left(\mathbb{D}_{1} \cap \partial^{*} E_{t}\right) \mathrm{d} t=_{2.45}^{2.46} \int_{\mathbf{C}\left(0,1, e_{n}\right) \cap \partial^{*} E} \sqrt{1-\left(\nu_{E} \cdot e_{n}\right)^{2}} \mathrm{~d} \mathcal{H}_{n-1} \leq \\
\leq^{*} \sqrt{2} \int_{M} \sqrt{1-\nu_{E} \cdot e_{n}} \mathrm{~d} \mathcal{H}_{n-1} \leq^{* *} \sqrt{2 \mathcal{H}_{n-1}(M) \mathbf{e}\left(E, 0,1, e_{n}\right)} \leq \\
\leq^{2.35} \sqrt{2\left(\mathcal{H}_{n-1}\left(\mathbb{D}_{1}\right)+2^{n-1} \mathbf{e}\left(E, 0,2, e_{n}\right)\right)} \sqrt{2^{n-1} \mathbf{e}\left(E, 0,2, e_{n}\right)} \leq \\
\leq^{* * *} \sqrt{2^{n}\left(\mathcal{H}_{n-1}\left(\mathbb{D}_{1}\right)+2^{n-1} \epsilon_{0}(n)\right)} \sqrt{\mathbf{e}\left(E, 0,2, e_{n}\right)}
\end{array}
$$

where $\left(^{*}\right)$ follows by $(a-1)^{2} \geq 0 \Rightarrow-2 a+2 \geq-a^{2}+1$ and $M \stackrel{\mathcal{H}_{n-1}}{\sim}$ 
$\mathbf{C}\left(0,1, e_{n}\right) \cap \partial^{*} E,(* *)$ is directly from Holder's Inequality and $\left|\nu_{E}-e_{n}\right|=$ $2-2 \nu_{E} \cdot e_{n},\left({ }^{* *}\right)$ is consequence of the boundedness of the excess. Define $C^{1}(n)=\sqrt{2^{n}\left(\mathcal{H}_{n-1}\left(\mathbb{D}_{1}\right)+2^{n-1} \epsilon_{0}(n)\right)}$.

Claim 2: Exists $c^{0}(n)>0$ such that, for almost all $t \in\left[t_{0}, t_{1}\right)$

$$
\mathcal{H}_{n-2}\left(\mathbb{D}_{1} \cap \partial^{*} E_{t}\right) \geq c^{0}(n) \mathcal{H}_{n-1}\left(E_{t} \cap \mathbb{D}_{1}\right)^{\frac{n-2}{n-1}}
$$

The Claim 2 combined with 2.47 yields

$$
\int_{\left[t_{0}, t_{1}\right)} \mathcal{H}_{n-1}\left(E_{t} \cap \mathbb{D}_{1}\right)^{\frac{n-2}{n-1}} \mathrm{~d} t \leq \frac{C^{1}(n)}{c^{0}(n)} \sqrt{\mathbf{e}\left(E, 0,2, e_{n}\right)}
$$

By 2.36, for almost all $t \in\left[t_{0}, t_{1}\right)$

$$
\begin{gathered}
\mathcal{H}_{n-1}\left(E_{t} \cap \mathbb{D}_{1}\right) \geq \mathcal{H}_{n-1}(M \cap\{\mathbf{q} x>t\})-2^{n-1} \mathbf{e}\left(E, 0,2, e_{n}\right) \geq \\
\geq^{*} \sqrt{\mathbf{e}\left(E, 0,2, e_{n}\right)}-2^{n-1} \mathbf{e}\left(E, 0,2, e_{n}\right) \geq^{* *} \sqrt{\mathbf{e}\left(E, 0,2, e_{n}\right)}\left(1-2^{n-1} \sqrt{\epsilon_{0}(n)}\right)
\end{gathered}
$$

in $\left(^{*}\right)$ was used the choice of $t_{1}$ and $\left({ }^{* *}\right)$ is consequence of the boundedness of the excess. In order to have $1-2^{n-1} \sqrt{\epsilon_{0}(n)}>0$, if necessary, we will reduce the size of $\epsilon_{0}(n)$. Putting together the last two inequalities, we find that

$$
\int_{\left[t_{0}, t_{1}\right)}\left(\sqrt{\mathbf{e}\left(E, 0,2, e_{n}\right)}\left(1-2^{n-1} \sqrt{\epsilon_{0}(n)}\right)\right)^{\frac{n-2}{n-1}} \mathrm{~d} t \leq C^{1}(n) \sqrt{\mathbf{e}\left(E, 0,2, e_{n}\right)}
$$

what ensures that

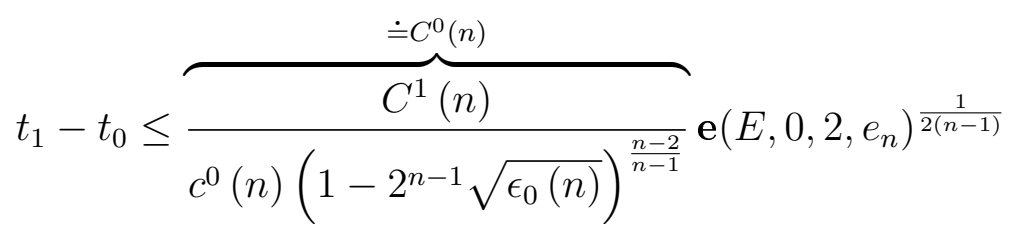

thus, by 2.43 and the last inequality

$$
\mathbf{q} x-t_{0} \leq\left(C^{0}(n)+\frac{1}{c(n)^{\frac{1}{n-1}}}\right) \mathbf{e}\left(E, 0,2, e_{n}\right)^{\frac{1}{2(n-1)}}
$$

Applying the same arguments of both Steps one and Step two for $E^{c}$, it is possible to conclude the same inequality for $t_{0}-\mathbf{q} x$, thus, we finish the proof.

Proof of Claim 2: By 2.34, 2.36 and the choice of $t_{0}$, for almost all $t \in\left[t_{0}, t_{1}\right)$, we have

$$
\begin{gathered}
\mathcal{H}_{n-1}\left(E_{t} \cap \mathbb{D}_{1}\right) \leq \mathcal{H}_{n-1}(M \cap\{\mathbf{q} x>t\})=f(t) \leq \frac{1}{2} \mathcal{H}_{n-1}(M) \leq \\
\leq \frac{\mathcal{H}_{n-1}\left(\mathbb{D}_{1}\right)+2^{n-1} \mathbf{e}\left(E, 0,2, e_{n}\right)}{2} \leq \frac{3}{4} \mathcal{H}_{n-1}\left(\mathbb{D}_{1}\right)
\end{gathered}
$$


where we reduced, if necessary, the size of $\epsilon_{0}(n)$ provided that $2^{n-2} \epsilon_{0}(n) \leq$ $\mathcal{H}_{n-1}\left(\mathbb{D}_{1}\right)$. The inequalities above allow us to use the relative isoperimetric inequality (Proposition 12.37 in [Mag12]) for $E_{t} \cap \mathbb{D}_{1}$ as a Caccioppoli in $\mathbb{R}^{n-1}$, namely

$$
\mathcal{P}\left(E_{t} \cap \mathbb{D}_{1}, \mathbb{D}_{1}\right) \geq c^{0}(n) \mathcal{H}_{n-1}\left(E_{t} \cap \mathbb{D}_{1}\right)^{\frac{n-2}{n-1}}
$$

where we used both $n-1 \geq 2$ and $\mathcal{H}_{n-1}$ coincide with the Lebesgue measure in $\mathbb{R}^{n-1}$. We recall that

$$
\mathcal{P}\left(E_{t} \cap \mathbb{D}_{1}, \mathbb{D}_{1}\right) \leq \mathcal{P}\left(\mathbb{D}_{1}, \mathbb{D}_{1}\right)+\mathcal{P}\left(E_{t}, \mathbb{D}_{1}\right)
$$

Since $\partial^{*} \mathbb{D}_{1} \cap \mathbb{D}_{1}=\emptyset$ and

$$
\mathcal{P}\left(\mathbb{D}_{1}, \mathbb{D}_{1}\right)=\mathcal{H}_{n-2}\left(\partial^{*} \mathbb{D}_{1} \cap \mathbb{D}_{1}\right)
$$

the first term on the right side of 2.50 is equal to 0. By the De Giorgi's Structure Theorem, we have that

$$
\mathcal{P}\left(E_{t}, \mathbb{D}_{1}\right)=\mathcal{H}_{n-2}\left(\partial^{*} E_{t} \cap \mathbb{D}_{1}\right)
$$

what, by 2.49, concludes the proof of the Claim. 


\section{Approximation theorems}

In this chapter, we will use the theorems that we have proved for an almost minimizing set $E$, as the Height Bound and the Small-Excess Position, to construct a Lipschitz function $u$ such that the graph of $u$ satisfies some properties. For instance, one of these properties will make it possible to locally insert the piece of the boundary of $E$ with bounded excess into the graph of $u$. Moreover, we will be able to measure, with respect to $\mathcal{H}_{n-1}$, how large the portion of the boundary of $E$ will not be contained in the graph of $u$. We shall prove that the function $u$ possess one property that will be called almost harmonicness which will allow us to approximate the function $u$ by harmonic functions and thus making it possible to prove a new estimate on the excess. Henceforth, we will use the following notations for the hypograph and epigraph of a function $u: \mathbb{R}^{n-1} \rightarrow \mathbb{R}$

$$
\begin{gathered}
\operatorname{hypo}(u)=\left\{(z, t) \in \mathbb{R}^{n}: t>u(z)\right\} \\
\operatorname{epi}(u)=\left\{(z, t) \in \mathbb{R}^{n}: t<u(z)\right\}
\end{gathered}
$$

\subsection{Lipschitz boundary criterion}

The first theorem shows that, if the regular part of the boundary of a Caccioppoli set, that is, its reduced boundary, is at least of regularity Lipschitz inside a cylinder, the topological boundary has the same regularity.

Theorem 3.1. Let $E$ be a Caccioppoli with spt $\mu_{E}=\partial E, 0 \in E$. If $u: \mathbb{R}^{n-1} \rightarrow \mathbb{R}$ is a Lipschitz function with Lip $(u) \leq 1$ and $C\left(0,1, e_{n}\right) \cap$ $\partial^{*} E \subset G(u) \cap \boldsymbol{C}\left(0,1, e_{n}\right)$, then $\boldsymbol{C}\left(0,1, e_{n}\right) \cap \partial E=\boldsymbol{C}\left(0,1, e_{n}\right) \cap G(u)$ and 
either

$$
\begin{array}{r}
C\left(0,1, e_{n}\right) \cap(E \backslash \partial E)=C\left(0,1, e_{n}\right) \cap \operatorname{epi}(u) \\
\nu_{E}(z, u(z))=\frac{-(\nabla u(z),-1)}{\sqrt{1+|\nabla u(z)|^{2}}} \quad \text { for almost all } z \in \mathbb{D}_{1}
\end{array}
$$

$\mathrm{Or}$

$$
\begin{array}{r}
C\left(0,1, e_{n}\right) \cap(E \backslash \partial E)=C\left(0,1, e_{n}\right) \cap \text { hypo }(u) \\
\nu_{E}(z, u(z))=\frac{(\nabla u(z),-1)}{\sqrt{1+|\nabla u(z)|^{2}}} \quad \text { for almost all } z \in \mathbb{D}_{1}
\end{array}
$$

Proof. Since $G(u) \cap \mathbf{C}\left(0,1, e_{n}\right)$ is closed, it holds

$$
\mathbf{C}\left(0,1, e_{n}\right) \cap \partial E=\mathbf{C}\left(0,1, e_{n}\right) \cap \overline{\partial^{*} E} \subset G(u) \cap \mathbf{C}\left(0,1, e_{n}\right)
$$

Note that $u(0)=0$, because $(0,0) \in \partial E$. Since spt $\mu_{E}=\partial E$, by 3.3 , we have that $\mu_{E}\left(\mathbf{C}\left(0,1, e_{n}\right) \cap \operatorname{epi}(u)\right)=\mu_{E}\left(\mathbf{C}\left(0,1, e_{n}\right) \cap\right.$ hypo $\left.(u)\right)=0$. Then, for all $\phi \in C_{c}^{\infty}(\operatorname{epi}(u))$

$$
\int_{\operatorname{epi}(u)} 1_{E} \nabla \phi=\int_{\operatorname{epi}(u) \cap \partial^{*} E} \phi \mathrm{d} \mu_{E}=0
$$

analogously for hypo $(u)$. By the connectedness of the epigraph and the hypograph and Lemma 3.2 in [(au09], we find that $1_{E}$ is equivalent to a constant in each one of them.

If these constants are equal, we deduce that $1_{E}$ is constant almost everywhere in $\mathbf{C}\left(0,1, e_{n}\right)$ what provides that $\left|\mu_{E}\right|\left(\mathbf{C}\left(0,1, e_{n}\right)\right)=0$. On the other hand, since $0 \in \partial E \cap \mathbf{C}\left(0,1, e_{n}\right)=\operatorname{spt} \mu_{E} \cap \mathbf{C}\left(0,1, e_{n}\right)$, we have $\left|\mu_{E}\right|\left(\mathbf{C}\left(0,1, e_{n}\right)\right)>0$ what is an absurd. If $1_{E}$ is equivalent to 1 in epi $(u)$ and equivalent to 0 in hypo $(u)$, we can show that $1_{E}$ will be truly equal these constants in each respective set. Indeed, if $\exists x \in$ epi $(u) \cap \mathbf{C}\left(0,1, e_{n}\right)$ such that $1_{E}(x)=0$, we have $x \in \partial E$. But $\mathbf{C}\left(0,1, e_{n}\right) \cap \partial E \subset G(u) \cap$ $\mathbf{C}\left(0,1, e_{n}\right)$ which is another absurd. Thus, $1_{E}$ is equal to 1 in epi $(u)$ and, analogously, it is equal to 0 in hypo $(u)$. It concludes the proof of the both first equalities in 3.1 and 3.2. We now turn our attention to the proof of the formulas of $\nu_{E}$. Since they are mostly the same, we will show the formula on 3.1. We have proved that both

$$
\mathbf{C}\left(0,1, e_{n}\right) \cap(E \backslash \partial E)=\mathbf{C}\left(0,1, e_{n}\right) \cap \text { epi }(u)
$$

is valid, thus, we obtain that $\left|(E \Delta G(u)) \cap \mathbf{C}\left(0,1, e_{n}\right)\right|=0$. From 0.1 , we find that

$$
\mu_{E \cap \mathbf{C}\left(0,1, e_{n}\right)}=\mu_{G(u) \cap \mathbf{C}\left(0,1, e_{n}\right)}
$$

Thus, since the outer unit normal of $G(u) \cap \mathbf{C}\left(0,1, e_{n}\right)$ exists almost everywhere, we have that

$$
\nu_{E}(x)=\nu_{G(u)}(x) \quad \text { for almost all } x \in \mathbf{C}\left(0,1, e_{n}\right) \cap G(u)
$$

Therefore, our problem turn into the proof of the formula for the outer 
unit normal of a Lipschitz graph. To this end, if we define $f(z)=(z, u(z))$ for all $z \in \mathbb{D}_{1}$, by Lemma 10.4 in [Mag12], we conclude that

$$
T_{f(z)} G(u)=\nabla f(z)\left(\mathbb{R}^{n-1}\right)
$$

Since $\nabla f(z)=\left(\begin{array}{c}i d_{\mathbb{R}} n-1 \\ \nabla u(z)\end{array}\right)$ for almost all $z \in \mathbb{D}_{1}$, we show that

$$
\begin{gathered}
v \in T_{f(z)} G(u) \Leftrightarrow \quad \exists w \in \mathbb{R}^{n-1}: \quad v=\nabla f(z) w=(w, w \cdot \nabla u(z)) \Leftrightarrow \\
\Leftrightarrow v \cdot(-\nabla u(z), 1)=0 \Leftrightarrow v \in(-\nabla u(z), 1)^{\perp}
\end{gathered}
$$

Thus, $\nu_{E}(z)=\frac{(-\nabla u(z), 1)}{\sqrt{1+|\nabla u(z)|^{2}}}$ for almost all $z \in \mathbb{D}_{1}$.

Corollary 3.2. In the conditions of the theorem above, given $G \subset \mathbb{D}_{1}$ Borel set, it holds

$$
\mathcal{P}\left(E, C\left(0,1, e_{n}\right) \cap \boldsymbol{p}^{-1}(G)\right)=\int_{G} \sqrt{|\nabla u(z)|^{2}+1} \mathrm{~d} z
$$

Proof. From the area formula (Theorem 3.8 in [LCE92]), we have that

$$
\mathcal{H}_{n-1}(\{(z, u(z)): z \in G\})=\int_{G} \sqrt{|\nabla u(z)|^{2}+1} \mathrm{~d} z
$$

Since $\mathbf{C}\left(0,1, e_{n}\right) \cap \partial E=\mathbf{C}\left(0,1, e_{n}\right) \cap G(u)$, we turn the last equality into

$$
\mathcal{H}_{n-1}\left\llcorner_{\partial E}\left(\mathbf{C}\left(0,1, e_{n}\right) \cap \mathbf{p}^{-1}(G)\right)=\int_{G} \sqrt{|\nabla u(z)|^{2}+1} \mathrm{~d} z\right.
$$

In the last theorem (3.1), we have established that $\nu_{E}(z, u(z))$ exists for almost all $z \in \mathbb{D}_{1}$. As a consequence of this existence and the properties of the Hausdorff measure under Lipschitz maps (Theorem 2.3 in [LA00]), we obtain that

$$
\mathcal{H}_{n-1}(f(N)) \leq \operatorname{Lip}(f) \mathcal{H}_{n-1}(N)=0
$$

where we have set $f(z)=(z, u(z))$ and $N \subset \mathbb{D}_{1}$ as the set of points where $\nu_{E}(z, u(z))$ does not exist. Note that $\left(\partial E \backslash \partial^{*} E\right) \cap \mathbf{C}\left(0,1, e_{n}\right) \subset f(N)$, therefore, $\partial E \cap \mathbf{C}\left(0,1, e_{n}\right) \stackrel{\mathcal{H}_{n-1}}{\sim} \partial^{*} E \cap \mathbf{C}\left(0,1, e_{n}\right)$ what, by 3.4, implies that

$$
\begin{gathered}
\mathcal{P}\left(E, \mathbf{C}\left(0,1, e_{n}\right) \cap \mathbf{p}^{-1}(G)\right)= \\
=\mathcal{H}_{n-1}\left\llcorner_{\partial^{*} E}\left(\mathbf{C}\left(0,1, e_{n}\right) \cap \mathbf{p}^{-1}(G)\right)=\mathcal{H}_{n-1}\llcorner\partial E\right. \\
=\int_{G} \sqrt{|\nabla u(z)|^{2}+1} \mathrm{~d} z
\end{gathered}
$$




\subsection{Lipschitzian approximation}

We have developed sufficient tools to state the first result which provides a kind of regularity to a piece of the topological boundary of an almost minimizing set. Indeed, if $x_{0} \in \partial E$ is such that $\mathbf{e}\left(E, x_{0}, 25 r, e_{n}\right)$ is bounded by a constant depending only on the dimension $n$, we will be able to show that the piece of $\partial E \cap \mathbf{C}\left(x_{0}, r, e_{n}\right)$ with bounded excess, i.e. $M_{0}$ is a subset of the graph of a Lipschitz function $\Gamma \cap \mathbf{C}\left(x_{0}, r, e_{n}\right)$. Moreover, the result will state that the size of $(\partial E \Delta \Gamma) \cap \mathbf{C}\left(x_{0}, r, e_{n}\right)$, i.e. the piece that is not contained in the Lipschitz graph $\Gamma \cap \mathbf{C}\left(x_{0}, r, e_{n}\right)$, is controlled by the size of the excess.

Theorem 3.3. (Lipschitzian Approximation) Let $n \geq 3$, there exist constants $C_{1}(n), \epsilon_{1}(n)$ and $\delta_{0}(n)$ such that if $E$ is a $\left(\Lambda, r_{0}\right)$-minimizing set in $C\left(x_{0}, 25 r, e_{n}\right)$ with $\Lambda r_{0} \leq 1, x_{0} \in \partial E$ and

$$
25 r<r_{0}, \quad \boldsymbol{e}\left(E, x_{0}, 25 r, e_{n}\right) \leq \epsilon_{1}(n)
$$

there exists a Lipschitz function $u: \mathbb{R}^{n-1} \rightarrow \mathbb{R}$ with Lip $(u)<1$ and

$$
\sup _{\mathbb{R}^{n}} \frac{|u|}{r} \leq C_{1}(n) \boldsymbol{e}\left(E, x_{0}, 25 r, e_{n}\right)^{\frac{1}{2(n-1)}}
$$

such that, if $M=C\left(x_{0}, r, e_{n}\right) \cap \partial E$ and

$$
M_{0}=\left\{y \in M: \sup _{0<s<8 r} e\left(E, y, s, e_{n}\right) \leq \delta_{0}(n)\right\}
$$

it holds

$$
M_{0} \subset M \cap \Gamma
$$

where $\Gamma=\left(x_{0}+G(u)\right) \cap \boldsymbol{C}\left(x_{0}, r, e_{n}\right)$. Moreover

$$
\frac{\mathcal{H}_{n-1}(M \Delta \Gamma)}{r^{n-1}} \leq C_{1}(n) e\left(E, x_{0}, 25 r, e_{n}\right)
$$

Proof. Suppose that we have proved the existence of constants $C_{1}(n), \epsilon_{1}(n)$ and $\delta_{0}(n)$ in the case that $E$ is a $\left(\Lambda^{\prime}, r_{0}^{\prime}\right)$-minimizing set in $\mathbf{C}\left(0,25, e_{n}\right)$ with $\Lambda^{\prime} r_{0}^{\prime} \leq 1,0 \in \partial E$ and

$$
25<r_{0}^{\prime}, \quad \mathbf{e}\left(E, 0,25, e_{n}\right) \leq \epsilon_{1}(n)
$$

Thus, if $E^{\prime}$ is in the conditions of the theorem, we have that, by Proposition 1.3, $E=E_{x_{0}, r}^{\prime}$ is a $\left(\Lambda^{\prime}, r_{0}^{\prime}\right)$ - minimizing set in $\mathbf{C}\left(0,25, e_{n}\right)$ with $\Lambda^{\prime}=\Lambda r$ and $r_{0}^{\prime}=\frac{r_{0}}{r}$. Moreover, we have that $\Lambda^{\prime} r_{0}^{\prime} \leq 1,0 \in \partial E$ and, by Proposition 2.6, $\mathbf{e}\left(E, 0,25, e_{n}\right)=\mathbf{e}\left(E^{\prime}, x_{0}, 25 r, e_{n}\right) \leq \epsilon_{1}(n)$. Therefore, by our assumption, exists $u: \mathbb{R}^{n-1} \rightarrow \mathbb{R}$ with $\operatorname{Lip}(u)<1$ with the properties 
above. Taking $u_{r}(z)=r u\left(\frac{z}{r}\right)$, we have

$\sup _{\mathbb{R}^{n}} \frac{\left|u_{r}\right|}{r}=\sup _{\mathbb{R}^{n}}|u| \leq C_{1}(n) \mathbf{e}\left(E, 0,25, e_{n}\right)^{\frac{1}{2(n-1)}}=C_{1}(n) \mathbf{e}\left(E^{\prime}, x_{0}, 25 r, e_{n}\right)^{\frac{1}{2(n-1)}}$

We set $M^{\prime} \doteq \mathbf{C}\left(x_{0}, r, e_{n}\right) \cap \partial E^{\prime}$ and

$$
M_{0}^{\prime} \doteq\left\{y \in M^{\prime}: \sup _{0<s<8 r} \mathbf{e}\left(E, y, s, e_{n}\right) \leq \delta_{0}(n)\right\}
$$

By Proposition 2.6, it is straightforward to verify that $M_{x_{0}, r}^{\prime}=M,\left(M_{0}^{\prime}\right)_{x_{0}, r}=$ $M_{0}$ and $\Gamma_{\frac{-x_{0}}{r}, \frac{1}{r}}=\left(r G(u)+x_{0}\right) \cap \mathbf{C}\left(x_{0}, r, e_{n}\right)=\left(G\left(u_{r}\right)+x_{0}\right) \cap \mathbf{C}\left(x_{0}, r, e_{n}\right) \doteq$ $\Gamma_{r}$. Then, since $M_{0} \subset M \cap \Gamma$, we deduce that $M_{0}^{\prime} \subset M^{\prime} \cap \Gamma_{\frac{-x_{0}}{r}, \frac{1}{r}}=M^{\prime} \cap \Gamma_{r}$ that is 3.6. Taking into account the properties of $\mathcal{H}_{n-1}$ with respect to translations and homotheties (Proposition 2.49 in [LA00]) and

$$
\begin{gathered}
M^{\prime} \Delta \Gamma_{r}=\left(M^{\prime} \cap \Gamma_{r}^{c}\right) \bigcup\left(M^{\prime c} \cap \Gamma_{r}\right)= \\
=\left(\left(M \cap \Gamma^{c}\right) \bigcup\left(M^{c} \cap \Gamma\right)\right)_{\frac{-x_{0}}{r}, \frac{1}{r}}=(M \Delta \Gamma)_{\frac{-x_{0}}{r}, \frac{1}{r}}
\end{gathered}
$$

we can conclude 3.7. From now on, we shall prove the theorem for $E$ being a $\left(\Lambda^{\prime}, r_{0}^{\prime}\right)-$ minimizing set in $\mathbf{C}\left(0,25, e_{n}\right)$ with $\Lambda^{\prime} r_{0}^{\prime} \leq 1,0 \in \partial E$ and

$$
25<r_{0}^{\prime}, \quad \mathbf{e}\left(E, 0,25, e_{n}\right) \leq \epsilon_{1}(n)
$$

To this end, take the constants $\epsilon_{0}(n), C_{0}(n)$ given by the Height Bound (Theorem 2.17). Assume that $\epsilon_{1}(n) \leq \epsilon_{0}(n)$ in order to apply the Height Bound for $E$ (taking $r_{0}=\frac{25}{4}$ and $\Lambda=\Lambda^{\prime}$ ). Therefore

$$
\sup \left\{|\mathbf{q} y|: y \in \partial E \cap \mathbf{C}\left(0, \frac{25}{4}, e_{n}\right)\right\} \leq \frac{25 C_{0}(n)}{4} \mathbf{e}\left(E, 0,25, e_{n}\right)^{\frac{1}{2(n-1)}}
$$

where we will still denote the constant $\frac{25 C_{0}(n)}{4}$ by $C_{0}(n)$. Recalling 2.32 where we asked that $\epsilon_{0}(n) \leq \omega\left(n, \frac{1}{4}\right)$, and, if necessary, reducing $\epsilon_{1}(n)$ in order to have $\mathbf{e}\left(E, 0,2, e_{n}\right) \leq\left(\frac{25}{2}\right)^{n-1} \mathbf{e}\left(E, 0,25, e_{n}\right) \leq \epsilon_{1}(n)$, we are able to apply the Small-excess position theorem (Theorem 2.13) which provides 2.17, 2.18 and 2.19. Thus, from Corollary 2.16, we have that

$$
\begin{aligned}
0 \leq & \mathcal{H}_{n-1}\left(M \cap \mathbf{p}^{-1}(G)\right)-\mathcal{H}_{n-1}(G) \leq \mathbf{e}\left(E, 0,1, e_{n}\right) \leq \\
& \leq 25^{n-1} \mathbf{e}\left(E, 0,25, e_{n}\right) \quad \text { for all } G \subset \mathbb{D}_{1} \text { borel set }
\end{aligned}
$$

where we used Corollary 1.7 (because, in Corollary 2.16, $M$ is defined as $\left.\mathbf{C}\left(0,1, e_{n}\right) \cap \partial^{*} E\right)$. Let us start the construction of the wished Lipschitz function, fix $y \in M_{0}, x \in M$ and let $\|v\|_{e_{n}}=\max \{|\mathbf{p} v|,|\mathbf{q} v|\}, \forall v \in \mathbb{R}^{n}$. It is straightforward to verify that $\partial E_{y,\|y-x\|_{e_{n}}}=\frac{\partial E-y}{\|y-x\|_{e_{n}}}$. Therefore, since $y \in M \subset \partial E$, we have that

$$
0 \in \partial E_{y,\|y-x\|_{e_{n}}}
$$

By Proposition 1.3, we find that $E_{y,\|y-x\|_{e_{n}}}$ is a $\left(\Lambda_{y, x}, r_{y, x}\right)-$ minimizing 
set in

$\mathbf{C}\left(-y, 25\|y-x\|_{e_{n}}^{-1}, e_{n}\right)$ with $\Lambda_{y, x} r_{y, x} \leq 1$ where $\Lambda_{y, x}=\Lambda^{\prime}\|y-x\|_{e_{n}}, r_{y, x}=$ $\frac{r_{0}^{\prime}}{\|y-x\|_{e_{n}}}$. Since $y, x \in \mathbf{C}\left(0,1, e_{n}\right)$, we have that $4\|y-x\|_{e_{n}}<8$, thus, by the definition of $M_{0}$ and Proposition 2.6

$$
\mathbf{e}\left(E_{y,\|y-x\|_{e_{n}}}, 0,4, e_{n}\right)=\mathbf{e}\left(E, y, 4\|y-x\|_{e_{n}}, e_{n}\right) \leq \delta_{0}(n)
$$

We may assume that $\delta_{0}(n) \leq \epsilon_{0}(n)$ in order to apply the Height Bound

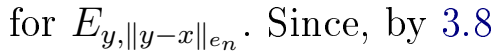

$$
r_{y, x}=\frac{r_{0}^{\prime}}{\|y-x\|_{e_{n}}}>\frac{25}{2} \text { and } \mathbf{C}\left(0,4, e_{n}\right) \subset \mathbf{C}\left(-y, \frac{25}{\|y-x\|_{e_{n}}}, e_{n}\right)
$$

we also have that $E_{y,\|y-x\|_{e_{n}}}$ is a $\left(\Lambda_{y, x}, 1\right)$ - minimizing set in $\mathbf{C}\left(0,4, e_{n}\right)$. Then, applying the Height Bound (Theorem 2.17), we find that

$$
\begin{aligned}
& \sup \left\{|\mathbf{q} v|: v \in \partial E_{y,\|y-x\|_{e_{n}}}\right.\left.\cap \mathbf{C}\left(0,1, e_{n}\right)\right\} \leq C_{0}(n) \mathbf{e}\left(E_{y,\|y-x\|_{e_{n}}}, 0,4, e_{n}\right)^{\frac{1}{2(n-1)}} \leq \\
& \leq C_{0}(n) \delta_{0}(n)^{\frac{1}{2(n-1)}}
\end{aligned}
$$

We want to apply this inequality for $\frac{x-y}{\|y-x\|_{e_{n}}}$. For this purpose, it is sufficient to recall that $x \in M=\mathbf{C}\left(0,1, e_{n}\right) \cap \partial E$. Then

$$
|\mathbf{q} y-\mathbf{q} x| \leq C_{0}(n) \delta_{0}(n)^{\frac{1}{2(n-1)}}\|y-x\|_{e_{n}}
$$

We may consider $\delta_{0}(n)<\frac{1}{C_{0}(n)^{2(n-1)}}$ in order to have $\|y-x\|_{e_{n}}=|\mathbf{p} y-\mathbf{p} x|$, by the definition of $\|\cdot\|_{e_{n}}$. Putting it all together, we conclude that

$$
|\mathbf{q} y-\mathbf{q} x| \leq \overbrace{C_{0}(n) \delta_{0}(n)^{\frac{1}{2(n-1)}}}^{\dot{\doteq} L_{n}}|\mathbf{p} y-\mathbf{p} x| \quad \text { for all } y \in M_{0}, x \in M
$$

By 3.13, we can deduce that $\mathbf{p} y=\mathbf{p} x$ implies $y=x$ for all $x, y \in M_{0}$. Then, the function $\bar{u}: \mathbf{p}\left(M_{0}\right) \rightarrow \mathbb{R}$ given by $\bar{u}=\mathbf{q} \circ \mathbf{p}^{-1}$ is well defined and also satisfy

$$
|\bar{u}(\mathbf{p} y)-\bar{u}(\mathbf{p} x)| \leq L_{n}|\mathbf{q} y-\mathbf{q} x| \quad \forall y, x \in M_{0}
$$

From Whitney-MacShane Extension Theorem (Theorem 2.3 in [J.05]), we find an extension $u: \mathbb{R}^{n} \rightarrow \mathbb{R}$ of $\bar{u}$ with $\operatorname{Lip}(u) \leq L_{n}<1$. For each $x \in M_{0}$ it holds $u(\mathbf{p} x)=\mathbf{q} x$, that is

$$
M_{0} \subset \Gamma=G(u) \cap \mathbf{C}\left(0,1, e_{n}\right)
$$

what concludes the proof of 3.6 provided we prove 3.5 , but 3.5 can be checked by 3.9 and truncating $u$. Let us prove 3.7 . To this end, by the definition of $M_{0}$, we have that

$y \in M \backslash M_{0} \Leftrightarrow y \in M$ and $\exists s_{y} \in(0,8)$ such that $\delta_{0}(n)<\mathbf{e}\left(E, y, s_{y}, e_{n}\right)$

Applying the Besicovitch's Covering Theorem (Theorem 1.27 in [LCE92]) for the family of balls $\left\{\mathrm{B}\left(y, \sqrt{2} s_{y}\right)\right\}_{y \in M \backslash M_{0}}$, we find $N_{n}$ (constant de- 
pending only of $n$ ) countable families of disjoint closed balls $\mathcal{F}_{1}, \ldots, \mathcal{F}_{N_{n}}$ such that

$$
M \backslash M_{0} \subset \bigcup_{i=1}^{N_{n}} \bigcup_{B \in \mathcal{F}_{i}} B
$$

Thus we set $k$ such that it maximizes $\sum_{B \in \mathcal{F}_{i}} \mathcal{H}_{n-1}(B)$. Denoting $\mathcal{F}_{k}$ by $\left\{\overline{\mathbf{B}\left(y_{h}, \sqrt{2} s_{h}\right)}\right\}_{h \in \mathbb{N}}$, we have that

$$
\begin{array}{r}
\mathcal{H}_{n-1}\left(M \backslash M_{0}\right) \leq \sum_{i=1}^{N_{n}} \sum_{B \in \mathcal{F}_{i}} \mathcal{H}_{n-1}(B) \leq \\
\leq N_{n} \sum_{h \in \mathbb{N}} \mathcal{H}_{n-1}\left(\overline{\mathbf{B}\left(y_{h}, \sqrt{2} s_{h}\right)}\right)
\end{array}
$$

Since $y_{h} \in \mathbf{C}\left(0,1, e_{n}\right)$ and $s_{h}<8$, we find that $\overline{\mathbf{B}\left(y_{h}, \sqrt{2} s_{h}\right)} \subset \mathbf{C}\left(0,25, e_{n}\right)$. In order to apply the density estimates (Theorem 1.5) at the inequalities below, note that $\mathbf{B}\left(y_{h},\left(1+\frac{1}{n}\right) \sqrt{2} s_{h}\right) \Subset \mathbf{C}\left(0,25, e_{n}\right)$. Recalling the $\mathcal{H}_{n-1}$-equivalence between $\partial E$ and $\partial^{*} E$, we have that

$$
\begin{gathered}
\mathcal{H}_{n-1}\left(M \backslash M_{0}\right) \leq^{3.16} N_{n} \sum_{h \in \mathbb{N}} \mathcal{H}_{n-1}\left(\overline{\mathbf{B}\left(y_{h}, \sqrt{2} s_{h}\right)}\right) \leq \\
\leq N_{n} \sum_{h \in \mathbb{N}} \mathcal{H}_{n-1}\left(\mathbf{B}\left(y_{h},\left(1+\frac{1}{n}\right) \sqrt{2} s_{h}\right)\right) \leq 3 n N_{n} \omega_{n}\left(\sqrt{2}\left(1+\frac{1}{n}\right)\right)^{n-1} \sum_{h \in \mathbb{N}} s_{h}^{n-1}
\end{gathered}
$$

By 3.15, the last inequalities and $M \backslash \Gamma \subset M \backslash M_{0}$ (from ??), we conclude that

$$
\begin{array}{r}
\mathcal{H}_{n-1}(M \backslash \Gamma) \leq \mathcal{H}_{n-1}\left(M \backslash M_{0}\right) \leq 3 n N_{n} \omega_{n}\left(\sqrt{2}\left(1+\frac{1}{n}\right)\right)^{n-1} \sum_{h \in \mathbb{N}} s_{h}^{n-1} \\
\leq \frac{3 n N_{n} \omega_{n}\left(\sqrt{2}\left(1+\frac{1}{n}\right)\right)^{n-1}}{\delta_{0}(n)} \sum_{h \in \mathbb{N}} s_{h}^{n-1} \mathbf{e}\left(E, y_{h}, s_{h}, e_{n}\right)
\end{array}
$$

Since $\mathbf{C}\left(y_{h}, s_{h}, e_{n}\right) \subset \mathbf{C}\left(0,25, e_{n}\right)$, we can state that

$$
\begin{gathered}
\sum_{h \in \mathbb{N}} s_{h}^{n-1} \mathbf{e}\left(E, y_{h}, s_{h}, e_{n}\right) \leq \sum_{h \in \mathbb{N}} \int_{\mathbf{C}\left(y_{h}, s_{h}, e_{n}\right) \cap \partial^{*} E} \frac{\left|\nu_{E}-e_{n}\right|^{2}}{2} \mathrm{~d} \mathcal{H}_{n-1} \leq \\
\leq \int_{\cup_{h \in \mathbb{N}} \mathbf{C}\left(y_{h}, s_{h}, e_{n}\right) \cap \partial^{*} E} \frac{\left|\nu_{E}-e_{n}\right|^{2}}{2} \mathrm{~d} \mathcal{H}_{n-1} \leq \int_{\mathbf{C}\left(0,25, e_{n}\right) \cap \partial^{*} E} \frac{\left|\nu_{E}-e_{n}\right|^{2}}{2} \mathrm{~d} \mathcal{H}_{n-1}
\end{gathered}
$$


Thus, by 3.17 , we can show that

$$
\mathcal{H}_{n-1}(M \backslash \Gamma) \leq \overbrace{\frac{3 n N_{n} 25^{n-1} \omega_{n}\left(\sqrt{2}\left(1+\frac{1}{n}\right)\right)^{n-1}}{\doteq^{1}(n)}} \mathbf{e}\left(E, 0,25, e_{n}\right)
$$

We can calculate the area of a graph (of codimension 1) of a Lipschitz functions (Theorem 9.1 in [Mag12]) as follows

$$
\mathcal{H}_{n-1}(\Gamma \backslash M)=\int_{\mathbf{p}(\Gamma \backslash M)} \sqrt{1+|\nabla u|^{2}} \mathrm{~d} \mathcal{H}_{n-1}
$$

Recalling that $\operatorname{Lip}(u)<1$, we can conclude that

$$
\begin{gathered}
\mathcal{H}_{n-1}(\Gamma \backslash M) \leq \sqrt{2} \mathcal{H}_{n-1}(\mathbf{p}(\Gamma \backslash M)) \leq \\
\quad \leq{ }^{3.10} \sqrt{2} \mathcal{H}_{n-1}\left(M \cap \mathbf{p}^{-1}(\mathbf{p}(\Gamma \backslash M))\right)
\end{gathered}
$$

We now notice that $x \in M \cap \mathbf{p}^{-1}(\mathbf{p}(\Gamma \backslash M)) \Rightarrow x \in M$ and $\exists z \in \Gamma \backslash M$ such that $\mathbf{p} z=\mathbf{p} x$. If we assume $x \in \Gamma$, we can write $x=(\mathbf{p} x, u(\mathbf{p} x))=$ $(\mathbf{p} z, u(\mathbf{p} z))=z$ that is a contradiction, because $z \notin M$ and $x \in M$. We have then $x \in M \backslash \Gamma$ what implies

$$
M \cap \mathbf{p}^{-1}(\mathbf{p}(\Gamma \backslash M)) \subset M \backslash \Gamma
$$

From 3.19, the last inclusion and $M \Delta \Gamma=(\Gamma \backslash M) \cup(M \backslash \Gamma)$, we conclude the proof of 3.7 as follows

$$
\begin{gathered}
\mathcal{H}_{n-1}(M \Delta \Gamma) \leq \mathcal{H}_{n-1}(\Gamma \backslash M)+\mathcal{H}_{n-1}(M \backslash \Gamma) \leq \\
\sqrt{2} \mathcal{H}_{n-1}(M \backslash \Gamma)+\mathcal{H}_{n-1}(M \backslash \Gamma) \leq^{3.18} \sqrt{2} C^{1}(n) \mathbf{e}\left(E, 0,25, e_{n}\right)
\end{gathered}
$$

The next result will state that the function $u$, from the last theorem, has interesting estimations over $\nabla u$ which will allow us to approximate $u$ by Harmonic Functions as a consequence of some results that we will show in the next section.

Proposition 3.4. (Almost harmonicness of the approximation) The function $u$ and the constant $C_{1}(n)$ in the last theorem also satisfy that $\forall \phi \in C_{c}^{1}\left(\mathbb{D}_{r}\right)$

$$
\begin{gathered}
\frac{1}{r^{n-1}} \int_{\mathbb{D}_{r}}|\nabla u|^{2} \leq C_{1}(n) \boldsymbol{e}\left(E, x_{0}, 25 r, e_{n}\right) \\
\frac{1}{r^{n-1}}\left|\int_{\mathbb{D}_{r}} \nabla u \cdot \nabla \phi\right| \leq C_{1}(n) \sup _{\mathbb{D}_{r}}|\nabla \phi|\left(\boldsymbol{e}\left(E, x_{0}, 25 r, e_{n}\right)+\Lambda r\right)
\end{gathered}
$$

Proof. We will use the same reduction that we have done in the proof of the Lipschitz Approximation Theorem (Theorem 3.3). By Theorem 4.3 in [Sim83] and the fact that $\partial^{*} E$ and $\partial E$ are $\mathcal{H}_{n-1}$-equivalent, we find 
for $\mathcal{H}_{n-1}$-almost everywhere $x \in M \cap \Gamma$ that $T_{x} \partial^{*} E=T_{x} \Gamma$ what implies

$$
\nu_{E}(x)=\lambda_{x} \frac{(-\nabla u(\mathbf{p} x), 1)}{\sqrt{1+|\nabla u(\mathbf{p} x)|^{2}}} \quad \text { with } \lambda_{x} \in\{1,-1\}
$$

Taking $\operatorname{Lip}(u)<1$ into account, we find out that

$$
\begin{gathered}
\int_{\mathbb{D}_{1}}|\nabla u|^{2} \leq \int_{\mathbf{p}(M \cap \Gamma)}|\nabla u|^{2}+\int_{\mathbf{p}(M \Delta \Gamma)}|\nabla u|^{2} \leq \\
\leq \int_{\mathbf{p}(M \cap \Gamma)} \frac{\sqrt{2}|\nabla u|^{2}}{\sqrt{1+|\nabla u|^{2}}}+\mathcal{H}_{n-1}(\mathbf{p}(M \Delta \Gamma))
\end{gathered}
$$

Since $\operatorname{Lip}(\mathbf{p})=1$ and 3.7 holds true, due to the behavior of Hausdorff measure under Lipschitz maps (Theorem 2.3 in [LCE92]), we can properly estimate $\mathcal{H}_{n-1}(\mathbf{p}(M \Delta \Gamma))$. Then, by Theorem 9.1 in [Mag12], we have that

$$
\begin{array}{r}
\int_{\mathbb{D}_{1}}|\nabla u|^{2} \leq C_{1}(n) \mathbf{e}\left(E, 0,25, e_{n}\right)+\int_{M \cap \Gamma} \frac{\sqrt{2}|\nabla u \circ \mathbf{p}|^{2}}{1+|\nabla u \circ \mathbf{p}|^{2}} \\
=C_{1}(n) \mathbf{e}\left(E, 0,25, e_{n}\right)+\int_{M \cap \Gamma} \sqrt{2}\left|\mathbf{p} \nu_{E}\right|^{2}
\end{array}
$$

Since $(a-1)^{2} \geq 0$ implies $1-a \geq \frac{1-a^{2}}{2}$ for all $a \in \mathbb{R}$, we can affirm that

$$
\frac{\left|\nu_{E}-e_{n}\right|^{2}}{2}=1-\nu_{E} \cdot e_{n} \geq \frac{1-\left(\nu_{E} \cdot e_{n}\right)^{2}}{2}={ }^{*}\left|\mathbf{p} \nu_{E}\right|^{2}
$$

where $\left(^{*}\right)$ follows from $\left|\nu_{E}\right|^{2}=\left|\mathbf{p} \nu_{E}\right|^{2}+\left|\mathbf{q} \nu_{E}\right|^{2}$. Using the last inequality in 3.23 and the change of scale in the excess (Proposition 2.5), we can conclude the proof of 3.20 as follows

$$
\begin{aligned}
\int_{\mathbb{D}_{1}}|\nabla u|^{2} & \leq C_{1}(n) \mathbf{e}\left(E, 0,25, e_{n}\right)+\int_{M \cap \Gamma} \sqrt{2} \frac{\left|\nu_{E}-e_{n}\right|^{2}}{2} \leq \\
& \leq \max \left\{C_{1}(n), 25^{n-1} \sqrt{2}\right\} \mathbf{e}\left(E, 0,25, e_{n}\right)
\end{aligned}
$$

In order to prove 3.21 , since $\frac{1}{\sqrt{1+|\nabla u|^{2}}} \leq 1$ and 3.7 is validated, we notice that it suffices to prove

$$
\left|\int_{\mathbb{D}_{1}} \frac{\nabla u \cdot \nabla \phi}{\sqrt{1+|\nabla u|^{2}}}\right| \leq C_{1}^{\prime}(n) \sup _{\mathbb{D}_{1}}|\nabla \phi|\left(\mathcal{H}_{n-1}(M \Delta \Gamma)+\Lambda r\right)
$$

for all $\phi \in C_{c}^{1}\left(\mathbb{D}_{1}\right)$. For $\mathcal{H}_{n-1}$-almost everywhere $x \in M \cap \Gamma$, notice that

$$
\left(\nu_{E} \cdot \nabla \phi\right)\left(\nu_{E} \cdot e_{n}\right)=\frac{\nabla u(\mathbf{p} x) \cdot \nabla \phi(\mathbf{p} x)}{1+\mid \nabla u\left(\left.\mathbf{p} x\right|^{2}\right.}
$$

what follows from 3.22 , we also note that we can suppose $\sup _{\mathbb{D}_{1}}|\nabla \phi|=1$ without loss of generality. Let us state a claim which will be proved later. 


\section{Claim 1:}

$$
\left|\int_{M}\left(\nu_{E} \cdot \nabla \phi\right)\left(\nu_{E} \cdot e_{n}\right)\right| \leq C^{c}(n)\left(\mathcal{H}_{n-1}(M \Delta \Gamma)+\Lambda r\right)
$$

By the connectedness of $\mathbf{p}^{-1}(z) \cap \mathbf{C}\left(0,1, e_{n}\right), \forall z \in \mathbb{D}_{1}$, together with Theorem 2.13, $\mathbf{p}(M)$ and $\mathbb{D}_{1}$ are $\mathcal{H}_{n-1}$-equivalent what ensures that

$$
\begin{gathered}
\left|\int_{\mathbb{D}_{1}} \frac{\nabla u \cdot \nabla \phi}{\sqrt{1+|\nabla u|^{2}}}\right|=\left|\int_{\mathbf{p}(M)} \frac{\nabla u \cdot \nabla \phi}{\sqrt{1+|\nabla u|^{2}}}\right| \leq \\
\leq\left|\int_{\mathbf{p}(M \backslash \Gamma)} \frac{\nabla u \cdot \nabla \phi}{\sqrt{1+|\nabla u|^{2}}}\right|+\left|\int_{\mathbf{p}(M \cap \Gamma)} \frac{\nabla u \cdot \nabla \phi}{\sqrt{1+|\nabla u|^{2}}}\right| \leq^{*} \\
\leq \mathcal{H}_{n-1}(M \backslash \Gamma)+\left|\int_{\mathbf{p}(M \cap \Gamma)} \frac{\nabla u \cdot \nabla \phi}{\sqrt{1+|\nabla u|^{2}}}\right|
\end{gathered}
$$

where in $\left(^{*}\right)$ we have used that $\left|\frac{\nabla u \cdot \nabla \phi}{\sqrt{1+|\nabla u|^{2}}}\right| \leq 1$ and the behavior of the Hausdorff measure under Lipschitz maps (Theorem 2.3 in [LCE92]). From the last inequality and Theorem 9.1 in [Mag12], we have that

$$
\left|\int_{\mathbb{D}_{1}} \frac{\nabla u \cdot \nabla \phi}{\sqrt{1+|\nabla u|^{2}}}\right| \leq \mathcal{H}_{n-1}(M \backslash \Gamma)+\left|\int_{M \cap \Gamma} \frac{\nabla u \circ \mathbf{p} \cdot \nabla \phi \circ \mathbf{p}}{1+|\nabla u \circ \mathbf{p}|^{2}}\right|
$$

Then, by 3.24 and with the help of Claim 1, we obtain that

$$
\begin{gathered}
\left|\int_{\mathbb{D}_{1}} \frac{\nabla u \cdot \nabla \phi}{\sqrt{1+|\nabla u|^{2}}}\right| \leq \mathcal{H}_{n-1}(M \backslash \Gamma)+\left|\int_{M \cap \Gamma}\left(\nu_{E} \cdot \nabla \phi\right)\left(\nu_{E} \cdot e_{n}\right)\right| \leq \\
\leq \mathcal{H}_{n-1}(M \Delta \Gamma)+C^{c}(n)\left(\mathcal{H}_{n-1}(M \Delta \Gamma)+\Lambda r\right)
\end{gathered}
$$

choosing wisely the constant depending only on the dimension $n$, we conclude the proof of the proposition. Now, we turn our attention to the proof of Claim 1.

Proof of Claim 1: We recall our assumption on $\phi$, i.e. $\sup _{\mathbb{D}_{1}}|\nabla \phi|=$ 1 , then, the Mean Value Theorem (in several variables) yields

$$
\sup _{\mathbb{D}_{1}}|\phi| \leq \sup _{\mathbb{D}_{1}}|x| \sup _{\mathbb{D}_{1}}|\nabla \phi|=1
$$

Now, we choose $\alpha \in C_{c}^{\infty}((-1,1))$ with

$$
0 \leq \alpha(s) \leq 1, \forall s \in(-1,1)
$$

and

$$
\alpha(s)=1, \forall s \in\left[-\frac{1}{4}, \frac{1}{4}\right]
$$

Then, fix $t \in\left(-\frac{1}{5}, \frac{1}{5}\right)$ and define

$$
g_{t}(s)=s+t \alpha(s), \forall s \in \mathbb{R}
$$


In order to $g_{t}$ be invertible on the real line, we require that $\left|\alpha^{\prime}(s)\right|<$ $5, \forall s \in \mathbb{R}$. Indeed, we have for each $t \in(-1 / 5,1 / 5)$ that

$$
g_{t}^{\prime}(s)=1+t \alpha^{\prime}(s)>0, \forall s \in \mathbb{R}
$$

Hence, if we define

$$
f_{t}(x)=x+t \alpha(\mathbf{q} x) \phi(\mathbf{p} x) e_{n} \quad \forall x \in \mathbb{R}^{n}
$$

and $x=\left(x^{\prime}, x_{n}\right)$, we obtain that $f_{t}\left(x^{\prime}, x_{n}\right)=\left(x^{\prime}, g_{t \phi\left(x^{\prime}\right)}\left(x_{n}\right)\right)$. For any $x^{\prime} \in \mathbb{R}^{n-1}, t \in(-1 / 5,1 / 5)$, since $t \phi\left(x^{\prime}\right) \in(-1 / 5,1 / 5)$, we have that $g_{t \phi\left(x^{\prime}\right)}$ is invertible on $\mathbb{R}$. Thus, $f_{t}$ is invertible as well and we note that

$$
J f_{t}(x)=\operatorname{det}\left(\begin{array}{c|c}
i d_{\mathbb{R}^{n-1}} & 0 \\
\hline 0 & g_{t \phi\left(x^{\prime}\right)}^{\prime}\left(x_{n}\right)
\end{array}\right)=g_{t \phi\left(x^{\prime}\right)}^{\prime}\left(x_{n}\right)>0
$$

Then, $\left\{f_{t}\right\}_{t \in\left(-\frac{1}{5}, \frac{1}{5}\right)}$ is a one-parameter family of diffeomorphisms. In order to prove that $f_{t}(E)$ is a competitor for the almost minimality of $E$, we remind that, since $f_{t}$ is a diffeomorphism, $f_{t}(E)$ is a set of finite perimeter and $f_{t}\left(\partial^{*} E\right), \partial^{*} f_{t}(E)$ are $\mathcal{H}_{n-1}$-equivalent (Proposition 17.1 in [Mag12]). Denote by supp the support of a function (i.e. $\operatorname{supp} f=$ $\overline{\{x \in \operatorname{dom}(f): f(x) \neq 0\}})$. By the definition of $f_{t}, \operatorname{supp} \alpha \Subset(-1,1)$ and $\operatorname{supp} \phi \Subset \mathbb{D}_{1}$, we find that

$$
\operatorname{supp}\left(f_{t}-i d_{\mathbb{R}^{n}}\right) \subset \operatorname{supp}(\alpha \circ \mathbf{q}) \cap \operatorname{supp}(\phi \circ \mathbf{p}) \Subset \mathbf{C}\left(0,1, e_{n}\right)
$$

whenever $t \in\left(-\frac{1}{5}, \frac{1}{5}\right)$. Then, we conclude that

$$
f_{t}(E) \Delta E \subset \operatorname{supp}\left(f_{t}-i d_{\mathbb{R}^{n}}\right) \Subset \mathbf{C}\left(0,1, e_{n}\right)
$$

Therefore, using the almost minimality condition of $E$, for all $\forall t \in$ $\left(-\frac{1}{5}, \frac{1}{5}\right)$,

$$
\mathcal{P}\left(E, \mathbf{C}\left(0,1, e_{n}\right)\right) \leq \mathcal{P}\left(f_{t}(E), \mathbf{C}\left(0,1, e_{n}\right)\right)+\Lambda^{\prime}\left|f_{t}(E) \Delta E\right|
$$

From Lemma 17.9 in [Mag12], 3.25 and the compactness of $\operatorname{supp}(\alpha \circ \mathbf{q}) \cap$ $\operatorname{supp}(\phi \circ \mathbf{p})$, we obtain the existence of constants $K(n)$ and $\epsilon_{0}<\frac{1}{5}$ such that, for all $t \in\left(-\epsilon_{0}, \epsilon_{0}\right)$,

$$
\begin{aligned}
&\left|f_{t}(E) \Delta E\right| \leq K(n)|t| \mathcal{P}(E, \operatorname{supp}(\alpha \circ \mathbf{q}) \cap \operatorname{supp}(\phi \circ \mathbf{p})) \leq \\
& \leq K(n)|t| \mathcal{P}\left(E, \mathbf{C}\left(0,1, e_{n}\right)\right)
\end{aligned}
$$

what, along with 3.26 , provides

$$
\begin{aligned}
& \mathcal{P}\left(E, \mathbf{C}\left(0,1, e_{n}\right)\right) \leq \mathcal{P}\left(f_{t}(E), \mathbf{C}\left(0,1, e_{n}\right)\right)+ \\
+ & \Lambda^{\prime} K(n)|t| \mathcal{P}\left(E, \mathbf{C}\left(0,1, e_{n}\right)\right) \quad \forall t \in\left(-\epsilon_{0}, \epsilon_{0}\right)
\end{aligned}
$$

Putting into account $f_{t}\left(\partial^{*} E\right) \stackrel{\mathcal{H}_{n-1}}{\sim} \partial^{*} f_{t}(E), M \stackrel{\mathcal{H}_{n-1}}{\sim} \partial^{*} E \cap \mathbf{C}\left(0,2, e_{n}\right)$ and $f_{t}\left(\mathbf{C}\left(0,1, e_{n}\right)\right)=\mathbf{C}\left(0,1, e_{n}\right)$ (because of 3.25$)$, we get that

$$
\mathcal{P}\left(f_{t}(E), \mathbf{C}\left(0,1, e_{n}\right)\right)-\mathcal{P}\left(E, \mathbf{C}\left(0,1, e_{n}\right)\right)=
$$




$$
\begin{gathered}
=\mathcal{H}_{n-1}\left(\partial^{*} f_{t}(E) \cap \mathbf{C}\left(0,1, e_{n}\right)\right)-\mathcal{H}_{n-1}\left(\partial^{*} E \cap \mathbf{C}\left(0,1, e_{n}\right)\right)= \\
=\mathcal{H}_{n-1}\left(f_{t}\left(\partial^{*} E \cap \mathbf{C}\left(0,1, e_{n}\right)\right)\right)-\mathcal{H}_{n-1}(M)
\end{gathered}
$$

By the area formula for countably $(n-1)$-rectifiable sets (Theorem 11.6 in [Mag12]), we find that

$$
\begin{array}{r}
\mathcal{P}\left(f_{t}(E), \mathbf{C}\left(0,1, e_{n}\right)\right)-\mathcal{P}\left(E, \mathbf{C}\left(0,1, e_{n}\right)\right)= \\
=\int_{\partial^{*} E \cap \mathbf{C}\left(0,1, e_{n}\right)} J^{M} f_{t} \mathrm{~d} \mathcal{H}_{n-1}-\mathcal{H}_{n-1}(M)= \\
=\int_{M}\left(J^{M} f_{t}-1\right) \mathrm{d} \mathcal{H}_{n-1}
\end{array}
$$

whenever $t \in\left(-\frac{1}{5}, \frac{1}{5}\right)$. Since $\alpha(s)=1, \forall s \in\left[-\frac{1}{4}, \frac{1}{4}\right]$ and $\mathbf{q} x<\frac{1}{4}, \forall x \in M$ (from 2.6), we obtain that

$$
f_{t}(x)=x+t \phi(\mathbf{p}(x)) e_{n}
$$

It is straightforward to verify that, for all $v=\left(v^{\prime}, v_{n}\right) \in \mathbb{R}^{n}$,

$$
\begin{gathered}
D\left(f_{t}\right)_{x} v=i d_{\mathbb{R}^{n}} v+t\left(v^{\prime} \cdot \nabla \phi \circ \mathbf{p}(x)\right) e_{n}= \\
=i d_{\mathbb{R}^{n}} v+t\left(e_{n} \otimes \nabla \phi \circ \mathbf{p}(x)\right) v \quad \text { whenever } x \in M
\end{gathered}
$$

Since $0 \neq|\nabla \phi \circ \mathbf{p}(x)| \leq 1$ and $|t|<1 / 5$, we have $|t|<|\nabla \phi \circ \mathbf{p}(x)|^{-1}$. Thus, if $\mathbf{p}_{1}$ denotes the orthogonal projection onto $\left(\nu_{E}\right)^{\perp}$, i.e.

$$
\mathbf{p}_{1}(\nabla \phi \circ \mathbf{p})=\nabla \phi \circ \mathbf{p}-\left(\nabla \phi \circ \mathbf{p} \cdot \nu_{E}\right) \nu_{E}
$$

by Lemma 23.10 in [Mag12], we find out that

$$
\begin{gathered}
J^{M} f_{t}(x)-1=J^{\left(\nu_{E}(x)\right)^{\perp}}\left(i d_{\mathbb{R}^{n}}(x)+t e_{n} \otimes \phi \circ \mathbf{p}(x)\right)-1 \leq \\
\leq t \mathbf{p}_{1}(\nabla \phi \circ \mathbf{p}(x)) \cdot e_{n}+L(n)|t \nabla \phi \circ \mathbf{p}(x)|^{2}
\end{gathered}
$$

for $\mathcal{H}_{n-1}$-a.e. $x \in M$. Note that, if $|\nabla \phi \circ \mathbf{p}(x)|=0$, the inequality above is trivially verified because of that $J^{M} f_{t}(x)=1$. Therefore, since $\sup _{\mathbb{D}_{1}}|\nabla \phi|=1$ and $e_{n} \cdot \nabla \phi \circ \mathbf{p}=0$, we have that

$$
J^{M} f_{t}-1 \leq-t\left(\nu_{E} \cdot e_{n}\right)\left(\nu_{E} \cdot \nabla \phi\right)+L(n) t^{2}
$$

for $\mathcal{H}_{n-1}$-a.e. $x \in M$. The last inequality and 3.28 ensure that

$$
\begin{gathered}
\mathcal{P}\left(f_{t}(E), \mathbf{C}\left(0,1, e_{n}\right)\right)-\mathcal{P}\left(E, \mathbf{C}\left(0,1, e_{n}\right)\right) \leq \\
\leq-t \int_{M}\left(\nu_{E} \cdot e_{n}\right)\left(\nu_{E} \cdot \nabla \phi\right) \mathrm{d} \mathcal{H}_{n-1}+L(n) t^{2} \mathcal{H}_{n-1}(M)
\end{gathered}
$$

We can find a upper bound $L^{\prime}(n)$ of $\mathcal{H}_{n-1}(M)$ by the density estimates (Corollary 1.8), then

$\mathcal{P}\left(f_{t}(E), \mathbf{C}\left(0,1, e_{n}\right)\right)-\mathcal{P}\left(E, \mathbf{C}\left(0,1, e_{n}\right)\right) \leq-t \int_{M}\left(\nu_{E} \cdot e_{n}\right)\left(\nu_{E} \cdot \nabla \phi\right) \mathrm{d} \mathcal{H}_{n-1}+L^{1}(n) t^{2}$ 
Finally, we put this inequality into 3.27 to produce, for all $t \in\left(-\epsilon_{0}, \epsilon_{0}\right)$,

$$
\begin{aligned}
\mathcal{P}\left(E, \mathbf{C}\left(0,1, e_{n}\right)\right) & \leq \mathcal{P}\left(E, \mathbf{C}\left(0,1, e_{n}\right)\right)-t \int_{M}\left(\nu_{E} \cdot e_{n}\right)\left(\nu_{E} \cdot \nabla \phi\right) \mathrm{d} \mathcal{H}_{n-1}+ \\
& +L^{1}(n) t^{2}+\Lambda^{\prime} K(n)|t| \mathcal{P}\left(E, \mathbf{C}\left(0,1, e_{n}\right)\right)
\end{aligned}
$$

We have already noticed that $\mathcal{H}_{n-1}(M)=\mathcal{H}_{n-1}\left(\partial^{*} E \cap \mathbf{C}\left(0,1, e_{n}\right)\right)=$ $\mathcal{P}\left(E, \mathbf{C}\left(0,1, e_{n}\right)\right)$ what guarantee that $L^{\prime}(n)$ also bounds $\mathcal{P}\left(E, \mathbf{C}\left(0,1, e_{n}\right)\right)$, thus

$$
\begin{gathered}
t \int_{M}\left(\nu_{E} \cdot e_{n}\right)\left(\nu_{E} \cdot \nabla \phi\right) \mathrm{d} \mathcal{H}_{n-1} \leq L^{1}(n) t^{2}+\Lambda^{\prime} K(n) L^{\prime}(n)|t| \\
\leq \overbrace{\max \left\{L^{1}(n), K(n) L^{\prime}(n)\right\}}^{\doteq C^{c}(n)}\left(t^{2}+\Lambda^{\prime}|t|\right)
\end{gathered}
$$

since it holds for all $t \in\left(-\epsilon_{0}, \epsilon_{0}\right)$, we have that

$$
|t|\left|\int_{M}\left(\nu_{E} \cdot e_{n}\right)\left(\nu_{E} \cdot \nabla \phi\right) \mathrm{d} \mathcal{H}_{n-1}\right| \leq C^{c}(n)\left(t^{2}+\Lambda^{\prime}|t|\right)
$$

Dividing by $|t|$ on both sides and taking

$$
0<|t|<\min \left\{\epsilon_{0}, \mathcal{H}_{n-1}(M \Delta \Gamma)+\Lambda^{\prime}\right\}
$$

we conclude the proof of the Claim.

\subsection{Approximations results on harmonic func- tions}

We will gather two technical results about Harmonic Functions that will be used together with the idea presented in the last chapter about the almost harmonicness of the Lipschitz approximation. First of all, let us remember the mean value property of harmonic functions, that is, $v$ harmonic function on $\mathbf{B}(0,1) \subset \mathbb{R}^{n}$, then

$$
v(x)=\underset{\partial \mathbf{B}(x, r)}{f} v \mathrm{~d} \mathcal{H}_{n-1}=\underset{\mathbf{B}(x, r)}{f} v, \quad \forall \mathbf{B}(x, r) \Subset \mathbf{B}(0,1)
$$

the proof for this result can be found in [Fol95] (Theorem 2.8 and Corollary 2.9) . 
Lemma 3.5. If $v$ is a harmonic function in $\boldsymbol{B}(0,1)$ and $w(x)=v(0)+$ $x \cdot \nabla v(0), \forall x \in \boldsymbol{B}(0,1)$, then

$$
\sup _{\boldsymbol{B}\left(0, \frac{1}{2}\right)}|\nabla v| \leq H(n)\|v\|_{L^{2}(\boldsymbol{B}(0,1))}
$$

and $\forall \alpha \in\left(0, \frac{1}{2}\right]$

$$
\sup _{\boldsymbol{B}(0, \alpha)}|v-w|^{2} \leq H(n) \alpha^{2}\|\nabla v\|_{L^{2}(\boldsymbol{B}(0,1))}
$$

Proof. Let $v$ be harmonic in $\mathbf{B}(0,1), x \in \mathbf{B}\left(0, \frac{1}{2}\right)$ and $\eta \in \mathbb{S}^{n-1}$. We claim that $\eta \cdot \nabla v$ is harmonic in $\mathbf{B}(0,1)$, indeed

$$
\begin{gathered}
\operatorname{div}(\nabla(\eta \cdot \nabla v))=\operatorname{div}\left(\left(\partial_{1}(\eta \cdot \nabla v), \ldots, \partial_{n}(\eta \cdot \nabla v)\right)\right)= \\
=\sum_{i=1}^{n} \partial_{i}^{2}(\eta \cdot \nabla v)=\sum_{i, j=1}^{n} \eta_{j} \partial_{j} \partial_{i}^{2} v= \\
=\sum_{j=1}^{n} \eta_{j} \partial_{j}(\Delta v) \equiv 0
\end{gathered}
$$

We are now able to apply the mean value property for $\eta \cdot \nabla v(3.29)$, thus

$$
\begin{aligned}
& |\eta \cdot \nabla v(x)|=\frac{1}{r^{n} \omega_{n}}\left|\int_{\mathbf{B}(0, r)} \eta \cdot \nabla v\right|=\frac{1}{r^{n} \omega_{n}}\left|\eta \cdot \int_{\mathbf{B}(0, r)} \nabla v\right|= \\
& ={ }^{*} \frac{1}{r^{n} \omega_{n}}\left|\eta \cdot \int_{\partial \mathbf{B}(0, r)} v \nu_{\mathbb{S}^{n-1}}\right|=\frac{1}{r^{n} \omega_{n}}\left|\int_{\partial \mathbf{B}(0, r)} v \eta \cdot \nu_{\mathbb{S}^{n-1}}\right| \leq \\
& \leq \frac{1}{r^{n} \omega_{n}} \int_{\partial \mathbf{B}(0, r)}\left|v \eta \cdot \nu_{\mathbb{S}^{n-1}}\right| \leq \frac{1}{r^{n} \omega_{n}} \int_{\partial \mathbf{B}(0, r)}|v(y)| \mathcal{H}_{n-1}(y)
\end{aligned}
$$

whenever $r<\frac{1}{4}$ what put us in position to apply in $\left(^{*}\right)$ the divergence theorem (Theorem 0.4 in [Fol95]). Applying the mean value property on the last inequality ensures that

$$
\begin{gathered}
|\eta \cdot \nabla v(x)| \leq \frac{1}{r^{n} \omega_{n}} \int_{\partial \mathbf{B}(0, r)}\left|\underset{\mathbf{B}(y, r)}{f} v(z) \mathcal{H}_{n-1}(z)\right| \mathcal{H}_{n-1}(y) \leq \\
\leq \frac{n \omega_{n} r^{n-1}}{\omega_{n}^{2} r^{2 n}} \int_{\mathbf{B}(x, 2 r)}|v(z)| \mathcal{H}_{n-1}(z) \leq^{*} H^{1}(n)\|v\|_{L^{2}(\mathbf{B}(0,1))}
\end{gathered}
$$

where in $\left(^{*}\right)$ we have used the Holder's inequality (6.2 in [Fol99, p. 174]). To conclude the proof of 3.30 , it is suffices to set $\eta=\frac{\nabla v}{|\nabla v|}$. As we have noticed above, $\eta \cdot \nabla v$ is harmonic on $\mathbf{B}(0,1)$, then we can apply 3.30 leading to

$$
\sup _{\mathbf{B}\left(0, \frac{1}{2}\right)}\left|\nabla^{2} v\right| \leq H^{1}(n)\|\nabla v\|_{L^{2}(\mathbf{B}(0,1))}
$$

By Taylor's Theorem with Lagrange Remainder, we can find, for all $x \in$ 
$\mathbf{B}(0, \alpha)$, a number $t \in(0,1)$ such that

$$
|v(x)-w(x)| \leq C\left|\nabla^{2} v(t x)\right||x|^{2}
$$

By the last two inequalities, the proof is concluded.

Let $U \subset \mathbb{R}^{n}$ be a open set, then we will denote the Sobolev space by $W^{1,2}(U)$ as in Definition 4.2 in [LCE92].

Lemma 3.6. For every $\tau>0$ exists $\sigma(\tau)>0$ such that if $u \in W^{1,2}(\boldsymbol{B}(0,1))$ satisfy both $\|u\|_{L^{2}(\boldsymbol{B}(0,1))} \leq 1$ and

$$
\left|\int_{\boldsymbol{B}(0,1)} \nabla u \cdot \nabla \phi\right| \leq \sigma(\tau) \sup _{\boldsymbol{B}(0,1)}|\nabla \phi| \quad \forall \phi \in C_{c}^{\infty}(\boldsymbol{B}(0,1))
$$

there exists $v$ harmonic function on $\boldsymbol{B}(0,1)$ such that $\|\nabla v\|_{L^{2}(\boldsymbol{B}(0,1))} \leq 1$ and

$$
\int_{B(0,1)}|v-u|^{2} \leq \tau
$$

Proof. By contradiction, suppose that exist $\tau>0$ and a sequence $\left\{u_{h}\right\}_{h \in \mathbb{N}} \subset$ $W^{1,2}(\mathbf{B}(0,1))$ in the conditions above with $\sigma_{h}(\tau)=\frac{1}{h}$ such that for every $v$ harmonic function with $\|\nabla v\|_{L^{2}(\mathbf{B}(0,1))} \leq 1$ it holds that

$$
\int_{\mathbf{B}(0,1)}\left|u_{h}-v\right|^{2}>\tau>0
$$

From the classical Poincaré inequality (Theorem 4.9 in [LCE92]), we have that

$$
\left\|u_{h}-\underset{\mathbf{B}(0,1)}{f} u_{h}\right\|_{L^{2^{*}}(\mathbf{B}(0,1))} \leq \overbrace{\omega_{n}^{\frac{1}{2^{*}}-\frac{1}{2}} C_{2}(n)}^{\doteq C_{2}^{\prime}(n)}\left\|\nabla u_{h}\right\|_{L^{2}(\mathbf{B}(0,1))}
$$

Since $\mathbf{B}(0,1)$ has finite $\mathcal{H}_{n}$ measure and $2<2^{*}$, we have that $\|\cdot\|_{L^{2}(\mathbf{B}(0,1))} \leq$ $\|\cdot\|_{L^{2^{*}}(\mathbf{B}(0,1))}$. Then we can conclude that

$$
\left\|u_{h}-\underset{\mathbf{B}(0,1)}{f} u_{h}\right\|_{L^{2}(\mathbf{B}(0,1))} \leq C_{2}^{\prime}(n)\left\|\nabla u_{h}\right\|_{L^{2}(\mathbf{B}(0,1))} \leq^{*} C_{2}^{\prime}(n)
$$

where $\left(^{*}\right)$ follows from our assumptions on the $L^{2}(\mathbf{B}(0,1))$-norm of $u_{h}$. Since $\left\|\nabla u_{h}\right\|_{L^{2}(\mathbf{B}(0,2))} \leq 1$ and

$$
\nabla\left(u_{h}-\underset{\mathbf{B}(0,1)}{f} u_{h}\right)=\nabla u_{h}
$$

we find that $\left\{u_{h}-f_{\mathbf{B}(0,1)} u_{h}\right\}_{h \in \mathbb{N}}$ is bounded in $W^{1,2}(\mathbf{B}(0,1))$. From the compactness of the inclusion (Theorem 4.11 in [LCE92])

$$
W^{1,2}(\mathbf{B}(0,1)) \hookrightarrow L^{2}(\mathbf{B}(0,1))
$$

we can extract a subsequence of

$$
u_{h}-\underset{\mathbf{B}(0,1)}{f} u_{h}
$$


which converges, in the $L^{2}(\mathbf{B}(0,1))$ sense, to a function $u \in L^{2}(\mathbf{B}(0,1))$. Since $\left\|\nabla u_{h}\right\|_{L^{2}(\mathbf{B}(0,2))} \leq 1$ for all $h \in \mathbb{N}$, i.e. $\left\{\nabla u_{h}\right\}_{h \in \mathbb{N}}$ is bounded in $L^{2}(\mathbf{B}(0,1))$, we can apply the Banach-Alaoglu Theorem (Theorem 3.17 in [Rud91]) and thus, up to extract a subsequence, we find $v \in L^{2}(\mathbf{B}(0,1))$ such that $\nabla u_{h}$ convergences in the weak-topology of $L^{2}(\mathbf{B}(0,1))$ to $v$. Since the weak-convergence directly implies the convergence in the distributional sense, we have that $\nabla u=v$ and then $u \in W^{1,2}(\mathbf{B}(0,1))$. We also have that the norm is lower-semicontinuous, then

$$
\|\nabla u\|_{L^{2}(\mathbf{B}(0,1))} \leq \liminf _{h \rightarrow \infty}\left\|\nabla u_{h}\right\|_{L^{2}(\mathbf{B}(0,1))} \leq 1
$$

We want to prove that $-u+f_{\mathbf{B}(0,1)} u_{h}$ is harmonic, thereby obtaining a contradiction from $u_{h}-f_{\mathbf{B}(0,1)} u_{h} \rightarrow u$ in $L^{2}(\mathbf{B}(0,1))$ and 3.32. Since $f_{\mathbf{B}(0,1)} u_{h}$ is constant, it is sufficient to prove that $u$ is harmonic. To this end, for all $\phi \in C_{c}^{\infty}(\mathbf{B}(0,1))$ note that

$$
\begin{aligned}
\left|\int_{\mathbf{B}(0,1)} \nabla u \cdot \nabla \phi\right| \leq & \left|\int_{\mathbf{B}(0,1)} \nabla u \cdot \nabla \phi-\int_{\mathbf{B}(0,1)} \nabla\left(u_{h}-\underset{\mathbf{B}(0,1)}{f} u_{h}\right) \cdot \nabla \phi\right|+ \\
& +\left|\int_{\mathbf{B}(0,1)} \nabla\left(u_{h}-\underset{\mathbf{B}(0,1)}{f} u_{h}\right) \cdot \nabla \phi\right|
\end{aligned}
$$

By 3.31 and 3.34, we get that $\left|\int_{\mathbf{B}(0,1)} \nabla\left(u_{h}-f_{\mathbf{B}(0,1)} u_{h}\right) \cdot \nabla \phi\right|$ is less or equal than $\frac{1}{h} \sup _{\mathbf{B}(0,1)}|\nabla \phi|$. We recall that

$$
\int_{\mathbf{B}(0,1)} \nabla\left(u-\left(u_{h}-\underset{\mathbf{B}(0,1)}{f} u_{h}\right)\right) \cdot \nabla \phi=\int_{\mathbf{B}(0,1)}\left(u-\left(u_{h}-\underset{\mathbf{B}(0,1)}{f} u_{h}\right)\right) \Delta \phi
$$

whenever $\phi \in C_{c}^{\infty}(\mathbf{B}(0,1))$. Then, putting the last equations into account, we conclude that

$$
\left|\int_{\mathbf{B}(0,1)} \nabla u \cdot \nabla \phi\right| \leq\left|\int_{\mathbf{B}(0,1)}\left(u-\left(u_{h}-\underset{\mathbf{B}(0,1)}{f} u_{h}\right)\right) \cdot \nabla \phi\right|+\frac{1}{h} \sup _{\mathbf{B}(0,1)}|\nabla \phi|
$$

By $u_{h}-f_{\mathbf{B}(0,1)} u_{h} \rightarrow u$ in $L^{2}(\mathbf{B}(0,1))$, the Holder's inequality (6.2 in [Fol99, p. 174]) and letting $h \rightarrow \infty$ in the last inequality, we find that $\left|\int_{\mathbf{B}(0,1)} \nabla u \cdot \nabla \phi\right|=0$ for any $\phi \in C_{c}^{\infty}(\mathbf{B}(0,1))$. Thus, we have proved that $u$ is harmonic what is sufficient to conclude the proof of the lemma as noticed before. 


\section{Regularity theory}

We now aim to refine some estimates on the excess of an almost minimizing set. The reverse Poincaré inequality will be required to the refinements that we intend to do, because of that, we shall enunciate it. Although, the proof of reverse Poincaré inequality will not be done in this work.

The main theorem of this chapter is, of course, the $C^{1, \gamma}$-regularity theorem which states, for each $\gamma \in(0,1 / 2)$, that the boundary of an almost minimizing set which satisfies a boundedness condition on the excess is, in fact, the graph of a function $u \in C^{1, \gamma}$. To prove the $C^{1, \gamma}$-regularity theorem, we will state two results on the excess which improves what we have done in Chapter 2. Furthermore, this results will allow us to show that $M_{0}$ is equal to $M$ with $M$ and $M_{0}$ as they were defined in the Lipschitz Approximation (Theorem 3.3) and hence equal to the graph of a Lipschitz function $u$. The $C^{1, \gamma}$-regularity of $u$ will outcome of the Excess Improvement (Theorem 4.4).

\subsection{Reverse Poincaré inequality}

Let us introduce another concept similar with the excess that we will call by flatness. The cylindrical flatness of a Caccioppoli set at $x \in \mathbb{R}^{n}$ with respect to $\nu \in \mathbb{S}^{n-1}$ at the scale $r>0$ is given by

$$
\mathbf{f}(E, x, r, \nu)=\inf _{c \in \mathbb{R}} \frac{1}{r^{n-1}} \int_{\mathbf{C}(x, r, \nu) \cap \partial^{*} E} \frac{|(y-x) \cdot \nu-c|}{r^{2}} \mathrm{~d} \mathcal{H}_{n-1}(y)
$$

The flatness provides one way to measure how far, in $L^{2}$ distance, the reduced boundary $\partial^{*} E$ of a Caccioppoli set is from the family of hyperplanes $\{y:(y-x) \cdot \nu=c\}$ inside the cylinder $\mathbf{C}(x, r, \nu)$. According to the requirements of this work, we shall prove only one property of the flatness despite it has some properties akin to the properties of the excess. 
Lemma 4.1. (Flatness and Changes of Scale) If $E \subset \mathbb{R}^{n}$ is a Caccioppoli, $x \in \mathbb{R}^{n}, r>s>0$ and $\nu \in \mathbb{S}^{n-1}$, then

$$
\boldsymbol{f}(E, x, s, \nu) \leq\left(\frac{r}{s}\right)^{n-1} \boldsymbol{f}(E, x, r, \nu)
$$

Proof. Since $\mathbf{C}(x, r, \nu) \subset \mathbf{C}(x, s, \nu)$ for all $r>s$, we have that

$$
\begin{aligned}
& \inf _{c \in \mathbb{R}} \frac{1}{s^{n-1}} \int_{\mathbf{C}(x, s, \nu) \cap \partial^{*} E} \frac{|(y-x) \cdot \nu-c|}{r^{2}} \mathrm{~d} \mathcal{H}_{n-1}(y) \leq \\
& \leq \inf _{c \in \mathbb{R}} \frac{1}{s^{n-1}} \int_{\mathbf{C}(x, r, \nu) \cap \partial^{*} E} \frac{|(y-x) \cdot \nu-c|}{r^{2}} \mathrm{~d} \mathcal{H}_{n-1}(y)
\end{aligned}
$$

Multiplying the right side by $\frac{r^{n-1}}{r^{n-1}}$, we conclude the proof.

We will not prove the reverse Poincare inequality by virtue of its very extensive proof. In the statement of the next result, the constant $\omega(n, t)$ is the constant of the Small-excess position (Theorem 2.13).

Theorem 4.2. (Reverse Poincaré Inequality) There exists a positive constant $C_{p}(n)$ such that if $E$ is a $\left(\Lambda, r_{0}\right)$ - minimizing in $C\left(x_{0}, 4 r, \nu\right)$ with $\Lambda r_{0} \leq 1, x_{0} \in \partial E, 4 r<r_{0}$ and

$$
e\left(E, x_{0}, 4 r, \nu\right) \leq \omega\left(n, \frac{1}{8}\right)
$$

then

$$
\boldsymbol{e}\left(E, x_{0}, r, \nu\right) \leq C_{p}(n)\left(\boldsymbol{f}\left(E, x_{0}, 2 r, \nu\right)+\Lambda r\right)
$$

Proof. See Theorem 24.1 in [Mag12].

\subsection{Excess revisited}

In this section, we will prove the Excess Improvement by Tilting what gives a new estimate on the excess of a almost minimizing set. In short, under the assumption that the excess of $E$ at $x_{0}$ with direction $\nu$ is bounded, the theorem provides, for each $0<\alpha<\frac{1}{200}$, a direction $\nu_{0}$ such that the excess at $x_{0}$ with direction $\nu_{0}$ in scales reduced by $\alpha$ is bounded in terms of $\alpha$ and the excess at $x_{0}$ with direction $\nu$. This result is one of the crucial steps in the proofs of the regularity theory as we will show. 
Lemma 4.3. (Excess Improvement by Tilting) Let $n \geq 3$. For all $\alpha \in\left(0, \frac{1}{200}\right)$, there exist constants $\epsilon_{2}(n, \alpha)$ and $C_{2}(n)$ such that if $E$ is $a\left(\Lambda, r_{0}\right)$ - minimizing set in $C\left(x_{0}, r, \nu\right)$ with $\Lambda r_{0} \leq 1, r<r_{0}, x_{0} \in \partial E$ and

$$
\boldsymbol{e}\left(E, x_{0}, r, \nu\right)+\Lambda r \leq \epsilon_{2}(n, \alpha)
$$

then exists $\nu_{0} \in \mathbb{S}^{n-1}$ such that

$$
\boldsymbol{e}\left(E, x_{0}, \alpha r, \nu_{0}\right) \leq C_{2}(n)\left(\alpha^{2} \boldsymbol{e}\left(E, x_{0}, r, \nu\right)+\alpha \Lambda r\right)
$$

Proof. Let us suppose that the Lemma is proved $x_{0}=0, \nu=e_{n}, r=25$. If we take $E$ a $\left(\Lambda, r_{0}\right)$ - minimizing set in $\mathbf{C}\left(x_{0}, r, \nu\right)$ with $\Lambda r_{0} \leq 1, r<$ $r_{0}, x_{0} \in \partial E$ and

$$
\mathbf{e}\left(E, x_{0}, r, \nu\right)+\Lambda r \leq \epsilon_{2}(n, \alpha)
$$

If $T$ is the linear isometry which takes $\nu$ into $e_{n}$, by 1.3 and 1.4, we know that $T\left(E_{x_{0}, r / 25}\right)$ is a $\left(\frac{\Lambda r}{25}, \frac{25 r_{0}}{r}\right)-$ minimizing set in $\mathbf{C}\left(0,25, e_{n}\right)$. From 2.6 and 2.7 , we have that

$$
\begin{gathered}
\mathbf{e}\left(T\left(E_{x_{0}, \frac{r}{25}}\right), 0,25, e_{n}\right)+\frac{\Lambda r}{25} 25=\mathbf{e}\left(E_{x_{0}, \frac{r}{25}}, 0,25, \nu\right)+\Lambda r= \\
\mathbf{e}\left(E, x_{0}, r, \nu\right)+\Lambda r \leq \epsilon_{2}(n, \alpha)
\end{gathered}
$$

Therefore, exists $\nu_{0} \in \mathbb{S}^{n-1}$ such that

$$
\mathbf{e}\left(T\left(E_{x_{0}, \frac{r}{25}}\right), 0,25 \alpha, \nu_{0}\right) \leq C_{2}(n)\left(\alpha^{2} \mathbf{e}\left(T\left(E_{x_{0}, \frac{r}{25}}\right), 0,25, e_{n}\right)+\alpha \Lambda r\right)
$$

Thus,

$$
\begin{gathered}
\mathbf{e}\left(E, x_{0}, \alpha r, T^{-1}\left(\nu_{0}\right)\right)=\mathbf{e}\left(T\left(E_{x_{0}, \frac{r}{25}}\right), 0,25 \alpha, \nu_{0}\right) \leq \\
\leq C_{2}(n)\left(\alpha^{2} \mathbf{e}\left(T\left(E_{x_{0}, \frac{r}{25}}\right), 0,25, e_{n}\right)+\alpha \Lambda r\right)= \\
=C_{2}(n)\left(\alpha^{2} \mathbf{e}\left(E, x_{0}, r, \nu\right)+\alpha \Lambda r\right)
\end{gathered}
$$

Then, the reduction that we have made allows us to prove the lemma only considering that $x_{0}=0, \nu=e_{n}, r_{0}>25$ and $E$ a $\left(\Lambda, r_{0}\right)-$ minimizing set in $\mathbf{C}\left(0,25, e_{n}\right)$ with $\Lambda r_{0} \leq 1,25<r_{0}$. Since $\frac{25}{4}<r_{0}$, provided we assume $\epsilon(n, \alpha) \leq \epsilon_{0}(n)$ and

$$
\mathbf{e}\left(E, 0,25, e_{n}\right)+25 \Lambda \leq \epsilon_{2}(n, \alpha)
$$

we are able to use the Height Bound (Theorem 2.17). Setting $M=\partial E \cap$ $\mathbf{C}\left(0,1, e_{n}\right)$, we infer that

$$
\sup \{|\mathbf{q} y|: y \in M\} \leq C_{0}(n) \mathbf{e}\left(E, 0,25, e_{n}\right)^{\frac{1}{2(n-1)}}
$$

If necessary, we reduce the size of $\epsilon_{2}(n, \alpha)$ to be less or equal than $\min \left\{\epsilon_{0}(n), \epsilon_{1}(n)\right\}$ in order to apply the Lipschtzian Approximation Theorem (Theorem 3.3 with $r=1$ ). Therefore, we find the existence of a 
Lipschitz function $u: \mathbb{R}^{n-1} \rightarrow \mathbb{R}^{n}$ with Lip $(u)<1$ such that satisfies

$$
\mathcal{H}_{n-1}(M \Delta \Gamma) \leq C_{1}(n) \mathbf{e}\left(E, 0,25, e_{n}\right)
$$

where $\Gamma=G(u) \cap \mathbf{C}\left(0,1, e_{n}\right)$. Moreover, from Proposition 3.4, we also find that

$$
\begin{gathered}
\int_{\mathbb{D}_{1}}|\nabla u|^{2} \leq C_{1}(n) \mathbf{e}\left(E, 0,25, e_{n}\right)^{\frac{1}{2(n-1)}} \\
\left|\int_{\mathbb{D}_{1}} \nabla u \cdot \nabla \phi\right| \leq C_{1}(n) \sup _{\mathbb{D}_{1}}|\nabla \phi|\left(\mathbf{e}\left(E, 0,25, e_{n}\right)+25 \Lambda\right)
\end{gathered}
$$

whenever $\phi \in C_{c}^{\infty}\left(\mathbb{D}_{1}\right)$. Setting

$$
K=C_{1}(n)\left(\mathbf{e}\left(E, 0,25, e_{n}\right)+25 \Lambda\right) \text { and } u_{0}=\frac{u}{\sqrt{K}}
$$

we obtain a Lipschitz function $u_{0}$ which, by 4.7 and 4.8 , satisfies both

$$
\left\|u_{0}\right\|_{L^{2}\left(\mathbb{D}_{1}\right)}^{2}=\int_{\mathbb{D}_{1}}\left|\nabla u_{0}\right|^{2} \leq \frac{C_{1}(n) \mathbf{e}\left(E, 0,25, e_{n}\right)}{K} \leq 1
$$

and

$$
\begin{gathered}
\left|\int_{\mathbb{D}_{1}} \nabla u_{0} \cdot \nabla \phi\right| \leq \sup _{\mathbb{D}_{1}}|\nabla \phi| \frac{C_{1}(n)\left(\mathbf{e}\left(E, 0,25, e_{n}\right)+25 \Lambda\right)}{\sqrt{K}} \leq \\
\leq \sqrt{K} \sup _{\mathbb{D}_{1}}|\nabla \phi|
\end{gathered}
$$

Then $u_{0} \in W^{1,2}\left(\mathbb{D}_{1}\right)$ and we can approximate $u_{0}$, in $L^{2}\left(\mathbb{D}_{1}\right)$-norm, by a harmonic function $v$, i.e. $u_{0}$ is in the conditions of Lemma 3.6 (setting $\tau=\alpha^{n+3}$ ) provided we assume

$$
\sqrt{K} \leq 4.4 \sqrt{C_{1}(n) \epsilon_{2}(n, \alpha)} \leq \sigma\left(\alpha^{n+3}\right)
$$

Thus there exists $v$ harmonic function on $\mathbb{D}_{1}$ such that $\|v\|_{L^{2}\left(\mathbb{D}_{1}\right)} \leq 1$ and

$$
\int_{\mathbb{D}_{1}}\left|v-u_{0}\right|^{2} \leq \alpha^{n+3}
$$

If we set $v_{0}=\sqrt{K} v$, we get a harmonic function $v_{0}$ on $\mathbb{D}_{1}$ with $\left\|v_{0}\right\|_{L^{2}\left(\mathbb{D}_{1}\right)} \leq$ $\sqrt{K}$ and

$$
\int_{\mathbb{D}_{1}}\left|v_{0}-u\right|^{2} \leq K \alpha^{n+3}
$$

Since $100 \alpha<\frac{1}{2}$, from Lemma 3.5, we have that

$$
\begin{aligned}
\sup _{\mathbb{D}_{100 \alpha}}\left|v_{0}-w\right| & \leq \frac{(100 \alpha)^{2}}{\omega_{n}} \overbrace{\left\|\nabla v_{0}\right\|_{L^{2}\left(\mathbb{D}_{1}\right)}}^{\leq \sqrt{K}} \leq \\
& \leq \sqrt{K} \frac{(100 \alpha)^{2}}{\omega_{n}}
\end{aligned}
$$


where $w(z)=v_{0}(0)+\nabla v_{0}(0) \cdot z, z \in \mathbb{D}_{1}$. By the last inequality, 4.9 and $(a+b)^{2} \leq 2 a^{2}+2 b^{2}, \forall a, b>0$, we conclude that

$$
\begin{gathered}
\int_{\mathbb{D}_{100 \alpha}}|u-w|^{2}=\|u-w\|_{L^{2}\left(\mathbb{D}_{100 \alpha}\right)}^{2} \leq\left(\left\|u-v_{0}\right\|_{L^{2}\left(\mathbb{D}_{100 \alpha}\right)}+\left\|v_{0}-w\right\|_{L^{2}\left(\mathbb{D}_{100 \alpha}\right)}\right)^{2} \leq \\
\leq 2\left\|u-v_{0}\right\|_{L^{2}\left(\mathbb{D}_{100 \alpha}\right)}^{2}+2\left\|v_{0}-w\right\|_{L^{2}\left(\mathbb{D}_{100 \alpha}\right)}^{2} \leq \\
\leq 2 K \alpha^{n+3}+2 \mathcal{H}_{n-1}\left(\mathbb{D}_{100 \alpha}\right)\left(\sqrt{K} \frac{(100 \alpha)^{2}}{\omega_{n}}\right)^{2}= \\
=2 K\left(\alpha^{n+3}+\frac{2 \omega_{n-1} 100^{n+3} \alpha^{n+3}}{\omega_{n}^{2}}\right)
\end{gathered}
$$

Therefore

$$
\frac{1}{\alpha^{n+1}} \int_{\mathbb{D}_{100 \alpha}}|u-w|^{2} \leq A(n) K \alpha^{2}
$$

where we set $A(n)=2\left(1+\frac{2 \omega_{n-1} 100^{n+3}}{\omega_{n}^{2}}\right)$. We aim to prove that

$$
\nu_{0}=\frac{\left(-\nabla v_{0}(0), 1\right)}{\sqrt{1+\left|\nabla v_{0}(0)\right|^{2}}}
$$

is the direction we have searched for. For this purpose, let us state two claims which will be proved later.

Claim 1: If $K^{\frac{1}{n-1}} \leq \alpha^{n+3}$, we have that

$$
\mathbf{f}\left(E, 0,100 \alpha, \nu_{0}\right) \leq A_{1}(n) K \alpha^{2}
$$

Claim 2: If we take $K^{\frac{1}{n-1}} \leq \alpha^{n+3}$ and $\epsilon_{2}(n, \alpha)$ suitably small, we find that

$$
\mathbf{e}\left(E, 0,100 \alpha, \nu_{0}\right) \leq \omega\left(n, \frac{1}{8}\right)
$$

We assume that $K$ and $\epsilon_{2}(n, \alpha)$ are satisfying the requests in the Claims. Since $100 \alpha<r_{0}$ and $\mathbf{C}\left(0,100 \alpha, \nu_{0}\right) \subset \mathbf{C}\left(0,25, e_{n}\right), E$ is a $\left(\Lambda, r_{0}\right)-$ minimizing set in $\mathbf{C}\left(0,100 \alpha, \nu_{0}\right)$. Then, from 4.12 , we are in the conditions to apply the reverse Poincaré inequality (Theorem 4.2) and deduce that

$$
\mathbf{e}\left(E, 0,25 \alpha, \nu_{0}\right) \leq C_{p}(n)\left(\mathbf{f}\left(E, 0,50 \alpha, \nu_{0}\right)+25 \Lambda \alpha\right)
$$

By 4.11, the change of scale on the flatness (Proposition 4.1) and the last inequality

$$
\begin{gathered}
\mathbf{e}\left(E, 0,25 \alpha, \nu_{0}\right) \leq \\
\leq C_{p}(n)\left(\mathbf{f}\left(E, 0,50 \alpha, \nu_{0}\right)+25 \Lambda \alpha\right) \leq \\
\leq C_{p}(n)\left(2^{n-1} \mathbf{f}\left(E, 0,100 \alpha, \nu_{0}\right)+25 \Lambda \alpha\right) \leq C_{p}(n)\left(2^{n-1} A_{1}(n) K \alpha^{2}+25 \Lambda \alpha\right)
\end{gathered}
$$


Recalling the definition of $K$ and $\alpha<\frac{1}{200}<25$, we get that

$$
\mathbf{e}\left(E, 0,25 \alpha, \nu_{0}\right) \leq
$$

$$
\begin{gathered}
\leq \overbrace{\max \left\{C_{p}(n) 2^{n-1} A_{1}(n) C_{1}(n), C_{p}(n)\right\}}^{\doteq C^{2}(n)}\left(\alpha^{2} \mathbf{e}\left(E, 0,25, e_{n}\right)+\Lambda \alpha^{2}+25 \Lambda \alpha\right) \leq \\
\leq 2 C^{2}(n)\left(\alpha^{2} \mathbf{e}\left(E, 0,25, e_{n}\right)+25 \Lambda \alpha\right)
\end{gathered}
$$

what gives the wished direction and conclude the proof of the lemma. Let us prove the claims.

Proof of Claim 1: Suppose that $K^{\frac{1}{n-1}} \leq \alpha^{n+3}$ and set

$$
c_{0}=\frac{v_{0}(0)}{\sqrt{1+\left|\nabla v_{0}(0)\right|^{2}}}
$$

By the $\mathcal{H}_{n-1}$-equivalence of $\partial E$ and $\partial^{*} E$ in $\mathbf{C}\left(0,25, e_{n}\right)$ and the definition of flatness, we deduce that

$$
\mathbf{f}\left(E, 0,100 \alpha, \nu_{0}\right) \leq \frac{1}{(100 \alpha)^{n+1}} \int_{M \cap \mathbf{C}\left(0,100 \alpha, \nu_{0}\right)}\left|y \cdot \nu_{0}-c_{0}\right|^{2} \mathcal{H}_{n-1}(y)
$$

We want to estimate the right side of the inequality in order to prove the claim. For this purpose, we will estimate the integral in both inside and outside the graph of u, i.e. in $M \cap \Gamma \cap \mathbf{C}\left(0,100 \alpha, \nu_{0}\right)$ and $(M \backslash \Gamma) \cap$ $\mathbf{C}\left(0,100 \alpha, \nu_{0}\right)$.

Step 1: If $y=(z, u(z)) \in \Gamma$, putting the definitions of $\nu_{0}, c_{0}$ and $w$ into account we find that

$$
\begin{gathered}
\left|y \cdot \nu_{0}-c_{0}\right|^{2}=\frac{\left|-z \cdot \nabla v_{0}(0)+u(z)-v_{0}(0)\right|^{2}}{1+\left|\nabla v_{0}(0)\right|^{2}}= \\
=\frac{|u(z)-w(z)|^{2}}{1+\left|\nabla v_{0}(0)\right|^{2}} \leq|u(z)-w(z)|^{2}
\end{gathered}
$$

Therefore

$$
\begin{gathered}
\frac{1}{(100 \alpha)^{n+1}} \int_{M \cap \Gamma \cap \mathbf{C}\left(0,100 \alpha, \nu_{0}\right)}\left|y \cdot \nu_{0}-c_{0}\right|^{2} \mathcal{H}_{n-1}(y) \leq \\
\frac{1}{(100 \alpha)^{n+1}} \int_{M \cap \Gamma \cap \mathbf{C}\left(0,100 \alpha, \nu_{0}\right)}|u(z)-w(z)|^{2} \mathcal{H}_{n-1}(y) \leq \\
\leq{ }^{4.10} A(n) K \alpha^{2}
\end{gathered}
$$

Step 2: We shall provided an estimate for $(M \backslash \Gamma) \cap \mathbf{C}\left(0,100 \alpha, \nu_{0}\right)$. Keeping in mind the definitions of $\nu_{0}$ and $c_{0}$, we find that

$$
\frac{1}{(100 \alpha)^{n+1}} \int_{M \backslash \Gamma \cap \mathbf{C}\left(0,100 \alpha, \nu_{0}\right)}\left|y \cdot \nu_{0}-c_{0}\right|^{2} \mathcal{H}_{n-1}(y)=
$$




$$
\begin{aligned}
& =\frac{1}{(100 \alpha)^{n+1}} \int_{M \backslash \Gamma \cap \mathbf{C}\left(0,100 \alpha, \nu_{0}\right)} \frac{\left|-\mathbf{p} y \cdot \nabla v_{0}(0)+\mathbf{q} y+v_{0}(0)\right|^{2}}{1+\left|\nabla v_{0}(0)\right|^{2}} \mathcal{H}_{n-1}(y) \leq \\
& \leq^{*} \frac{1}{(100 \alpha)^{n+1}} \int_{M \backslash \Gamma \cap \mathbf{C}\left(0,100 \alpha, \nu_{0}\right)}\left|\mathbf{p} y \cdot \nabla v_{0}(0)\right|^{2}+|\mathbf{q} y|^{2}+\left|v_{0}(0)\right|^{2} \mathcal{H}_{n-1}(y) \leq \\
& \leq \frac{1}{(100 \alpha)^{n+1}} \mathcal{H}_{n-1}(M \backslash \Gamma)\left(\sup _{y \in M}\left(|\mathbf{q} y|^{2}\right)+\sup _{y \in M}\left(\left|\mathbf{p} y \cdot \nabla v_{0}(0)\right|^{2}\right)+\left|v_{0}(0)\right|^{2}\right)
\end{aligned}
$$

where in $\left(^{*}\right)$ we have used that $\frac{1}{1+\left|\nabla v_{0}(0)\right|^{2}} \leq 1$, the triangle inequality and $(a+b+c)^{2} \leq 2 a^{2}+2 b^{2}+2 c^{2}, \forall a, b, c>0$. By 4.5, 4.6 and the definition of $K$, we have that

$$
\begin{array}{r}
\frac{1}{(100 \alpha)^{n+1}} \int_{M \backslash \Gamma \cap \mathbf{C}\left(0,100 \alpha, \nu_{0}\right)}\left|y \cdot \nu_{0}-c_{0}\right|^{2} \mathcal{H}_{n-1}(y) \leq \\
\frac{C_{1}(n) \mathbf{e}\left(E, 0,25, e_{n}\right)}{(100 \alpha)^{n+1}}\left(C_{0}(n)^{2} \mathbf{e}\left(E, 0,25, e_{n}\right)^{\frac{1}{(n-1)}}+\right. \\
\left.+\sup _{y \in M}\left(\left|\mathbf{p} y \cdot \nabla v_{0}(0)\right|^{2}\right)+\left|v_{0}(0)\right|^{2}\right) \leq \\
\leq \frac{A^{1}(n) K}{\alpha^{n+1}}\left(K^{\frac{1}{n-1}}+\sup _{y \in M}\left(\left|\mathbf{p} y \cdot \nabla v_{0}(0)\right|^{2}\right)+\left|v_{0}(0)\right|^{2}\right)
\end{array}
$$

where we have set $A^{1}(n)=\frac{C_{1}(n)}{100^{n+1}} \max \left\{C_{0}(n)^{2}, 1\right\}$. In order to finish this step, we shall estimate $\sup _{y \in M}\left(\left|\mathbf{p} y \cdot \nabla v_{0}(0)\right|^{2}\right)+\left|v_{0}(0)\right|^{2}$. Note that $\mid \mathbf{p} y$. $\left.\nabla v_{0}(0)\right|^{2} \leq|\mathbf{p} y|^{2}\left|\nabla v_{0}(0)\right|^{2} \leq\left|\nabla v_{0}(0)\right|^{2}$, then we can reduce our effort to estimate $\left|\nabla v_{0}(0)\right|^{2}+\left|v_{0}(0)\right|^{2}$. From the mean value property of harmonic functions (3.29), we obtain

$$
\begin{gathered}
\left|v_{0}(0)\right|^{2}=\left|\underset{\mathbb{D}_{1}}{f} v_{0}\right|^{2} \leq\left(\underset{\mathbb{D}_{1}}{\left.f\left|v_{0}\right|\right)^{2} \leq}\right. \\
\leq^{*} \frac{1}{\omega_{n-1}^{2}}\left\|v_{0}\right\|_{L^{2}\left(\mathbb{D}_{1}\right)}^{2}\left(\int_{\mathbb{D}_{1}} 1\right)=\frac{1}{\omega_{n-1}}\left\|v_{0}\right\|_{L^{2}\left(\mathbb{D}_{1}\right)}^{2}
\end{gathered}
$$

where in $\left(^{*}\right)$ we have used Holder's inequality (6.2 in [Fol99, p. 174]). From 3.30, we get

$$
\left|\nabla v_{0}(0)\right|^{2} \leq H(n)\left\|v_{0}\right\|_{L^{2}\left(\mathbb{D}_{1}\right)}^{2}
$$

The last two inequalities ensure that

$$
\begin{gathered}
\left|v_{0}(0)\right|^{2}+\left|\nabla v_{0}(0)\right|^{2} \leq \overbrace{2 \max \left\{\frac{1}{\omega_{n}}, \frac{1}{\omega_{n-1}}\right\}}^{\doteq A^{\prime}(n)}\left\|v_{0}\right\|_{L^{2}\left(\mathbb{D}_{1}\right)}^{2} \leq \\
\leq A^{\prime}(n)\left(\left\|u-v_{0}\right\|_{L^{2}\left(\mathbb{D}_{1}\right)}^{2}+\|u\|_{L^{2}\left(\mathbb{D}_{1}\right)}^{2}\right) \leq \\
\leq_{4.7}^{4.9} A^{\prime}(n)\left(K \alpha^{n+3}+C_{1}(n) \mathbf{e}\left(E, 0,25, e_{n}\right)^{\frac{1}{n-1}}\right) \leq A^{\prime \prime}(n)\left(K \alpha^{n+3}+K^{\frac{1}{n-1}}\right)
\end{gathered}
$$

Finally, by the last inequality, 4.13 and $K^{\frac{1}{n-1}} \leq \alpha^{n+3}$, we conclude the 
proof of this step as follows

$$
\begin{gathered}
\frac{1}{(100 \alpha)^{n+1}} \int_{M \backslash \Gamma \cap \mathbf{C}\left(0,100 \alpha, \nu_{0}\right)}\left|y \cdot \nu_{0}-c_{0}\right|^{2} \mathcal{H}_{n-1}(y) \leq \\
\leq \frac{A^{1}(n) K}{\alpha^{n+1}}\left(\left(1+A^{\prime \prime}(n)\right) K^{\frac{1}{n-1}}+A^{\prime \prime}(n) K \alpha^{n+3}\right) \leq \\
\leq \overbrace{A^{1}(n)\left(1+A^{\prime \prime}(n)\right)}^{\doteq A_{1}(n)} K \alpha^{2}
\end{gathered}
$$

Proof of Claim 2: Note that $100 \sqrt{2} \alpha<r_{0}$ and $\mathbf{B}(0,200 \alpha) \Subset$ $\mathbf{C}\left(0,25, e_{n}\right)$, then, from Proposition 2.9, we find that

$$
\mathbf{e}\left(E, 0,100 \alpha, \nu_{0}\right) \leq C_{d}(n)\left(\mathbf{e}\left(E, 0,100 \sqrt{2} \alpha, e_{n}\right)+\left|\nu_{0}-e_{n}\right|^{2}\right)
$$

By the definition of $\nu_{0}$, we find that

$$
\begin{gathered}
\left|\nu_{0}-e_{n}\right|^{2}=\frac{\left|\nabla v_{0}(0)\right|^{2}+\left(1-\sqrt{1+\left|\nabla v_{0}(0)\right|^{2}}\right)^{2}}{1+\left|\nabla v_{0}(0)\right|^{2}} \leq \\
\leq^{*}\left|\nabla v_{0}(0)\right|^{2}+\frac{\left|\nabla v_{0}(0)\right|^{4}}{4} \leq^{* *} \frac{5}{4}\left|\nabla v_{0}(0)\right|^{2} \leq^{* * *} C^{\prime}(n) K
\end{gathered}
$$

where in $(*)$ we have used that $\frac{1}{1+\left|\nabla v_{0}(0)\right|^{2}} \leq 1$ and $\sqrt{1+s}-1 \leq \frac{s}{2}, \forall s>0$ with $\left|\nabla v_{0}(0)\right|^{2}$ in place of $s$, in $(* *)$ and $(* * *)$ we took $\left\|v_{0}\right\|_{L^{2}\left(\mathbb{D}_{1}\right)}^{2} \leq K \leq$ $\alpha^{(n-3)(n-1)}<1$ into account. Using the change of scale of the excess (Proposition 2.5) we produce $\mathbf{e}\left(E, 0,100 \sqrt{2} \alpha, e_{n}\right) \leq\left(\frac{25}{100 \sqrt{2} \alpha}\right)^{2} \mathbf{e}\left(E, 0,25, e_{n}\right)$. Putting it all together and recalling that $\mathbf{e}\left(E, 0,25, e_{n}\right) \leq \frac{K}{C_{1}(n)}$ (from the definition of $K$ ), we get that

$$
\begin{gathered}
\mathbf{e}\left(E, 0,100 \alpha, \nu_{0}\right) \leq C_{d}(n)\left(\left(\frac{25}{100 \sqrt{2} \alpha}\right)^{2} \mathbf{e}\left(E, 0,25, e_{n}\right)+C^{\prime}(n) K\right) \leq \\
\leq C_{d}(n)\left(\left(\frac{25}{100 \sqrt{2} \alpha}\right)^{2} \frac{K}{C_{1}(n)}+C^{\prime}(n) K\right)=K C(n, \alpha)
\end{gathered}
$$

what ensures the proof of the Claim 2 provided we take $\epsilon_{2}(n, \alpha)$ small enough in order to have $K C(n, \alpha) \leq \omega\left(n, \frac{1}{8}\right)$.

We aim to refine the Excess Improvement by Tilting providing a result such that ensures the existence of a direction $\nu_{0}$ with certain properties as exhibited before and, furthermore, also enables to control the distance of $\nu_{0}$ from the direction $\nu$ which has the bounded excess (that is 4.15 . To achieve this goal, we must replace the exponent 2 in 4.3 with a smaller one $2 \gamma\left(0<\gamma<\frac{1}{2}\right)$. For that reason, the constant $\alpha^{2 \gamma}$ will be bigger than $\alpha^{2}$, therefore, it makes the estimate 4.3 weaker than 4.14 .

Theorem 4.4. (Excess Improvement) Let $n \geq 3$. For every $\gamma \in$ $\left(0, \frac{1}{2}\right)$, there exist positive constants $\alpha_{0}(n, \gamma)<1, \epsilon_{3}(n, \gamma)$ and $C_{3}(n, \gamma)$ such that if $E$ is a $\left(\Lambda, r_{0}\right)$ - minimizing set in $C\left(x_{0}, r, \nu\right)$ with $\Lambda r_{0} \leq$ 
$1, r<r_{0}, x_{0} \in \partial E$ and

$$
\mathcal{E}_{x_{0}, \nu}(r) \leq \epsilon_{3}(n, \gamma)
$$

where we have set $\mathcal{E}_{x, \eta}(s) \doteq \max \left\{\boldsymbol{e}(E, x, s, \nu), \frac{\Lambda s}{\alpha_{0}^{n-1+2 \gamma}}\right\}$ for all $x \in \mathbb{R}^{n}, s>$ 0 and $\eta \in \mathbb{S}^{n-1}$, then there exists $\nu_{0} \in \mathbb{S}^{n-1}$ such that

$$
\begin{gathered}
\mathcal{E}_{x_{0}, \nu_{0}}\left(\alpha_{1}(n, \gamma) r\right) \leq \alpha_{0}(n, \gamma)^{2 \gamma} \mathcal{E}_{x_{0}, \nu}(r) \\
\left|\nu_{0}-\nu\right|^{2} \leq C_{3}(n, \gamma) \mathcal{E}_{x_{0}, \nu}(r)
\end{gathered}
$$

Proof. Let $C_{2}(n)$ be the constant provided by the Excess Improvement by Tilting (Lemma 4.3), we define $\alpha_{0}(n, \gamma) \doteq \min \left\{\frac{1}{200},\left(\frac{1}{2 C_{2}(n)}\right)^{\frac{1}{1-2 \gamma}}\right\}$. For the sake of brevity denote $\alpha_{0}=\alpha_{0}(n, \gamma)$, then Lemma 4.3 also provides a constant $\epsilon_{2}\left(n, \alpha_{0}\right)$ which we use to define $\epsilon_{3}(n, \gamma)=\frac{\epsilon_{2}\left(n, \alpha_{0}\right)}{2}$. Taking $E$ in the conditions above, since $2 \gamma<1$, we obtain that

$$
\alpha_{0} \frac{\Lambda r}{\alpha_{0}^{n-1+2 \gamma}} \leq \alpha_{0} \mathcal{E}_{x_{0}, \nu}(r) \leq \alpha_{0}^{2 \gamma} \mathcal{E}_{x_{0}, \nu}(r)
$$

In order to prove 4.14, it remains to exhibit $\nu_{0}$ such that $\mathbf{e}\left(E, x_{0}, \alpha_{0} r, \nu_{0}\right) \leq$ $\alpha_{0}^{2 \gamma} \mathcal{E}_{x_{0}, \nu}(r)$. If $\mathbf{e}\left(E, x_{0}, r, \nu\right) \leq \Lambda r, \nu$, which clearly satisfies 4.15 , is the desired direction. Indeed, by the Excess and Changes of Scale (Proposition 2.5 ), we find that

$$
\begin{gathered}
\mathbf{e}\left(E, x_{0}, \alpha_{0} r, \nu\right) \leq \frac{1}{\alpha_{0}^{n-1}} \mathbf{e}\left(E, x_{0}, r, \nu\right) \leq \frac{\alpha_{0}^{2 \gamma}}{\alpha_{0}^{n-1+2 \gamma}} \Lambda r \leq \\
\leq \alpha_{0}^{2 \gamma} \mathcal{E}_{x_{0}, \nu}(r)
\end{gathered}
$$

Now, we suppose that $\Lambda r \leq \mathbf{e}\left(E, x_{0}, r, \nu\right)$, then

$$
\begin{gathered}
\mathbf{e}\left(E, x_{0}, r, \nu\right)+\Lambda r \leq 2 \mathbf{e}\left(E, x_{0}, r, \nu\right) \leq 2 \mathcal{E}_{x_{0}, \nu}(r) \leq \\
\leq 2 \epsilon_{3}(n, \gamma) \leq{ }^{*} \epsilon_{2}\left(n, \alpha_{0}\right)
\end{gathered}
$$

where $(*)$ follows from our choice of $\epsilon_{3}(n, \gamma)$. Thus, we are in position to apply the Excess Improvement by Tilting. Since $2 C_{2}(n) \leq \frac{1}{\alpha_{0}^{1-2 \gamma}}$, by our assumption, we can conclude the proof of 4.14 as follows

$$
\begin{array}{r}
\mathbf{e}\left(E, x_{0}, \alpha_{0} r, \nu_{0}\right) \leq{ }^{4.3} C_{2}(n)\left(\alpha_{0}^{2} \mathbf{e}\left(E, x_{0}, r, \nu\right)+\alpha_{0} \Lambda r\right) \leq \\
\leq{ }^{\alpha_{0}<1} 2 C_{2}(n) \alpha_{0} \mathbf{e}\left(E, x_{0}, r, \nu\right) \leq 2 C_{2}(n) \alpha_{0} \mathcal{E}_{x_{0}, \nu}(r) \leq \alpha_{0}^{2 \gamma} \mathcal{E}_{x_{0}, \nu}(r)
\end{array}
$$

Let us prove that 4.15 holds true for the direction $\nu_{0}$ provided by the Excess Improvement by Tilting. Taking into account that $(a+b)^{2} \leq$ $2\left(a^{2}+b^{2}\right)$, the triangle inequality furnishes

$$
\left|\nu-\nu_{0}\right|^{2} \leq 2\left(\left|\nu-\nu_{E}\right|^{2}+\left|\nu_{0}-\nu_{E}\right|^{2}\right)
$$


Thus, by definition of the excess,

$$
\begin{array}{r}
\left|\nu-\nu_{0}\right|^{2} \mathcal{H}_{n-1}\left(\partial^{*} E \cap \mathbf{C}\left(x_{0}, \alpha_{0} r, \nu_{0}\right)\right) \leq \\
\leq 2 \int_{\partial^{*} E \cap \mathbf{C}\left(x_{0}, \alpha_{0} r, \nu_{0}\right)}\left(\left|\nu_{0}-\nu_{E}\right|^{2}+\left|\nu-\nu_{E}\right|^{2}\right) \mathrm{d} \mathcal{H}_{n-1}= \\
=2\left(\alpha_{0} r\right)^{n-1} \mathbf{e}\left(E, x_{0}, \alpha_{0} r, \nu_{0}\right)+2 \int_{\partial^{*} E \cap \mathbf{C}\left(x_{0}, \alpha_{0} r, \nu_{0}\right)}\left|\nu-\nu_{E}\right|^{2} \mathrm{~d} \mathcal{H}_{n-1}
\end{array}
$$

We note that $\sqrt{2} \alpha_{0} r \leq \frac{\sqrt{2}}{200} r<r<r_{0}$ implies

$$
\mathbf{C}\left(x_{0}, \alpha_{0} r, \nu_{0}\right) \subset \mathbf{B}\left(x_{0}, \sqrt{2} \alpha_{0} r\right) \subset \mathbf{B}\left(x_{0}, r\right) \subset \mathbf{C}\left(x_{0}, r, \nu\right)
$$

The first inclusion in 4.18 makes possible to apply the Density Estimates for Cylinders (Corollary 1.8). Since

$$
\mathcal{H}_{n-1}\left(\partial^{*} E \cap \mathbf{C}\left(x_{0}, \alpha_{0} r, \nu_{0}\right)\right)=\mathcal{P}\left(E, \mathbf{C}\left(x_{0}, \alpha_{0} r, \nu_{0}\right)\right)
$$

the density estimate for cylinders ensures that

$$
c(n)\left(\alpha_{0} r\right)^{n-1} \leq \mathcal{P}\left(E, \mathbf{C}\left(x_{0}, \alpha_{0} r, \nu\right)\right)
$$

By 4.18 and the last inequality, the inequality 4.17 became

$$
\begin{gathered}
c(n)\left(\alpha_{0} r\right)^{n-1}\left|\nu-\nu_{0}\right|^{2} \leq \\
\leq 2\left(\alpha_{0} r\right)^{n-1} \mathbf{e}\left(E, x_{0}, \alpha_{0} r, \nu_{0}\right)+2 \int_{\partial^{*} E \cap \mathbf{C}\left(x_{0}, r, \nu\right)}\left|\nu-\nu_{E}\right|^{2} \mathrm{~d} \mathcal{H}_{n-1}= \\
=2\left(\alpha_{0} r\right)^{n-1} \mathbf{e}\left(E, x_{0}, \alpha_{0} r, \nu_{0}\right)+2 r^{n-1} \mathbf{e}\left(E, x_{0}, r, \nu\right)
\end{gathered}
$$

By the definition of $\mathcal{E}_{x_{0}, \nu}(r)$ and 4.16 , we find that

$$
\begin{gathered}
c(n)\left(\alpha_{0} r\right)^{n-1}\left|\nu-\nu_{0}\right|^{2} \leq 2\left(\alpha_{0} r\right)^{n-1} \alpha_{0}^{2 \gamma} \mathcal{E}_{x_{0}, \nu}(r)+2 r^{n-1} \mathbf{e}\left(E, x_{0}, r, \nu\right) \leq \\
\leq 2 r^{n-1}\left(\alpha_{0}^{n-1} \alpha_{0}^{2 \gamma}+1\right) \mathcal{E}_{x_{0}, \nu}(r)
\end{gathered}
$$

Set $C_{3}(n, \gamma) \doteq \frac{2\left(\alpha_{0}^{2 \gamma}+\alpha_{0}^{1-n}\right)}{c(n)}$ to conclude the proof of 4.15 . 


\section{3 $C^{1, \gamma}$-regularity of the almost minimizing sets}

We finally have evolved all the needed tools to reach the main goal of this work. For any $\gamma \in(0,1 / 2)$, we take $E$ an almost minimizing set and $x_{0} \in \partial E$, if we suppose that $E$ content a boundedness condition (depending on $\gamma$ and the dimension $n$ ) on the excess at $x_{0}$ at scale $25 r$, we can exhibit a function $u \in C^{1, \gamma}$ whose graph coincides with $\partial E$ in $\mathbf{C}\left(x_{0}, r, e_{n}\right)$. Moreover, the Holder constant of $u$ will depend on the excess at $x_{0}$ at scale $25 r$.

Theorem 4.5. ( $C^{1, \gamma}$-regularity of the almost minimizing sets) Let $n \geq 3$. For every $\gamma \in\left(0, \frac{1}{2}\right)$, there exist $\epsilon_{4}(n, \gamma)$ and $C_{4}(n, \gamma)$ such that if $E$ is a $\left(\Lambda, r_{0}\right)$ - minimizing set in $C\left(x_{0}, 25 r, e_{n}\right)$ with $\Lambda r_{0} \leq 1, x_{0} \in$ $\partial E, 25 r<r_{0}$ and

$$
\boldsymbol{e}\left(E, x_{0}, 25 r, e_{n}\right)+\Lambda r \leq \epsilon_{4}(n, \gamma)
$$

then there exists a Lipschitz function $u: \mathbb{R}^{n} \rightarrow \mathbb{R}$ with Lip $(u)<1$ such that $u \in C^{1, \gamma}\left(\boldsymbol{p} x_{0}+\mathbb{D}_{r}\right)$,

$$
\begin{gathered}
\sup \frac{|u|}{r} \leq C_{1}(n) \boldsymbol{e}\left(E, x_{0}, 25 r, e_{n}\right)^{\frac{1}{2(n-1)}} \\
\boldsymbol{C}\left(x_{0}, r, e_{n}\right) \cap \partial E=\left(x_{0}+G(u)\right) \cap \boldsymbol{C}\left(x_{0}, r, e_{n}\right)
\end{gathered}
$$

and either

$$
C\left(x_{0}, r, e_{n}\right) \cap(E \backslash \partial E)=C\left(x_{0}, r, e_{n}\right) \cap\left(x_{0}+\text { hypo }(u)\right)
$$

or

$$
\boldsymbol{C}\left(x_{0}, r, e_{n}\right) \cap(E \backslash \partial E)=\boldsymbol{C}\left(x_{0}, r, e_{n}\right) \cap\left(x_{0}+\operatorname{epi}(u)\right)
$$

Furthermore, $\nu_{E}$ is a Holder function in $C\left(x_{0}, r, e_{n}\right) \cap \partial E$ and both $\nu_{E}$ and $\nabla u$ have Holder constant equal to $\frac{C_{4}(n, \gamma)}{r^{\gamma}} \sqrt{\boldsymbol{e}\left(E, x_{0}, 25 r, e_{n}\right)+\Lambda r}$.

Proof. We should use the same notation presented in the Excess Improvement Theorem (Theorem 4.4), precisely $\mathcal{E}_{x, \eta}(s) \doteq \max \left\{\mathbf{e}(E, x, s, \nu), \frac{\Lambda s}{\alpha_{0}^{n-1+2 \gamma}}\right\}$ for all $x \in \mathbb{R}^{n}, s>0$ and $\eta \in \mathbb{S}^{n-1}$.

Claim 1: For any $x \in \mathbf{C}\left(x_{0}, r, e_{n}\right) \cap \partial E$, there exists $\nu(x) \in \mathbb{S}^{n-1}$ such that

$$
\begin{gathered}
\mathcal{E}_{x, \nu(x)}(s) \leq C_{3}^{\prime}(n, \gamma)\left(\frac{s}{r}\right)^{2 \gamma} \mathcal{E}_{x_{0}, e_{n}}(25 r) \quad \forall s \in(0,12 r) \\
\left|\nu(x)-e_{n}\right|^{2} \leq C_{3}^{\prime}(n, \gamma) \mathcal{E}_{x_{0}, e_{n}}(25 r)
\end{gathered}
$$




$$
\mathcal{E}_{x, e_{n}}(s) \leq C_{3}^{\prime}(n, \gamma) \mathcal{E}_{x_{0}, e_{n}}(25 r) \quad \forall s \in(0,24 r)
$$

Recalling the Lipschitz Approximation Theorem (Theorem 3.3), we have

$$
M_{0}=\left\{x \in \mathbf{C}\left(x_{0}, r, e_{n}\right) \cap \partial E: \sup _{0<s<8 r} \mathbf{e}\left(E, x, s, e_{n}\right) \leq \delta_{0}(n)\right\}
$$

We require $\epsilon_{4}(n, \gamma)$ to be smaller than both $\epsilon_{1}(n)$ and $\delta_{0}(n)$, thus exists a Lipschitz functions $u: \mathbb{R}^{n} \rightarrow \mathbb{R}$ with $\operatorname{Lip}(u)<1$ such that 4.20 holds true and, by 4.26 ,

$$
M_{0}=\mathbf{C}\left(x_{0}, r, e_{n}\right) \cap \partial E \subset G(u) \cap \mathbf{C}\left(x_{0}, r, e_{n}\right)
$$

what put us in position to apply the Lipschitz Boundary Criterion (Theorem 3.1), then

$$
\mathbf{C}\left(x_{0}, r, e_{n}\right) \cap \partial E=G(u) \cap \mathbf{C}\left(x_{0}, r, e_{n}\right)
$$

and either

$$
\mathbf{C}\left(x_{0}, r, e_{n}\right) \cap(E \backslash \partial E)=\mathbf{C}\left(x_{0}, r, e_{n}\right) \cap\left(x_{0}+\text { hypo }(u)\right)
$$

or

$$
\mathbf{C}\left(x_{0}, r, e_{n}\right) \cap(E \backslash \partial E)=\mathbf{C}\left(x_{0}, r, e_{n}\right) \cap\left(x_{0}+\operatorname{epi}(u)\right)
$$

holds true, that is either 4.22 or 4.23 . In order to prove the Holder continuity of $\nabla u$ and $\nu_{E}$, we set

$$
(\nabla u)_{z, s}=\frac{1}{\left|z+\mathbb{D}_{s}\right|} \int_{z+\mathbb{D}_{s}} \nabla u
$$

and we establish the last claim of this proof.

Claim 2: For any $s \in(0, r)$ and $z \in\left(\mathbf{p} x_{0}+\mathbb{D}_{r}\right)$, it holds that

$$
\frac{1}{s^{n-1}} \int_{z+\mathbb{D}_{s}}\left|\nabla u-(\nabla u)_{z, s}\right|^{2} \leq K(n, \gamma)\left(\frac{s}{r}\right)^{2 \gamma} \mathcal{E}_{x_{0}, e_{n}}(25 r)
$$

The Claim 2 put us in position to apply the Campanato's Criterion (Theorem 7.51 in [LA00]) and thus exists $K^{\prime}(n, \gamma)$ such that

$$
\left|u(z)-u\left(z^{\prime}\right)\right| \leq K^{\prime}(n \gamma) \sqrt{\mathcal{E}_{x_{0}, e_{n}}(25 r)}\left(\frac{\left|z-z^{\prime}\right|}{r}\right)^{\gamma} \forall z, z^{\prime} \in\left(\mathbf{p} x_{0}+\mathbb{D}_{r}\right)
$$

By the very definition of $\mathcal{E}$, we set $C_{4}^{\prime}(n, \gamma)=K^{\prime}(n, \gamma) \max \left\{1, \frac{25}{\alpha_{0}^{n-1+2 \gamma}}\right\}$ and then, $\forall z, z^{\prime} \in\left(\mathbf{p} x_{0}+\mathbb{D}_{r}\right)$, we have

$$
\left|u(z)-u\left(z^{\prime}\right)\right| \leq\left(\frac{C_{4}^{\prime}(n \gamma) \sqrt{\mathbf{e}\left(E, x_{0}, 25 r, e_{n}\right)+\Lambda r}}{r^{\gamma}}\right)\left|z-z^{\prime}\right|^{\gamma}
$$

what infers that $u \in C^{1, \gamma}\left(\mathbf{p} x_{0}+\mathbb{D}_{r}\right)$. From 4.21 and the Lipschitz Bound- 
ary Criterion (Theorem 3.1), we obtain

$$
\nu_{E}(y)= \pm \frac{(\nabla u(\mathbf{p} y),-1)}{\sqrt{1+|\nabla u(\mathbf{p} y)|^{2}}} \quad \forall y \in \mathbf{C}\left(x_{0}, r, e_{n}\right) \cap \partial E
$$

Since

$$
\begin{aligned}
F: \mathbb{R}^{n-1} & \longrightarrow \mathbb{R}^{n-1} \\
w & \longmapsto \frac{(-w, 1)}{\sqrt{1+|w|^{2}}}
\end{aligned}
$$

defines a Lipschitz function, if $x, y \in \mathbf{C}\left(x_{0}, r, e_{n}\right) \cap \partial E$, we immediately deduce that

$$
\begin{gathered}
\left|\nu_{E}(x)-\nu_{E}(y)\right| \leq \operatorname{Lip}(F)|\nabla u(\mathbf{p} x)-\nabla u(\mathbf{p} y)| \leq \\
\leq{ }^{4.28} \operatorname{Lip}(F)\left(\frac{C_{4}^{\prime}(n \gamma) \sqrt{\mathbf{e}\left(E, x_{0}, 25 r, e_{n}\right)+\Lambda r}}{r^{\gamma}}\right)|\mathbf{p} x-\mathbf{p} y|^{\gamma} \leq \\
\leq\left(\frac{C_{4}(n \gamma) \sqrt{\mathbf{e}\left(E, x_{0}, 25 r, e_{n}\right)+\Lambda r}}{r^{\gamma}}\right)|x-y|^{\gamma}
\end{gathered}
$$

where we have chosen $C_{4}(n, \gamma) \doteq \operatorname{Lip}(F) C_{4}^{\prime}(n, \gamma)$.

Proof of the Claim 1: We fix $x \in \mathbf{C}\left(x_{0}, r, e_{n}\right) \cap \partial E$, then $\mathbf{C}\left(x, 24 r, e_{n}\right) \subset$ C $\left(x_{0}, 25 r, e_{n}\right)$ implies

$$
\mathbf{e}\left(E, x, 24 r, e_{n}\right) \leq\left(\frac{25}{24}\right)^{n-1} \mathbf{e}\left(E, x_{0}, 25 r, e_{n}\right)
$$

Furthermore, by the definition of $\mathcal{E}$, we have

$$
\mathcal{E}_{x, e_{n}}(24 r) \leq\left(\frac{25}{24}\right)^{n-1} \mathcal{E}_{x_{0}, e_{n}}(25 r)
$$

Since $\alpha_{0}<1$ ensures $1<\frac{1}{\alpha_{0}^{n-1+2 \gamma}}$, we find that

$$
\begin{gathered}
\mathcal{E}_{x, e_{n}}(24 r) \leq \mathbf{e}\left(E, x, 24 r, e_{n}\right)+\frac{24 \Lambda r}{\alpha_{0}^{n-1+2 \gamma}} \leq \\
\leq \max \left\{\left(\frac{25}{24}\right)^{n-1}, \frac{24}{\alpha_{0}^{n-1+2 \gamma}}\right\}\left(\mathbf{e}\left(E, x_{0}, 25 r, e_{n}\right)+\Lambda r\right)
\end{gathered}
$$

In order to apply the Excess Improvement Theorem, we choose $\epsilon_{4}(n, \gamma) \leq$ $\max \left\{\left(\frac{25}{24}\right)^{n-1}, \frac{24}{\alpha_{0}^{n-1+2 \gamma}}\right\} \epsilon_{3}(n, \gamma)$, then, by 4.19 ,

$$
\mathcal{E}_{x, e_{n}}(24 r) \leq \epsilon_{3}(n, \gamma)
$$

what, from Theorem 4.4, provides the existence of a direction $\nu_{1}(x) \in$ $\mathbb{S}^{n-1}$ such that 4.14 and 4.15 holds true, namely

$$
\begin{gathered}
\mathcal{E}_{x, \nu_{1}(x)}\left(24 \alpha_{0} r\right) \leq \alpha_{0}^{2 \gamma} \mathcal{E}_{x, e_{n}}(24 r) \\
\left|\nu_{1}(x)-e_{n}\right|^{2} \leq C_{3}(n, \gamma) \mathcal{E}_{x, e_{n}}(24 r)
\end{gathered}
$$


Since $\alpha_{0}<1$, by 4.30 , we get that

$$
\mathcal{E}_{x, \nu_{1}(x)}\left(24 \alpha_{0} r\right) \leq \mathcal{E}_{x, e_{n}}(24 r) \leq \epsilon_{3}(n, \gamma)
$$

Note that $\mathbf{C}\left(x, 24 \alpha_{0} r, \nu_{1}(x)\right) \subset \mathbf{C}\left(x, 25 r, e_{n}\right)$ and thus $E$ is $\left(\Lambda, r_{0}\right)-$ minimizing in $\mathbf{C}\left(x, 24 \alpha_{0} r, \nu_{1}(x)\right)$. Therefore, 4.31 furnishes the conditions to apply the Excess Improvement at the smaller scale $24 \alpha_{0} r$ and direction $\nu_{1}(x)$. Iterating this process, we can show the existence of a sequence $\nu_{h} \doteq \nu_{h}(x) \in \mathbb{S}^{n-1}$ such that

$$
\begin{gathered}
\mathcal{E}_{x, \nu_{h}}\left(24 \alpha_{0}^{h} r\right) \leq \alpha_{0}^{2 \gamma h} \mathcal{E}_{x, e_{n}}(24 r) \\
\left|\nu_{h}-\nu_{h-1}\right|^{2} \leq C_{3}(n, \gamma) \mathcal{E}_{x, \nu_{h-1}}\left(24 \alpha_{0}^{h-1} r\right)
\end{gathered}
$$

The second inequality combined with 4.32 implies that

$$
\left|\nu_{h}-\nu_{h-1}\right|^{2} \leq C_{3}(n, \gamma) \alpha_{0}^{2 \gamma(h-1)} \mathcal{E}_{x, e_{n}}(24 r)
$$

If we set $\nu_{0}=e_{n}$, the inequalities 4.32 and 4.33 are valid for all $h \geq 1$. Let us prove the existence of the $\operatorname{limit} \lim _{h \rightarrow \infty} \nu_{h}$ by showing that $\left\{\nu_{h}\right\}_{h \in \mathbb{N}}$ is a Cauchy sequence. Indeed, by 4.33 , for all $j \geq h \geq 1$,

$$
\begin{aligned}
& \left|\nu_{j}-\nu_{h}\right| \leq \sum_{k=h}^{j}\left|\nu_{k}-\nu_{k-1}\right| \leq \sum_{k=h}^{j} \sqrt{C_{3}(n, \gamma) \alpha_{0}^{2 \gamma(h-1)} \mathcal{E}_{x, e_{n}}(24 r)} \leq \\
& \leq \sqrt{C_{3}(n, \gamma) \mathcal{E}_{x, e_{n}}(24 r)} \sum_{k=h}^{j} \alpha_{0}^{\gamma(h-1)}=\frac{\sqrt{C_{3}(n, \gamma) \mathcal{E}_{x, e_{n}}(24 r)}}{1-\alpha_{0}^{\gamma}} \alpha_{0}^{\gamma(h-1)}
\end{aligned}
$$

Thus, $\alpha_{0}<1$ implies that $\left\{\nu_{h}\right\}_{h \in \mathbb{N}}$ is a Cauchy sequence. Therefore, we can set $\nu(x)=\lim _{h \rightarrow \infty} \nu_{h}$. Since

$$
\left|\nu_{j}-\nu_{h}\right| \leq \frac{\sqrt{C_{3}(n, \gamma) \mathcal{E}_{x, e_{n}}(24 r)}}{1-\alpha_{0}^{\gamma}} \alpha_{0}^{\gamma(h-1)}
$$

we let $h=1$ and $j$ goes to $\infty$ and thus, by 4.29 , it becomes

$$
\left|\nu(x)-e_{n}\right|^{2} \leq \frac{25^{n-1} C_{3}(n, \gamma)}{24^{n-1}\left(1-\alpha_{0}^{\gamma}\right)} \mathcal{E}_{x, e_{n}}(25 r)
$$

for a suitable choice of the constant, that is exactly 4.25 . Let us turn to the proof of 4.24 , we take $s \in(0,12 r)$, since $\alpha_{0}^{0} 24 r=24 t$ and $\alpha_{0}^{h} 24 r \rightarrow$ 0 , we can find $h_{0} \geq 0$ such that $24 r \alpha_{0}^{h_{0}+1} \leq 2 s<24 r \alpha_{0}^{h_{0}}$. Evidently, $12 \sqrt{2} r<r_{0}$, then, from Proposition 2.9,

$$
\begin{array}{r}
\mathbf{e}(E, x, s, \nu(x)) \leq C_{d}(n)\left(\mathbf{e}\left(E, x, \sqrt{2} s, \nu_{h_{0}}\right)+\left|\nu(x)-\nu_{h_{0}}\right|^{2}\right) \leq \\
\leq^{2.5} C_{d}(n)\left(\left(\frac{24 r \alpha_{0}^{h_{0}}}{\sqrt{2} s}\right)^{n-1} \mathbf{e}\left(E, x, 24 r \alpha_{0}^{h_{0}}, \nu_{h_{0}}\right)+\left|\nu(x)-\nu_{h_{0}}\right|^{2}\right) \leq \\
\leq C_{d}^{\prime}(n)\left(\left(\frac{24 r \alpha_{0}^{h_{0}}}{s}\right)^{n-1} \mathcal{E}_{x, \nu_{h_{0}}}\left(24 r \alpha_{0}^{h_{0}}\right)+\left|\nu(x)-\nu_{h_{0}}\right|^{2}\right)
\end{array}
$$


By our choice of $h_{0}$, we have $\frac{24 r \alpha_{0}^{h_{0}}}{s} \leq \frac{2}{\alpha_{0}}$ and thus, by 4.32 ,

$$
\begin{array}{r}
\left(\frac{24 r \alpha_{0}^{h_{0}}}{s}\right)^{n-1} \mathcal{E}_{x, \nu_{h_{0}}}\left(24 r \alpha_{0}^{h_{0}}\right) \leq \frac{2^{n-1}}{\alpha_{0}^{n-1}} \alpha_{0}^{2 \gamma h_{0}} \mathcal{E}_{x, e_{n}}(24 r) \\
\leq \frac{2^{n-1}}{\alpha_{0}^{n-1}}\left(\frac{2 s}{24 r \alpha_{0}}\right)^{2 \gamma} \mathcal{E}_{x, e_{n}}(24 r) \leq \leq^{4.29} C_{4}(n, \gamma)\left(\frac{s}{r}\right)^{2 \gamma} \mathcal{E}_{x_{0}, e_{n}}(2)
\end{array}
$$

where we defined $C_{4}(n, \gamma)=C_{d}^{\prime}(n) \max \left\{\left(\frac{2^{2 \gamma} 25}{24^{2 \gamma} \alpha_{0}^{2 \gamma}}\right)^{n-1}, 1\right\}$. Combining 4.35 and the last inequality, we find that

$$
\mathbf{e}(E, x, s, \nu(x)) \leq C_{4}(n, \gamma)\left(\left(\frac{s}{r}\right)^{2 \gamma} \mathcal{E}_{x_{0}, e_{n}}(25 r)+\left|\nu(x)-\nu_{h_{0}}\right|^{2}\right)
$$

To control the second term on the right side of the last inequality, we let $j \rightarrow \infty$ in 4.34 and recall the choice of $h_{0}$, then

$$
\begin{aligned}
& \left|\nu(x)-\nu_{h_{0}}\right|^{2} \leq \frac{C_{3}(n, \gamma) \mathcal{E}_{x, e_{n}}(24 r)}{\left(1-\alpha_{0}^{\gamma}\right)^{2}} \alpha_{0}^{2 \gamma h_{0}} \leq \\
& \leq \frac{C_{3}(n, \gamma)}{\left(1-\alpha_{0}^{\gamma}\right)^{2}}\left(\frac{2 s}{24 r \alpha_{0}}\right)^{2 \gamma} \mathcal{E}_{x, e_{n}}(24 r) \leq \\
& \leq^{4.29} \overbrace{\frac{25^{n-1} C_{3}(n, \gamma) 2^{2 \gamma}}{\left(1-\alpha_{0}^{\gamma}\right)^{2} 24^{n-1+2 \gamma} \alpha_{0}^{2 \gamma}}}^{\doteq C_{4}^{\prime}(n, \gamma)}\left(\frac{s}{r}\right)^{2 \gamma} \mathcal{E}_{x_{0}, e_{n}}(25 r)
\end{aligned}
$$

Taking $C_{5}(n, \gamma)$ equal to the maximum between $C_{4}^{\prime}(n, \gamma)$ and $C_{4}(n, \gamma)$, by 4.36 and the last inequalities, it follows that

$$
\mathbf{e}(E, x, s, \nu(x)) \leq C_{5}(n, \gamma)\left(\frac{s}{r}\right)^{2 \gamma} \mathcal{E}_{x_{0}, e_{n}}(25 r) \quad \forall s \in(0,12 r)
$$

Since

$$
\frac{\Lambda s}{\alpha_{0}^{n-1+2 \gamma}} \leq \frac{25 \Lambda r}{\alpha_{0}^{n-1+2 \gamma}}
$$

by the last inequality, the definition of $\mathcal{E}$ and 4.37 , we conclude that

$$
\mathcal{E}_{x, \nu(x)}(s) \leq C_{5}(n, \gamma)\left(\frac{s}{r}\right)^{2 \gamma} \mathcal{E}_{x_{0}, e_{n}}(25 r) \quad \forall s \in(0,12 r)
$$

Finally, we will prove 4.26 . To this end, we note that 4.38 holds true for $24 r$ in place of $s$ and thus it suffices to prove

$$
\mathbf{e}\left(E, x, s, e_{n}\right) \leq C_{3}^{\prime}(n, \gamma) \mathbf{e}\left(E, x_{0}, e_{n}, 25 r\right) \quad \forall s \in(0,24 r)
$$

Firstly, suppose that $s \in[6 r, 24 r)$, then $\frac{r}{s} \leq \frac{1}{6}$ and $\mathbf{C}\left(x, s, e_{n}\right) \subset \mathbf{C}\left(x_{0}, 25 r, e_{n}\right)$ ensure that

$$
\mathbf{e}\left(E, x, s, e_{n}\right) \leq\left(\frac{25 r}{s}\right)^{n-1} \mathbf{e}\left(E, x_{0}, 25 r, e_{n}\right) \leq\left(\frac{25}{6}\right)^{n-1} \mathbf{e}\left(E, x_{0}, 25 r, e_{n}\right)
$$


If, otherwise, $s \in(0,6 r)$, by Proposition 2.9, we have that

$$
\mathbf{e}\left(E, x, s, e_{n}\right) \leq C_{d}(n)\left(\mathbf{e}(E, x, \sqrt{2} s, \nu(x))+\left|\nu(x)-e_{n}\right|^{2}\right)
$$

We note that $\sqrt{2} s<12 r$. Therefore, we can take into account 4.24 and 4.25. Then

$$
\mathbf{e}\left(E, x, s, e_{n}\right) \leq C_{d}(n) C_{3}^{\prime}(n, \gamma)\left(\left(\frac{\sqrt{2} s}{r}\right)^{2 \gamma} \mathcal{E}_{x_{0}, e_{n}}(25 r)+\mathcal{E}_{x_{0}, e_{n}}(25 r)\right)
$$

In order to finish the proof of the Claim, note that $s \in(0,6 r)$ implies $\frac{s}{r}<6$.

Proof of the Claim 2: We fix $s \in(0, r)$ and $z \in\left(\mathbf{p} x_{0}+\mathbb{D}_{r}\right)$, by 4.25 , up to decreasing $\epsilon_{4}(n, \gamma)$, we can henceforth assume that

$$
\frac{1}{\sqrt{2}} \leq \mathbf{q} \nu(x) \quad \forall x \in \mathbf{C}\left(x_{0}, r, e_{n}\right) \cap \partial E
$$

Therefore, it is straightforward to conclude that

$$
\mathbf{C}\left(x, s, e_{n}\right) \subset \mathbf{B}(x, \sqrt{2} s) \subset \mathbf{C}(x, 2 s, \nu(x))
$$

Thus, by the set inclusions above and the definition of excess, we can affirm that

$$
\int_{\mathbf{C}\left(x, s, e_{n}\right) \cap \partial^{*} E} \frac{\left|\nu_{E}-\nu(x)\right|^{2}}{2} \mathrm{~d} \mathcal{H}_{n-1} \leq(2 s)^{n-1} \mathbf{e}(E, x, 2 s, \nu(x))
$$

whenever $x \in \mathbf{C}\left(x_{0}, r, e_{n}\right) \cap \partial E$. From 4.40 , for any $x \in \mathbf{C}\left(x_{0}, r, e_{n}\right) \cap \partial E$ we have $\mathbf{q} \nu(x)>0$. Then, we can define

$$
\begin{aligned}
X: \mathbf{C}\left(x_{0}, r, e_{n}\right) \cap \partial E & \longrightarrow \mathbb{R}^{n-1} \\
x & \longmapsto-\frac{\mathbf{p} \nu(x)}{\mathbf{q} \nu(x)}
\end{aligned}
$$

We note that

$$
\sqrt{1+|X(x)|^{2}}=\frac{\sqrt{|\mathbf{p} \nu(x)|^{2}+|\mathbf{q} \nu(x)|^{2}}}{|\mathbf{q} \nu(x)|}={ }^{\nu(x) \in \mathbb{S}^{n-1}} \frac{1}{|\mathbf{q} \nu(x)|}
$$

Thus, we immediately deduce that

$$
\begin{array}{r}
|X(x)| \leq 1 \\
\mathbf{p} \nu(x)=-\frac{X(x)}{\sqrt{1+|X(x)|^{2}}} \\
\mathbf{q} \nu(x)=\frac{1}{\sqrt{1+|X(x)|^{2}}}
\end{array}
$$

To infer that $|X(x)| \leq 1$, we required the help of 4.40 and $1=|\nu(x)|^{2}=$ $|\mathbf{p} \nu(x)|^{2}+|\mathbf{q} \nu(x)|^{2}$. Henceforth, we assume that 4.23 holds true, if not, 
it suffices to work with $-X$ in place of $X$. Since $y \in \mathbf{C}\left(x_{0}, r, e_{n}\right) \cap \partial E$ implies $y=(\mathbf{p} y, \mathbf{q} y) \in \mathbf{C}\left(x_{0}, r, e_{n}\right) \cap\left(x_{0}+G(u)\right)$, the Lipschitz Boundary Criterion (Theorem 3.1) ensures that

$$
\nu_{E}(y)=\nu_{E}(\mathbf{p} y, u(\mathbf{q} y))=-\frac{(\nabla u(\mathbf{p} y),-1)}{\sqrt{1+\mid \nabla u(\mathbf{p} y)^{2}}}
$$

and thus, by 4.42 , we find that

$$
\begin{gathered}
\int_{\mathbf{C}\left(x, s, e_{n}\right) \cap \partial^{*} E} \frac{\left|\nu_{E}(y)-\nu(x)\right|^{2}}{2} \mathrm{~d} \mathcal{H}_{n-1}(y)= \\
\frac{1}{2} \int_{\mathbf{C}\left(x, s, e_{n}\right) \cap \partial^{*} E}\left|-\frac{\nabla u(\mathbf{p} y)}{\sqrt{1+|\nabla u(\mathbf{p} y)|^{2}}}+\frac{X(x)}{\sqrt{1+|X(x)|^{2}}}\right|^{2} \mathrm{~d} \mathcal{H}_{n-1}(y)+ \\
+\frac{1}{2} \int_{\mathbf{C}\left(x, s, e_{n}\right) \cap \partial^{*} E}\left|\frac{1}{\sqrt{1+\mid \nabla u(\mathbf{p} y)^{2}}}-\frac{1}{\sqrt{1+|X(x)|^{2}}}\right|^{2} \mathrm{~d} \mathcal{H}_{n-1}(y)
\end{gathered}
$$

From 4.21, we obtain that $x=(z, u(z)) \in \partial E \cap \mathbf{C}\left(x_{0}, r, e_{n}\right)$. By Theorem 9.1 in [Mag12] and the last inequalities, we deduce that

$$
\begin{array}{r}
\int_{\mathbf{C}\left(x, s, e_{n}\right) \cap \partial^{*} E} \frac{\left|\nu_{E}(y)-\nu(x)\right|^{2}}{2} \mathrm{~d} \mathcal{H}_{n-1} y= \\
=\frac{1}{2} \int_{z+\mathbb{D}_{s}}\left|-\frac{\nabla u(w)}{\sqrt{1+|\nabla u(w)|^{2}}}+\frac{X(x)}{\sqrt{1+|X(x)|^{2}}}\right|^{2} \sqrt{1+|\nabla u(w)|^{2}} \mathrm{~d} \mathcal{H}_{n-1}(w)+ \\
+\frac{1}{2} \int_{z+\mathbb{D}_{s}}\left|\frac{1}{\sqrt{1+|\nabla u(w)|^{2}}}-\frac{1}{\sqrt{1+|X(x)|^{2}}}\right|^{2} \sqrt{1+|\nabla u(w)|^{2}} \mathrm{~d} \mathcal{H}_{n-1}(w)
\end{array}
$$

We claim that

$$
\int_{z+\mathbb{D}_{s}}\left|\nabla u-(\nabla u)_{z, s}\right|^{2}=\min _{v \in \mathbb{R}^{n}} \int_{z+\mathbb{D}_{s}}|\nabla u-v|^{2}
$$

Since $|\nabla u|^{2}$ and $|X(x)|^{2}$ both are less or equal than 1 , by 4.44 , we have that

$$
\begin{gathered}
\int_{z+\mathbb{D}_{s}}\left|\nabla u-(\nabla u)_{z, s}\right|^{2} \leq \int_{z+\mathbb{D}_{s}}|\nabla u-X(x)|^{2} \leq \\
\leq \int_{z+\mathbb{D}_{s}}\left|\frac{\nabla u-X(x)}{\sqrt{1+|X(x)|^{2}}}\right|^{2} \sqrt{1+|\nabla u|^{2}} \leq \\
\int_{z+\mathbb{D}_{s}} \mid \frac{\nabla u}{\sqrt{1+|X(x)|^{2}}}-\frac{\nabla u}{\sqrt{1+|\nabla u|^{2}}}+\frac{\nabla u}{\sqrt{1+|\nabla u|^{2}}-\left.\frac{X(x)}{\sqrt{1+|X(x)|^{2}}}\right|^{2} \sqrt{1+|\nabla u|^{2}}} \\
\leq \int_{z+\mathbb{D}_{s}}\left|\frac{1}{\sqrt{1+|X(x)|^{2}}}-\frac{1}{\sqrt{1+|\nabla u|^{2}}}\right|^{2} \sqrt{1+|\nabla u|^{2}}+ \\
+\int_{z+\mathbb{D}_{s}}\left|-\frac{\nabla u}{\sqrt{1+|\nabla u|^{2}}}+\frac{X(x)}{\sqrt{1+|X(x)|^{2}}}\right|^{2} \sqrt{1+|\nabla u|^{2}}
\end{gathered}
$$


Therefore, by 4.43 , we obtain that

$$
\int_{z+\mathbb{D}_{s}}\left|\nabla u-(\nabla u)_{z, s}\right|^{2} \leq 2 \int_{\mathbf{C}\left(x, s, e_{n}\right) \cap \partial^{*} E} \frac{\left|\nu_{E}(y)-\nu(x)\right|^{2}}{2} \mathrm{~d} \mathcal{H}_{n-1} y
$$

Then, by 4.24, 4.41 and the last inequality, we finally obtain that

$$
\begin{aligned}
& \frac{1}{s^{n-1}} \int_{z+\mathbb{D}_{s}}\left|\nabla u-(\nabla u)_{z, s}\right|^{2} \leq 2^{n} \mathbf{e}(E, x, 2 s, \nu(x)) \leq \\
& \leq 2^{n} \mathcal{E}_{x, \nu(x)}(2 s) \leq 2^{n-1} C_{3}^{\prime}(n, \gamma)\left(\frac{s}{r}\right)^{2 \gamma} \mathcal{E}_{x_{0}, e_{n}}(25 r)
\end{aligned}
$$

for all $s \in(0,6 r)$, what concludes the proof of the Claim 2 (4.27). In order to prove 4.44 , we note that $F(v)=\int_{z+\mathbb{D}_{s}}|\nabla u-v|^{2}, \forall v \in \mathbb{R}^{n-1}$ is a differentiable function and it is straightforward to calculate that

$$
\nabla F(v)=-2 \int_{z+\mathbb{D}_{s}} \nabla u-v=2\left|z+\mathbb{D}_{s}\right| v-2 \int_{z+\mathbb{D}_{s}} \nabla u
$$

what implies that $\nabla F(v)=0$ if, and only if, $v=(\nabla u)_{z, s}$. Then $(\nabla u)_{z, s}$ is the unique critical point of $F$. Since $F$ is a convex function, we conclude that $(\nabla u)_{z, s}$ is the minimum point of $F$ which ensures 4.44 . 


\section{Bibliography}

[All72] William K. Allard. On the first variation of a varifold. Ann. of Math. (2), 95:417-491, 1972. 2

[All87] William K. Allard. Notes on the theory of varifolds. Astérisque, (154-155):8, 73-93, 349-350 (1988), 1987. Théorie des variétés minimales et applications (Palaiseau, 1983-1984). 2

[ALM65] F. ALMGREN. The theory of varifolds. 1965. 2

[Alm76] Almgren. Existence and regularity almost everywhere of solutions to elliptic variational problems with constraints. 1976. 2

[(au09] Gerd Grubb (auth.). Distributions and Operators. Graduate Texts in Mathematics 252. Springer-Verlag New York, 1 edition, 2009. 35, 48

[Cac27] Renato Caccioppoli. Sulla quadratura delle superfici piane e curve. page pp. 142?146, VI (1927). 1

[DG54] Ennio De Giorgi. Su una teoria generale della misura $(r-1)$ dimensionale in uno spazio ad $r$ dimensioni. Ann. Mat. Pura Appl. (4), 36:191-213, 1954. 1

[DG55] Ennio De Giorgi. Nuovi teoremi relativi alle misure $(r-1)$ dimensionali in uno spazio ad $r$ dimensioni. Ricerche Mat., 4:95-113, 1955. 1

[DG61a] Ennio De Giorgi. Complementi alla teoria della misura $(n-$ 1)-dimensionale in uno spazio n-dimensionale. Seminario di Matematica della Scuola Normale Superiore di Pisa, 1960-61. Editrice Tecnico Scientifica, Pisa, 1961. 1

[DG61b] Ennio De Giorgi. Frontiere orientate di misura minima. Seminario di Matematica della Scuola Normale Superiore di Pisa, 1960-61. Editrice Tecnico Scientifica, Pisa, 1961. 1, 2

[Fed96] Herbert Federer. Geometric measure theory. Classics in mathematics. Springer, 1996. 1, 2

[FF60] Herbert Federer and Wendell H. Fleming. Normal and integral currents. Ann. of Math. (2), 72:458-520, 1960. 1 
[FL03] Xiaoping Yang Fanghua Lin. Geometric Measure Theory - An Introduction, volume Vol 1 of Advanced Mathematics. International Press, 2003. 1, 22, 23

[Fol95] Gerald B. Folland. Introduction to partial differential equations. Princeton University Press, 2 sub edition, 1995. 59, 60

[Fol99] Gerald B. Folland. Real analysis: modern techniques and their applications. PAM. Wiley, 2 edition, 1999. 16, 33, 39, 40, 42, $60,62,69$

[Giu84] Enrico Giusti. Minimal Surfaces and Functions of Bounded Variation. Monographs in Mathematics. Birkhäuser Boston, 1 edition, 1984. 1, 2, 9, 11, 28

[Gor94] Russell A. Gordon. The integrals of Lebesgue, Denjoy, Perron, and Henstock. Graduate Studies in Mathematics 004. American Mathematical Society, 1994. 17

[J.05] Heinonen J. Lectures on Lipschitz analysis. draft, 2005. 52

[LA00] Diego Pallara Luigi Ambrosio, Nicola Fusco. Functions of bounded variation and free discontinuity problems. Oxford Mathematical Monographs. Oxford University Press, USA, 2000. 1, 2, 14, 44, 49, 51, 74

[LCE92] Ronald F. Gariepy Lawrence Craig Evans. Measure theory and fine properties of functions. Studies in advanced mathematics. CRC Press, 1 edition, 1992. 1, 6, 10, 15, 17, 21, 24, 25, 26, 30, $32,39,49,52,55,56,61$

[Mag12] Francesco Maggi. Sets of Finite Perimeter and Geometric Variational Problems: An Introduction to Geometric Measure Theory. Cambridge Studies in Advanced Mathematics 135. Cambridge University Press, 1st edition, 2012. i, 1, 2, 3, 8, 11, 15, $16,17,18,19,21,23,28,35,36,37,44,46,49,54,55,56,57$, $58,64,79$

[Mas75] Umberto Massari. Frontiere orientate di curvatura media assegnata in $l^{p}$. Rendiconti del Seminario Matematico della Università di Padova, 53:37-52, 1975. 2, 3

[Mor00] Frank Morgan. Geometric measure theory: a beginner's guide. Academic Press, 3 edition, 2000. 1

[Rud91] Walter Rudin. Functional Analysis. International series in pure and applied mathematics. McGraw-Hill, 2nd ed edition, 1991. 62

[Sim83] Leon Simon. Lectures on geometric measure theory. Proceedings of the Centre for Mathematical Analysis, Australian National University 3. Centre for Mathematical Analysis, Australian National University, 1 edition, 1983. 1, 2, 54 
[Tam84] Italo Tamaninni. Regularity results for almost minimal oriented hypersurfaces in $R N$. Quaderni di Matematica, 1, 1 edition, 1984. 2,3

[VOL] https://alexandervolkmann.files.wordpress.com/2014/10/diplom.pdf. 13,30 